QUARTERLY OF APPLIED MATHEMATICS

VOLUME LXVI, NUMBER 4

DECEMBER 2008, PAGES 659-705

S $0033-569 X(08) 01087-X$

Article electronically published on October 1, 2008

\title{
THE INTERFACE COUPLING OF THE GAS DYNAMICS EQUATIONS
}

\author{
BY \\ CHRISTOPHE CHALONS (UMR 7598 Laboratoire Jacques-Louis Lions, Université Paris 7-Denis \\ Diderot, Boîte courrier 187, 75252 Paris Cedex 05, France), \\ PIERRE-ARNAUD RAVIART (UMR 7598 Laboratoire Jacques-Louis Lions, CNRS 8 Université \\ Pierre et Marie Curie-Paris 6, Bô̂te courrier 187, 75252 Paris Cedex 05, France), \\ AND \\ NICOLAS SEGUIN (UMR 7598 Laboratoire Jacques-Louis Lions, Université Pierre et Marie \\ Curie-Paris 6, Bô̂te courrier 187, 75252 Paris Cedex 05, France)
}

\begin{abstract}
We investigate the one-dimensional coupling of two systems of gas dynamics at a fixed interface. The coupling constraints consist in requiring the continuity of a system of nonconservative variables at the interface. Since we are dealing with hyperbolic systems, weak coupling conditions are proposed. The existence and the uniqueness of the solutions of the coupled Riemann problem are investigated. Several examples of solutions satisfying the weak coupling conditions are contructed, either continuous or discontinuous with respect to the nonconservative variables at the interface.
\end{abstract}

Introduction. The problem of coupling various mathematical models described by systems of partial differential equations is a topic of increasing importance. It arises for instance in the modelling of complex industrial devices in engineering. The device is divided into different parts which are modelled using different approximations. It then remains to couple these models at the interfaces separating the various parts of the device for obtaining a global mathematical model. As a constraint, the coupling procedures have to be as local as possible in order to be easily implemented numerically.

Such coupling problems are encountered in several applications. First, they naturally arise in thermohydraulics in connection with the modelling of the primary coolant circuit of a pressurized water reactor in a nuclear power plant. Various multiphase fluid models are used involving systems of partial differential equations having a variable number of equations supplemented with various closure relations. Similar situations appear also

Received March 16, 2007.

2000 Mathematics Subject Classification. Primary 76N15, 35F25, 35L65.

E-mail address: chalons@math.jussieu.fr

E-mail address: pa@raviart.com

E-mail address: seguin@ann.jussieu.fr

(C)2008 Brown University 
in the modelling of two-fluid (or multi-fluid) flows, where the dynamics is provided by one of the fluids. The two fluids possess their own equation of state and are coupled at a moving interface. See AK01 and the extensive literature on this subject. We can also mention the coupling problems arising in networks CG06, BHK06a, BHK06b] and traffic flows [GP06a, GP06b, HR06] which have deserved a growing interest in the last few years.

In this paper, we are indeed concerned with applications to nuclear reactors 1 The overall coupling problem in this context is a very difficult and challenging one at least from the mathematical point of view and must be decomposed into elementary though already complicated subproblems. Here we focus our attention on the coupling of two Euler systems of compressible gas dynamics provided with different equations of state. This is indeed a first step in the mathematical analysis of the coupling of two thermohydraulic models. Our approach follows that initiated in GR04 in the scalar case and in GLTR05] in the case of systems encountered in plasma physics. In this paper, we study a fairly general approach of the coupling problem, referred to as the state coupling method. This coupling method has been investigated in the framework of Lagrangian systems in $\mathrm{ACC}^{+} 06 \mathrm{~b}, \mathrm{ACC}^{+} 06 \mathrm{c}$, and numerical state coupling methods have been proposed in $\mathrm{ACC}^{+} 05 \mathrm{a}, \mathrm{ACC}^{+} 05 \mathrm{~b}, \mathrm{ACC}^{+} 06 \mathrm{a}$. At the difference of the flux coupling method (see $\mathrm{ACC}^{+}$05a, $\mathrm{ACC}^{+} 06 \mathrm{a}, \mathrm{Hur06}$ and [T92, KR95, BV06]) where one imposes the continuity of the flux at the interface, we impose here the continuity (in a weak sense) at the interface of a set $\mathbf{V}$ of variables which are not necessarily conservative. This method appears to be very flexible in the sense that we can ensure the global conservation of mass and momentum or energy for the coupled problem depending on the proper choice of the set $\mathbf{V}$. In practice, the choice of the information $\mathbf{V}$ that must be transmitted at the interface is given at the level of the physical modelling and generally depends on the coupling problem. Now, a natural mathematical question is to solve the coupled Riemann problem. This paper is a contribution to the solution of this problem, which appears far more complicated that one could think a priori. It is worth noting that a different approach could be followed, introducing an appropriate Dirac measure at the interface in a global model in order to recover the continuity of $\mathbf{V}$ at the interface, as was done in the scalar case by Boutin [Bou06] (see also [IT95, GL96, GL04]).

The plan of this paper is as follows. In Section 1, we introduce the general state coupling method. In the next two sections, we establish preliminary results. Section 2 is devoted to the analysis of the signs of the speeds of gas dynamics waves. Then, in Section 3 , we characterize the set of states $\mathbf{U}$ that can be connected to a given state $\mathbf{U}_{0}$ on the left (resp. on the right) by a sequence of a 1 -wave, a 2 -wave and a 3 -wave whose speeds are nonnegative (resp. nonpositive). Provided with the tools developed in the previous sections, we are able in Section 4 to characterize the forms of all possible solutions to the coupled Riemann problem which are $\mathbf{V}$-continuous at the interface $x=0$. We prove the existence and uniqueness of subsonic solutions. We then consider solutions which may be $\mathbf{V}$-discontinuous at the interface. Such solutions need to satisfy at $x=0$ coupling

\footnotetext{
${ }^{1}$ The authors of the present paper are involved in a joint research program on multiphase flows between $\mathrm{CEA}$ and Université Pierre et Marie Curie-Paris 6 (see $\mathrm{ACC}^{+} 05 \mathrm{a}, \mathrm{ACC}^{+} 05 \mathrm{~b}, \mathrm{ACC}^{+} 06 \mathrm{~b}$ $\mathrm{ACC}^{+} 06 \mathrm{a}, \mathrm{ACC}^{+} 06 \mathrm{c}$ and the references therein) in the framework of the Neptune project.
} 
constraints that we characterize geometrically in Section 5. In Section 6, we determine the forms of all possible $\mathbf{V}$-discontinuous solutions. As an application, we consider the coupled Riemann problem whose left and right initial velocities are equal: this is the case for instance for a matter-wave which crosses the interface. In Section 7, we solve this problem for "almost" all such initial data and we give the form of each solution which can be easily computed. We thus obtain a number of explicit exact solutions that can serve as benchmarks for numerical methods of solution of the coupled Cauchy problem.

1. The problem of coupling two gas dynamics systems at a fixed interface. We want to couple two gas dynamics systems corresponding to two different equations of state at a fixed interface located at $x=0$. This amounts to solving the systems

$$
\begin{aligned}
& \partial_{t} \mathbf{U}+\partial_{t} \mathbf{F}_{L}(\mathbf{U})=\mathbf{0}, x<0, t>0, \\
& \partial_{t} \mathbf{U}+\partial_{t} \mathbf{F}_{R}(\mathbf{U})=\mathbf{0}, x>0, t>0,
\end{aligned}
$$

where for $\alpha=L, R$,

$$
\mathbf{U}=\left(\begin{array}{c}
\rho \\
\rho u \\
\rho e
\end{array}\right), \mathbf{F}_{\alpha}(\mathbf{U})=\left(\begin{array}{c}
\rho u \\
\rho u^{2}+p_{\alpha} \\
\left(\rho e+p_{\alpha}\right) u
\end{array}\right)
$$

and $p_{\alpha}=p_{\alpha}(\rho, \varepsilon)$ may be given for instance as a function of $\rho$ and $\varepsilon$. These systems are supplemented by an initial condition

$$
\mathbf{U}(x, 0)=\mathbf{U}_{0}(x)
$$

and coupling conditions that we shall define below. Recall that $\rho$ denotes the density of the fluid, $u$ its velocity, $\varepsilon$ its specific internal energy and $e=\varepsilon+u^{2} / 2$ its specific total energy. The pressure $p$ of the fluid is thus defined by

$$
p= \begin{cases}p_{L}(\rho, \varepsilon), & \text { if } x<0 \\ p_{R}(\rho, \varepsilon), & \text { if } x>0 .\end{cases}
$$

We denote by $\Omega$ the state space

$$
\Omega=\left\{\mathbf{U} \in \mathbb{R}^{3} ; \rho>0, p>0\right\} .
$$

Concerning the coupling conditions at $x=0$, we want to ensure "as far as possible" the continuity of some system $\mathbf{V}$ of variables, i.e., we would like to impose the following constraint on the traces of $\mathbf{V}(., t)$ at $x=0$ :

$$
\mathbf{V}\left(0_{+}, t\right)=\mathbf{V}\left(0_{-}, t\right) .
$$

More precisely, we are given two $\mathbf{C}^{1}$ diffeomorphisms $\Theta_{\alpha}: \mathbf{V} \in \Omega_{V} \mapsto \mathbf{U}=\Theta_{\alpha}(\mathbf{V}) \in$ $\Omega, \alpha=L, R$ and we would like to impose

$$
\Theta_{L}^{-1}\left(\mathbf{U}\left(0_{-}, t\right)\right)=\Theta_{R}^{-1}\left(\mathbf{U}\left(0_{+}, t\right)\right) .
$$

At least three choices of $\mathbf{V}$ are of practical interest:

(i) $\mathbf{V}=\mathbf{U}, \Theta_{\alpha}=I d$; 
(ii) $\mathbf{V}=\left(\begin{array}{c}\rho \\ u \\ p\end{array}\right), \Theta_{\alpha}(\mathbf{V})=\left(\begin{array}{c}\rho \\ \rho u \\ \rho e_{\alpha}\end{array}\right), e_{\alpha}=\varepsilon_{\alpha}(\rho, p)+u^{2} / 2$,

where $p \mapsto \varepsilon_{\alpha}(\rho, p)$ is the inverse function of $\varepsilon \mapsto p_{\alpha}(\rho, \varepsilon)$, i.e.,

(iii) $\mathbf{V}=\left(\begin{array}{c}\rho \\ u \\ h\end{array}\right), \Theta_{\alpha}(\mathbf{V})=\left(\begin{array}{c}\rho \\ \rho u \\ \rho e_{\alpha}\end{array}\right), \rho e_{\alpha}=\rho h-p_{\alpha}(\rho, h)+\rho u^{2} / 2$,

where $h=\varepsilon+p / \rho$ denotes the specific enthalpy and the equations of state are taken in the form

$$
p=p_{\alpha}(\rho, h), \alpha=L, R .
$$

Indeed, suppose that we ensure the exact continuity of $\mathbf{V}$ at the interface. Then, the choice (i) guarantees the global mass conservation but not the global momentum and energy conservations since only the first component $\rho u$ of the flux function is continuous at the interface. On the other hand, the choice (ii) guarantees the continuity of $\rho u$ and $\rho u^{2}+p$ but not that of $(\rho e+p) u$ at the interface so that here the global mass and momentum conservations are preserved but not the global energy conservation. Alternatively, the choice (iii) guarantees the continuity of $\rho u$ and $\rho h u=(\rho e+p) u$ at the interface but not that of $\rho u^{2}+p$, which implies that the global mass and energy conservations are preserved but not the global momentum conservation.

Returning to the general case, we note that the continuity condition (5) is too restrictive and cannot be satisfied at least in general. This is easily seen by considering the geometry of characteristics of both hyperbolic systems (11) and (2) at the interface $x=0$. As in GR04, GLTR05, we weaken this condition in the following way. We denote by $\mathbf{W}_{\alpha}\left(x / t ; \mathbf{U}_{G}, \mathbf{U}_{D}\right)$ the solution of the Riemann problem

$$
\left\{\begin{array}{l}
\partial_{t} \mathbf{U}+\partial_{x} \mathbf{F}_{\alpha}(\mathbf{U})=\mathbf{0}, x \in \mathbb{R}, t>0, \\
\mathbf{U}(x, 0)= \begin{cases}\mathbf{U}_{G}, & \text { if } x<0 \\
\mathbf{U}_{D}, & \text { if } x>0\end{cases}
\end{array}\right.
$$

and by $\mathbf{Z}_{\alpha}\left(x / t ; \mathbf{V}_{G}, \mathbf{V}_{D}\right)$ the solution of this Riemann problem expressed in the variables $\mathbf{V}$, i.e.,

$$
\mathbf{Z}_{\alpha}\left(x / t ; \mathbf{V}_{G}, \mathbf{V}_{D}\right)=\Theta_{\alpha}^{-1}\left(\mathbf{W}_{\alpha}\left(x / t ; \Theta_{\alpha}\left(\mathbf{V}_{G}\right), \Theta_{\alpha}\left(\mathbf{V}_{D}\right)\right)\right) .
$$

Next, we define the sets

$$
\mathcal{O}_{L}\left(\mathbf{V}_{D}\right)=\left\{\mathbf{Z}_{L}\left(0_{-} ; \mathbf{V}, \mathbf{V}_{D}\right) ; \mathbf{V} \in \Omega_{V}\right\}
$$

and

$$
\mathcal{O}_{R}\left(\mathbf{V}_{G}\right)=\left\{\mathbf{Z}_{R}\left(0_{+} ; \mathbf{V}_{G}, \mathbf{V}\right) ; \mathbf{V} \in \Omega_{V}\right\}
$$

Note that

$$
\mathcal{O}_{L}\left(\mathbf{V}_{D}\right)=\Theta_{L}^{-1}\left(\left\{\mathbf{W}_{L}\left(0_{-} ; \mathbf{U}, \Theta_{L}\left(\mathbf{V}_{D}\right)\right) ; \mathbf{U} \in \Omega\right\}\right)
$$

and

$$
\mathcal{O}_{R}\left(\mathbf{V}_{G}\right)=\Theta_{R}^{-1}\left(\left\{\mathbf{W}_{R}\left(0_{+} ; \Theta_{R}\left(\mathbf{V}_{G}\right), \mathbf{U}\right) ; \mathbf{U} \in \Omega\right\}\right)
$$


Then, the coupling conditions at $x=0$, which have to be viewed as a weak form of (5), read:

$$
\begin{aligned}
\mathbf{V}\left(0_{-}, t\right) & \in \mathcal{O}_{L}\left(\mathbf{V}\left(0_{+}, t\right)\right), \\
\mathbf{V}\left(0_{+}, t\right) & \in \mathcal{O}_{R}\left(\mathbf{V}\left(0_{-}, t\right)\right),
\end{aligned}
$$

or equivalently

$$
\left\{\begin{array}{l}
\mathbf{U}\left(0_{-}, t\right) \in\left\{W_{L}\left(0_{-} ; \mathbf{U}, \Theta_{L}\left(\Theta_{R}^{-1}\left(\mathbf{U}\left(0_{+}, t\right)\right)\right)\right) ; \mathbf{U} \in \Omega\right\} \\
\mathbf{U}\left(0_{+}, t\right) \in\left\{W_{R}\left(0_{+} ; \Theta_{R}\left(\Theta_{L}^{-1}\left(\mathbf{U}\left(0_{-}, t\right)\right), \mathbf{U}\right)\right) ; \mathbf{U} \in \Omega\right\} .
\end{array}\right.
$$

This method of coupling will be referred to in the sequel as the $\boldsymbol{V}$-state coupling method.

Now, a fundamental question from both the theoretical and numerical points of view is the study of the $\mathbf{V}$-coupled Riemann problem, which consists in solving Eqs.(11), (2) with the coupling conditions (10), (111) and the initial condition

$$
\mathbf{U}_{0}(x)= \begin{cases}\mathbf{U}_{G}, & \text { if } x<0, \\ \mathbf{U}_{D}, & \text { if } x>0 .\end{cases}
$$

2. Analysis of the wave speeds. As a preliminary step for the solution of the coupled Riemann problem, we need to study the sign of the speed of the gas dynamics waves. We begin by making precise some notation that we will use in all of the sequel and by recalling some standard results concerning these waves. Let us first characterize the states $\mathbf{U}$ that can be connected to a given state $\mathbf{U}_{0}$ by an admissible (i.e., entropic) shock wave. We then assume the following simplifying hypothesis:

H.1. There exists a unique function $p \mapsto \tau=h_{0}(p)$ that is a solution of the Hugoniot equation

$$
\varepsilon(\tau, p)-\varepsilon\left(\tau_{0}, p_{0}\right)+\frac{1}{2}\left(p+p_{0}\right)\left(\tau-\tau_{0}\right)=0, \tau=\frac{1}{\rho} .
$$

Moreover, this function is monotonically decreasing, strictly convex and satisfies $h_{0}\left(p_{0}\right)=$ $\tau_{0}$.

Then the states $\mathbf{U}$ that can be connected to $\mathbf{U}_{0}$ on the right by an admissible 1-shock wave are given by

$$
u=u_{0}-\sqrt{\left(p-p_{0}\right)\left(\tau_{0}-h_{0}(p)\right)}, \rho=\frac{1}{h_{0}(p)}, p>p_{0},
$$

while the states $\mathbf{U}$ that can be connected to $\mathbf{U}_{0}$ on the left by an admissible 3 -shock wave are characterized by

$$
u=u_{0}+\sqrt{\left(p-p_{0}\right)\left(\tau_{0}-h_{0}(p)\right)}, \rho=\frac{1}{h_{0}(p)}, p<p_{0} .
$$

We next look for the states $\mathbf{U}$ that can be connected to $\mathbf{U}_{0}$ by a rarefaction wave. For convenience, we take the equation of state in the form

$$
\rho=\rho(p, s)
$$

so that the sound speed is given by

$$
c=c(p, s)=\left(\partial_{p} \rho\right)^{-\frac{1}{2}} .
$$


Then, the states $\mathbf{U}$ that can be connected to $\mathbf{U}_{0}$ on the right by a 1-rarefaction wave are characterized by

$$
u=u_{0}-\int_{p_{0}}^{p} \frac{d p}{(\rho c)\left(p, s_{0}\right)}, \rho=\rho\left(p, s_{0}\right), p<p_{0}
$$

while the states $\mathbf{U}$ that can be connected to $\mathbf{U}_{0}$ on the left by a 3-rarefaction wave are given by

$$
u=u_{0}+\int_{p_{0}}^{p} \frac{d p}{(\rho c)\left(p, s_{0}\right)}, \rho=\rho\left(p, s_{0}\right), p>p_{0} .
$$

If we set

$$
\Phi_{0}(p)= \begin{cases}\int_{p_{0}}^{p} \frac{d p}{(\rho c)\left(p, s_{0}\right)}, & \text { if } p \leq p_{0}, \\ \sqrt{\left(p-p_{0}\right)\left(\tau_{0}-h_{0}(p)\right)}, & \text { if } p \geq p_{0},\end{cases}
$$

and

$$
\varphi_{0}(p)= \begin{cases}\rho\left(p, s_{0}\right), & \text { if } p \leq p_{0} \\ \frac{1}{h_{0}(p)}, & \text { if } p \geq p_{0}\end{cases}
$$

then we obtain that the 1 -wave curve $\mathcal{C}^{1}\left(\mathbf{U}_{0}\right)$ of states $\mathbf{U}$ which can be connected on the right by an admissible 1-wave is given by

$$
u=u_{0}-\Phi_{0}(p), \rho=\varphi_{0}(p)
$$

while the 3 -wave curve $\mathcal{C}^{3}\left(\mathbf{U}_{0}\right)$ of states $\mathbf{U}$ which can be connected on the left by an admissible 3 -wave is given by

$$
u=u_{0}+\Phi_{0}(p), \rho=\varphi_{0}(p) .
$$

Now, for $i=1,3$, we denote by $\Gamma^{i}\left(\mathbf{U}_{0}\right)$ the projection onto the $(u, p)$-plane of the $i$-wave curve $\mathcal{C}^{i}\left(\mathbf{U}_{0}\right)$. Hence, the $\Gamma$-curves are defined by

$$
\left\{\begin{array}{l}
\Gamma^{1}\left(\mathbf{U}_{0}\right): u=u_{0}-\Phi_{0}(p) \\
\Gamma^{3}\left(\mathbf{U}_{0}\right): u=u_{0}+\Phi_{0}(p) .
\end{array}\right.
$$

Note that $\Phi_{0}$ is a strictly increasing function with

$$
\Phi_{0}(0)=-\int_{0}^{p_{0}} \frac{d p}{(\rho c)\left(p, s_{0}\right)} .
$$

If we consider the case of a polytropic ideal gas whose equation of state is of the form

$$
p=(\gamma-1) \rho \varepsilon,
$$

then we have on the one hand,

$$
h_{0}(p)=\tau_{0} \frac{\mu^{2} p+p_{0}}{p+\mu^{2} p_{0}}, \mu^{2}=\frac{\gamma-1}{\gamma+1}
$$

so that

$$
\sqrt{\left(p-p_{0}\right)\left(\tau_{0}-h_{0}(p)\right)}=\left|p-p_{0}\right| \sqrt{\frac{1-\mu^{2}}{\rho_{0}\left(p+\mu^{2} p_{0}\right)}} .
$$

On the other hand, since the polytropic equation of state may be equivalently written in the form

$$
p=A(s) \rho^{\gamma},
$$


we find

$$
\rho\left(p, s_{0}\right)=\rho_{0}\left(\frac{p}{p_{0}}\right)^{\frac{1}{\gamma}}
$$

and since $c=\sqrt{\frac{\gamma p}{\rho}}$,

which yields

$$
c\left(p, s_{0}\right)=c_{0}\left(\frac{p}{p_{0}}\right)^{\frac{\gamma-1}{2 \gamma}}
$$

$$
\int_{p_{0}}^{p} \frac{d p}{(\rho c)\left(p, s_{0}\right)}=\frac{2}{\gamma-1}\left(c\left(p, s_{0}\right)-c_{0}\right)=\frac{2}{\gamma-1} c_{0}\left(\left(\frac{p}{p_{0}}\right)^{\frac{\gamma-1}{2 \gamma}}-1\right) .
$$

Therefore, we obtain for a polytropic gas,

$$
\Phi_{0}(p)= \begin{cases}\frac{2}{\gamma-1}\left(c\left(p, s_{0}\right)-c_{0}\right), & \text { if } p \leq p_{0}, \\ \left(p-p_{0}\right) \sqrt{\frac{1-\mu^{2}}{\rho_{0}\left(p+\mu^{2} p_{0}\right)},} & \text { if } p>p_{0},\end{cases}
$$

and

We have in that case

$$
\varphi_{0}(p)= \begin{cases}\rho_{0}\left(\frac{p}{p_{0}}\right)^{\frac{1}{\gamma}}, & \text { if } p \leq p_{0} \\ \rho_{0} \frac{p+\mu^{2} p_{0}}{\mu^{2} p+p_{0}}, & \text { if } p>p_{0} .\end{cases}
$$

$$
\Phi_{0}(0)=-\frac{2 c_{0}}{\gamma-1} .
$$

Let us lastly recall that the states $\mathbf{U}$ which can be connected to $\mathbf{U}_{0}$ on the right or on the left by a 2-contact discontinuity are characterized by

$$
u=u_{0}, p=p_{0} .
$$

We are now in a position to study the signs of the wave speeds. We first consider the 1 -waves which connect a state $\mathbf{U}$ to $\mathbf{U}_{0}$ on the right $\left(\mathbf{U} \in \mathcal{C}^{1}\left(\mathbf{U}_{0}\right)\right)$. We begin with the 1 -shock waves. Let us denote by $\sigma$ the speed of such a shock; we observe that $\sigma=\sigma(p)$ may be parametrized by $p$ as $\mathbf{U}$ varies along the shock part $\mathcal{C}_{s}^{1}\left(\mathbf{U}_{0}\right)$ of the wave curve $\mathcal{C}^{1}\left(\mathbf{U}_{0}\right)$. Then we can state

Proposition 1. Assume the hypothesis H.1. The following properties hold:

(i) for $u_{0}<c_{0}$,we have $\sigma=\sigma(p)<0$ along $\mathcal{C}_{s}^{1}\left(\mathbf{U}_{0}\right)$;

(ii) for $u_{0} \geq c_{0}$, there exists a unique $p_{s}^{1}=p_{s}^{1}\left(\mathbf{U}_{0}\right) \geq p_{0}$ such that

$$
\sigma\left(p_{s}^{1}\right)=0
$$

and we have

$$
\begin{cases}\sigma(p)>0, & \text { if } p_{0}<p<p_{s}^{1} \\ \sigma(p)<0, & \text { if } p>p_{s}^{1}\end{cases}
$$

Moreover $p_{s}^{1}$ is the unique solution of the equation

$$
\rho_{0} u_{0} h_{0}(p)=u_{0}-\Phi_{0}(p), p \geq p_{0} .
$$


Proof. Using the Rankine-Hugoniot jump conditions, we obtain on the one hand from the Hugoniot equation

$$
\tau=h_{0}(p)
$$

and on the other hand from the mass conservation 2

$$
[\rho u]=\sigma[\rho] ;
$$

hence

$$
\rho u=\rho_{0} u_{0}+\sigma\left(\rho-\rho_{0}\right) .
$$

Since $\rho>\rho_{0}$ for such an admissible shock, we have

$$
\left\{\begin{array}{l}
\sigma=0 \Longleftrightarrow \rho u=\rho_{0} u_{0}, \\
\operatorname{sgn}(\sigma)=\operatorname{sgn}\left(\rho u-\rho_{0} u_{0}\right), \rho=\frac{1}{h_{0}(p)} .
\end{array}\right.
$$

This leads us to consider the curve of equation

$$
u=\rho_{0} u_{0} h_{0}(p) .
$$

In order to determine the sign of $\sigma$, we need only to find the intersection point(s) of this curve with the shock part $\mathcal{C}_{s}^{1}\left(\mathbf{U}_{0}\right)$ of the 1 -wave curve $\mathcal{C}^{1}\left(\mathbf{U}_{0}\right)$ or equivalently to solve the equation

$$
\rho_{0} u_{0} h_{0}(p)=u_{0}-\sqrt{\left(p-p_{0}\right)\left(\tau_{0}-h_{0}(p)\right)}, p \geq p_{0},
$$

which we write in the form

$$
\frac{u_{0}}{\tau_{0}}\left(\tau_{0}-h_{0}(p)\right)=\sqrt{\left(p-p_{0}\right)\left(\tau_{0}-h_{0}(p)\right)}, p \geq p_{0} .
$$

Since $\tau=h_{0}(p) \leq \tau_{0}$ for $p \geq p_{0}$, we must have $u_{0} \geq 0$ for the above equation to possess a solution. Let us then assume $u_{0} \geq 0$; we have to solve

$$
u_{0}=\tau_{0} \sqrt{\frac{p-p_{0}}{\tau_{0}-h_{0}(p)}} \Longleftrightarrow u_{0}^{2}=\tau_{0}^{2} \frac{p-p_{0}}{\tau_{0}-h_{0}(p)} .
$$

If we define the function

$$
f(p)=\tau_{0}^{2} \frac{p-p_{0}}{\tau_{0}-h_{0}(p)}
$$

we observe that

$$
f^{\prime}(p)=\frac{\tau_{0}^{2}}{\left(\tau_{0}-h_{0}(p)\right)^{2}}\left(\tau_{0}-h_{0}(p)+\left(p-p_{0}\right) h_{0}^{\prime}(p)\right)>0
$$

by the strict convexity of the function $h_{0}$. In addition, we have (cf. Lemma 1 below)

$$
\begin{gathered}
f\left(p_{0}\right)=-\frac{\tau_{0}^{2}}{h_{0}^{\prime}\left(p_{0}\right)}=c_{0}^{2}, \\
\lim _{p \rightarrow+\infty} f(p)=+\infty .
\end{gathered}
$$

Hence, for $p \geq p_{0}$, the equation

$$
f(p)=u_{0}^{2}
$$

\footnotetext{
${ }^{2}$ As usual $[\varphi]=\varphi-\varphi_{0}$ denotes the jump of a quantity $\varphi$ at a discontinuity.
} 
has a unique solution $p=p_{s}^{1}$ for $u_{0} \geq c_{0}$ and no solution for $u_{0}<c_{0}$. Since, for $p \geq p_{0}$, the expression

$$
\rho u-\rho_{0} u_{0}=\left(\frac{1}{h_{0}(p)}-\frac{1}{h_{0}\left(p_{0}\right)}\right)\left(u_{0}-\tau_{0} \frac{\Phi_{0}(p)}{\tau_{0}-h_{0}(p)}\right)
$$

has the sign of

$$
u_{0}-\tau_{0} \frac{\Phi_{0}(p)}{\tau_{0}-h_{0}(p)}=u_{0}-\tau_{0} \sqrt{\frac{p-p_{0}}{\tau_{0}-h_{0}(p)}},
$$

we find that $\rho u-\rho_{0} u_{0}<0$ for $p$ large enough, which proves our assertions.

It remains to check

Lemma 1. We have

$$
h_{0}^{\prime}\left(p_{0}\right)=-\frac{\tau_{0}^{2}}{c_{0}^{2}}
$$

Proof. We indicate the proof for the reader's convenience. By differentiating Hugoniot's equation, we obtain

$$
\partial_{\tau} \varepsilon(\tau, p) h_{0}^{\prime}(p)+\partial_{p} \varepsilon(\tau, p)+\frac{p}{2}\left(h_{0}(p)-\tau_{0}\right)+\frac{1}{2}\left(p+p_{0}\right) h_{0}^{\prime}(p)=0, \tau=h_{0}(p),
$$

which yields

$$
h_{0}^{\prime}\left(p_{0}\right)=-\frac{\partial_{p} \varepsilon\left(\tau_{0}, p_{0}\right)}{\partial_{\tau} \varepsilon\left(\tau_{0}, p_{0}\right)+p_{0}} .
$$

But, as a consequence of the thermodynamic relation

$$
d \varepsilon=T d s-p d \tau=\partial_{\tau} \varepsilon d \tau+\partial_{p} \varepsilon d p,
$$

we find

and therefore

$$
d p=\frac{1}{\partial_{p} \varepsilon}\left(-\left(\partial_{\tau} \varepsilon+p\right) d \tau+T d s\right)
$$

$$
\frac{c^{2}}{\tau^{2}}=-\partial_{\tau} \varepsilon_{s}=\frac{\partial_{\tau} \varepsilon+p}{\partial_{p} \varepsilon}
$$

which proves the lemma.

We next consider the 1-rarefaction waves which connect $\mathbf{U}$ to the state $\mathbf{U}_{0}$ on the right. As $\mathbf{U}$ varies along the rarefaction part $\mathcal{C}_{r}^{1}\left(\mathbf{U}_{0}\right)$ of the wave curve $\mathcal{C}^{1}\left(\mathbf{U}_{0}\right)$, we want to determine the sign of the speed $u-c\left(p, s_{0}\right)$ of the right edge of the fan of such a rarefaction. Again, we assume a simplifying hypothesis:

H.2. The function $p \mapsto c(p, s)$ is monotonically increasing with

$$
\lim _{p \rightarrow 0} c(p, s)=0 .
$$

REMARK 1. Since

$$
\begin{aligned}
\partial_{p} c(p, s) & =\frac{1}{2 c} \partial_{p} c^{2}(p, s)=\frac{1}{2 c} \partial_{p}\left(\partial_{\rho} p(\rho, s)\right) \\
& =\frac{1}{2 c} \partial_{\rho \rho}^{2} p(\rho, s) \partial_{p} \rho(p, s)=\frac{1}{2 c^{3}} \partial_{\rho \rho}^{2} p(\rho, s),
\end{aligned}
$$

we obtain that the function $p \mapsto c(p, s)$ is decreasing as soon as the function $\rho \mapsto p(\rho, s)$ is convex, which is a property shared by standard gases. Indeed, the assumptions on the function $p \mapsto c(p, s)$ are trivially satisfied for a polytropic gas. 
Proposition 2. Assume the hypothesis H.2. Then the following properties hold:

(i) for $u_{0}<\Phi_{0}(0)$, we have $u-c\left(p, s_{0}\right)<0$ along $\mathcal{C}_{r}^{1}\left(\mathbf{U}_{0}\right)$;

(ii) for $\Phi_{0}(0) \leq u_{0} \leq c_{0}$, there exists a unique $p_{r}^{1}=p_{r}^{1}\left(\mathbf{U}_{0}\right) \in\left[0, p_{0}\right]$ such that

$$
c\left(p_{r}^{1}, s_{0}\right)=u_{0}-\Phi_{0}\left(p_{r}^{1}\right)=u_{0}-\int_{p_{0}}^{p_{r}^{1}} \frac{d p}{(\rho c)\left(p, s_{0}\right)}
$$

and we have

$$
\left\{\begin{array}{l}
u-c\left(p, s_{0}\right)>0, \quad \text { if } p<p_{r}^{1}, \\
u-c\left(p, s_{0}\right)<0, \quad \text { if } p_{r}^{1}<p<p_{0}
\end{array}\right.
$$

(iii) for $u_{0}>c_{0}$, we have $u-c\left(p, s_{0}\right)>0$ along $\mathcal{C}_{r}^{1}\left(\mathbf{U}_{0}\right)$.

Proof. In order to determine the sign of $u-c\left(p, s_{0}\right)$, we need to find the intersection point(s) of the curve $u=c\left(p, s_{0}\right)$ with the curve $\mathcal{C}_{r}^{1}\left(\mathbf{U}_{0}\right)$ or equivalently to solve the equation

$$
c\left(p, s_{0}\right)=u_{0}-\Phi_{0}(p)=u_{0}-\int_{p_{0}}^{p} \frac{d p}{(\rho c)\left(p, s_{0}\right)}, 0 \leq p \leq p_{0} .
$$

Clearly, as $p$ varies from 0 to $p_{0}, c\left(p, s_{0}\right)$ increases from 0 to $c_{0}$ while $u_{0}-\Phi_{0}(p)$ decreases strictly from $u_{0}-\Phi_{0}$ to $u_{0}$. Hence the above equation has a solution which is indeed unique if and only if

$$
\Phi_{0}(0) \leq u_{0} \leq c_{0}
$$

Since by (35)

$$
\lim _{p \rightarrow 0} u-c\left(p, s_{0}\right)=u_{0}-\Phi_{0}(0),
$$

$u-c\left(p, s_{0}\right)$ remains $<0$ in case (i), passes from $<0$ to $>0$ as $p$ crosses $p_{r}^{1}$ in case (ii) and remains $>0$ in case (iii).

To summarize Propositions 1 and 2, we obtain the diagrams of Fig. 1, Defining

$$
\begin{aligned}
& u_{s}^{1}=u_{s}^{1}\left(\mathbf{U}_{0}\right)=u_{0}-\Phi_{0}\left(p_{s}^{1}\right)=\rho_{0} u_{0} h_{0}\left(p_{s}^{1}\right), \\
& u_{r}^{1}=u_{r}^{1}\left(\mathbf{U}_{0}\right)=u_{0}-\Phi_{0}\left(p_{r}^{1}\right)=c\left(p_{r}^{1}, s_{0}\right),
\end{aligned}
$$

we set

$$
u_{\text {max }}^{1}=u_{\text {max }}^{1}\left(\mathbf{U}_{0}\right)= \begin{cases}u_{s}^{1}, & \text { if } u_{0} \geq c_{0}, \\ u_{r}^{1}, & \text { if } \Phi_{0}(0) \leq u_{0} \leq c_{0} .\end{cases}
$$

Then it follows from Propositions 1 and 2 that the part $\mathcal{C}_{-}^{1}\left(\mathbf{U}_{0}\right)$ of the wave curve $\mathcal{C}^{1}\left(\mathbf{U}_{0}\right)$ of all states $\mathbf{U}$ which can be connected to $\mathbf{U}_{0}$ on the right by a (nontrivial) 1-wave whose speed is nonpositive 3 is given by

$$
\mathcal{C}_{-}^{1}\left(\mathbf{U}_{0}\right)= \begin{cases}\mathcal{C}^{1}\left(\mathbf{U}_{0}\right) \backslash\left\{\mathbf{U}_{0}\right\}, & \text { if } u_{0} \leq \Phi_{0}(0), \\ \left\{\mathbf{U} \in \mathcal{C}^{1}\left(\mathbf{U}_{0}\right) \backslash\left\{\mathbf{U}_{0}\right\} ; u \leq u_{\max }^{1}\left(\mathbf{U}_{0}\right)\right\}, & \text { if } u_{0} \geq \Phi_{0}(0) .\end{cases}
$$

Since, for $u_{0} \leq \Phi_{0}(0)$, the velocity $u$ of any state $\mathbf{U} \in \mathcal{C}^{1}\left(\mathbf{U}_{0}\right)$ is less than $\Phi_{0}(0)$, it appears convenient to set

$$
u_{\text {max }}^{1}\left(\mathbf{U}_{0}\right)=u_{0}-\Phi_{0}(0)<0 \text { if } u_{0}<\Phi_{0}(0)
$$

\footnotetext{
${ }^{3} \mathrm{~A}$ rarefaction wave is said to have a nonnegative (resp. a nonpositive) speed if its minimum (resp. maximum) speed is nonnegative (resp. nonpositive).
} 


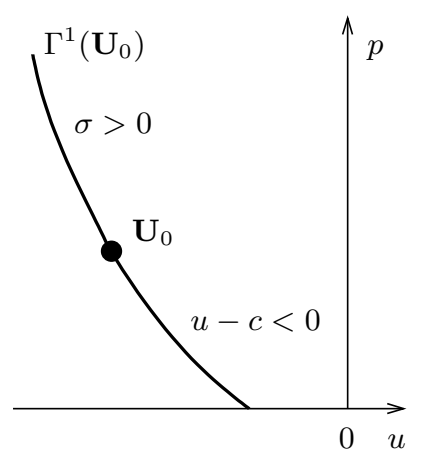

$u_{0}<\Phi_{0}(0)$
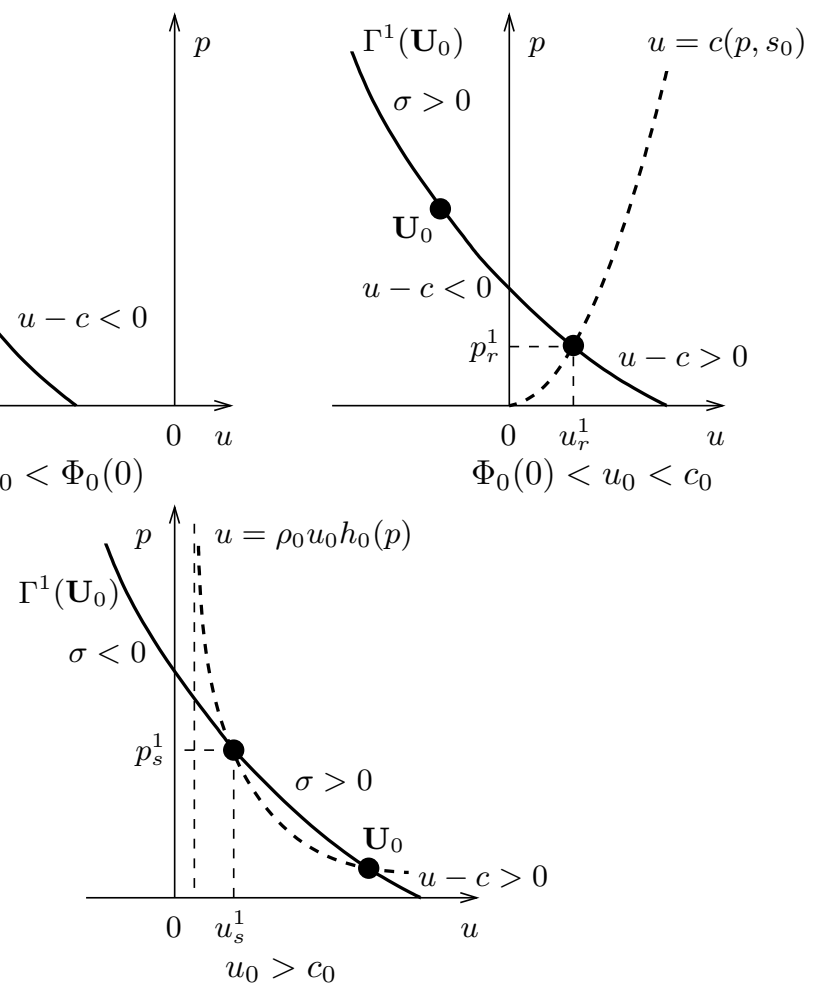

FIG. 1. Signs of the wave speeds of $\mathcal{C}^{1}\left(\mathbf{U}_{0}\right)$.

so that $\mathcal{C}_{-}^{1}\left(\mathbf{U}_{0}\right)$ may be equivalently characterized by

$$
\mathcal{C}_{-}^{1}\left(\mathbf{U}_{0}\right)=\left\{\mathbf{U} \in \mathcal{C}^{1}\left(\mathbf{U}_{0}\right) ; \rho \neq \rho_{0}, u \leq u_{\max }^{1}\left(\mathbf{U}_{0}\right)\right\}
$$

Let us next study the 3 -waves which connect a state $\mathbf{U}$ to the state $\mathbf{U}_{0}$ on the left $\left(\mathbf{U} \in \mathcal{C}^{3}\left(\mathbf{U}_{0}\right)\right)$. We first consider the shock waves, and again we denote by $\sigma=\sigma(p)$ the speed of such a shock when $\mathbf{U}$ varies along the shock part $\mathcal{C}_{s}^{3}\left(\mathbf{U}_{0}\right)$ of $\mathcal{C}^{3}\left(\mathbf{U}_{0}\right)$.

Proposition 3. Assume the hypothesis H.1. The following properties hold:

(i) for $u_{0}>-c_{0}$, we have $\sigma(p)>0$ along $\mathcal{C}_{s}^{3}\left(\mathbf{U}_{0}\right)$;

(ii) for $u_{0} \leq-c_{0}$, there exists a unique $p_{s}^{3} \geq p_{0}$ such that

$$
\sigma\left(p_{s}^{3}\right)=0
$$

and we have

$$
\begin{cases}\sigma(p)<0, & \text { if } p_{0}<p<p_{s}^{3} \\ \sigma(p)>0, & \text { if } p>p_{s}^{3} .\end{cases}
$$

Moreover $p_{s}^{3}$ is the unique solution of the equation

$$
\rho_{0} u_{0} h_{0}(p)=u_{0}+\Phi_{0}(p), p \geq p_{0} .
$$


Proof. The proof mimics that of Proposition 1. Here we have to find the intersection of the curve of equation $u=\rho_{0} u_{0} h_{0}(p)$ with $\mathcal{C}_{s}^{3}\left(\mathbf{U}_{0}\right)$ or equivalently to solve

$$
\frac{u_{0}}{\tau_{0}}\left(\tau_{0}-h_{0}(p)=-\sqrt{\left(p-p_{0}\right) \tau_{0}-h_{0}(p)}\right), p \geq p_{0} .
$$

We pass to the 3-rarefaction waves which connect $\mathbf{U}$ to $\mathbf{U}_{0}$ on the left. Here we want to determine the sign of the speed $u+c\left(p, s_{0}\right)$ of the left edge of the fan of such a rarefaction as $\mathbf{U}$ varies along the rarefaction part $\mathcal{C}_{r}^{3}\left(\mathbf{U}_{0}\right)$ of the wave curve $\mathcal{C}^{1}\left(\mathbf{U}_{0}\right)$. We can state the analogue of Proposition 2.

Proposition 4. Assume the hypothesis H.2. Then the following properties hold:

(i) for $u_{0}>\int_{0}^{p_{0}} \frac{d p}{(\rho c)\left(p, s_{0}\right)}$, we have $u+c\left(p, s_{0}\right)>0$ along $\mathcal{C}_{r}^{3}\left(\mathbf{U}_{0}\right)$;

(ii) for $-c_{0} \leq u_{0} \leq \int_{0}^{p_{0}} \frac{d p}{(\rho c)\left(p, s_{0}\right)}$, there exists a unique $p_{r}^{3} \in\left[0, p_{0}\right]$ such that

$$
-c\left(p_{r}^{3}, s_{0}\right)=u_{0}+\Phi_{0}\left(p_{r}^{3}\right)=u_{0}+\int_{p_{0}}^{p_{r}^{3}} \frac{d p}{(\rho c)\left(p, s_{0}\right)}
$$

and we have

$$
\begin{cases}u+c\left(p, s_{0}\right)<0, & \text { if } p<p_{r}^{3} \\ u+c\left(p, s_{0}\right)>0, & \text { if } p>p_{r}^{3}\end{cases}
$$

(iii) for $u_{0}<-c_{0}$, we have $u+c\left(p, s_{0}\right)<0$ along $\mathcal{C}_{r}^{3}\left(\mathbf{U}_{0}\right)$.

To summarize Propositions 3 and 4 , we obtain the diagrams of Fig. 2.

Defining

$$
\begin{gathered}
u_{s}^{3}=u_{s}^{3}\left(\mathbf{U}_{0}\right)=u_{0}+\Phi_{0}\left(p_{s}^{3}\right)=\rho_{0} u_{0} h_{0}\left(p_{s}^{3}\right), \\
u_{r}^{3}=u_{r}^{3}\left(\mathbf{U}_{0}\right)=u_{0}+\Phi_{0}\left(p_{r}^{3}\right)=-c\left(p_{r}^{3}, s_{0}\right),
\end{gathered}
$$

we set

$$
u_{\text {min }}^{3}=u_{\text {min }}^{3}\left(\mathbf{U}_{0}\right)= \begin{cases}u_{s}^{3}, & \text { if } u_{0} \leq-c_{0}, \\ u_{r}^{3}, & \text { if }-c_{0} \leq u_{0} \leq-\Phi_{0}(0), \\ u_{0}+\Phi_{0}(0), & \text { if } u_{0} \geq-\Phi_{0}(0) .\end{cases}
$$

Then it follows from Propositions 3 and 4 that the part $\mathcal{C}_{+}^{3}\left(\mathbf{U}_{0}\right)$ of the wave curve $\mathcal{C}^{3}\left(\mathbf{U}_{0}\right)$ of all states that can be connected to $\mathbf{U}_{0}$ on the left by a (nontrivial) 3-wave whose speed is nonnegative is given by

$$
\mathcal{C}_{+}^{3}\left(\mathbf{U}_{0}\right)=\left\{\mathbf{U} \in \mathcal{C}^{3}\left(\mathbf{U}_{0}\right) ; \rho \neq \rho_{0}, u \geq u_{\min }^{3}\left(\mathbf{U}_{0}\right)\right\} .
$$

3. Some useful sets of the state space. Consider again the solution $\mathbf{W}(x / t$; $\mathbf{U}_{G}, \mathbf{U}_{D}$ ) of the classical Riemann problem for the gas dynamics system:

$$
\left\{\begin{array}{l}
\partial_{t} \mathbf{U}+\partial_{x} \mathbf{F}(\mathbf{U})=\mathbf{0}, x \in \mathbb{R}, t>0, \\
\mathbf{U}(x, 0)= \begin{cases}\mathbf{U}_{G}, & \text { if } x<0, \\
\mathbf{U}_{D}, & \text { if } x>0 .\end{cases}
\end{array}\right.
$$




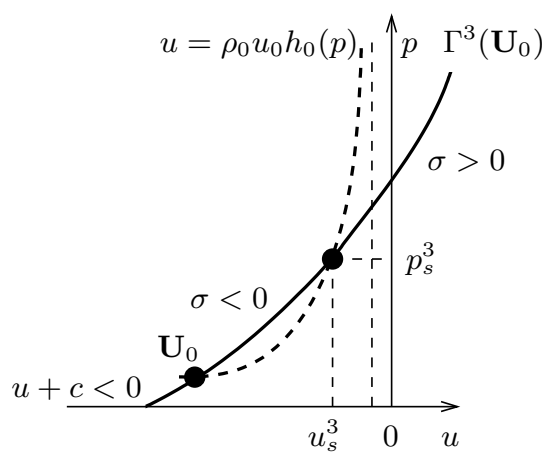

$u_{0}>-c_{0}$

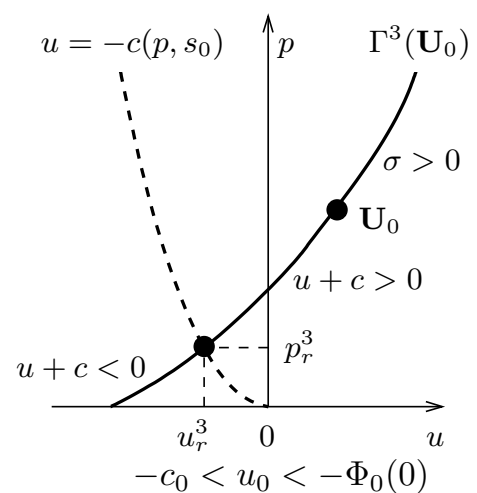

$-c_{0}<u_{0}<-\Phi_{0}(0)$

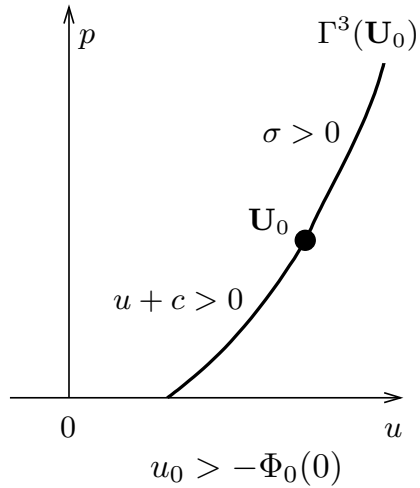

FIG. 2. Signs of the wave speeds of $\mathcal{C}^{3}\left(\mathbf{U}_{0}\right)$.

For any state $\mathbf{U}_{0}$ of the state space $\Omega$, we introduce the following two sets which will play an essential role in all of the remainder of this paper:

$$
\mathcal{E}^{+}\left(\mathbf{U}_{0}\right)=\left\{\mathbf{W}\left(0_{-} ; \mathbf{U}, \mathbf{U}_{0}\right) ; \mathbf{U} \in \Omega\right\}
$$

and

$$
\mathcal{E}^{-}\left(\mathbf{U}_{0}\right)=\left\{\mathbf{W}\left(0_{+} ; \mathbf{U}_{0}, \mathbf{U}\right) ; \mathbf{U} \in \Omega\right\} .
$$

In this section, we want to give a characterization and a geometric description of these sets.

We begin by deriving a necessary and sufficient condition for the solution $\mathbf{W}(x / t$; $\left.\mathbf{U}_{G}, \mathbf{U}_{D}\right)$ to consist of waves with nonnegative velocities. Besides the trivial case where $\mathbf{U}_{G}=\mathbf{U}_{D}$, we need to examine the following three situations:

(i) $\mathbf{U}_{G}$ is connected to $\mathbf{U}_{D}$ by a (nontrivial) 3-wave;

(ii) $\mathbf{U}_{G}$ is connected to $\mathbf{U}_{D}$ by a (nontrivial) 2-contact discontinuity and a 3-wave;

(iii) $\mathbf{U}_{G}$ is connected to $\mathbf{U}_{D}$ by a (nontrivial) 1-wave, a 2-contact discontinuity and a 3-wave.

The situation (i) corresponds indeed to $\mathbf{U}_{G} \in \mathcal{C}_{+}^{3}\left(\mathbf{U}_{D}\right)$, where $\mathcal{C}_{+}^{3}\left(\mathbf{U}_{D}\right)$ is defined as in (43).

Next, given a state $\mathbf{U}_{0}$, we look for all states $\mathbf{U}$ which can be connected to $\mathbf{U}_{0}$ on the left by a (nontrivial) 2 -contact discontinuity with a nonnegative speed and a 3 -wave. 
If we denote by $\mathbf{U}_{0}^{*}$ the intermediate state between $\mathbf{U}$ and $\mathbf{U}_{0}$, we know that $\mathbf{U}$ and $\mathbf{U}_{0}^{*}$ have the same velocity $u_{0}^{*} \geq 0$ and the same pressure $p_{0}^{*}$ while the density of $\mathbf{U}$ is arbitrary. Hence if we introduce the cylindrical surface

$$
\mathcal{S}_{+}^{3}\left(\mathbf{U}_{0}\right)=\left\{\mathbf{U} \in \Omega ; \Upsilon=(u, p) \in \Gamma^{3}\left(\mathbf{U}_{0}\right), \rho \neq \varphi_{0}(p), u \geq 0\right\},
$$

we find that such states exactly generate the surface $\mathcal{S}_{+}^{3}\left(\mathbf{U}_{0}\right)$. Therefore the situation (ii) corresponds to $\mathbf{U}_{G} \in \mathcal{S}_{+}^{3}\left(\mathbf{U}_{D}\right)$.

The situation (iii) is somewhat more complicated to characterize. Suppose that $\mathbf{U}_{G}$ is indeed connected to $\mathbf{U}_{D}$ by a 1-wave followed by a 2-contact discontinuity and a 3wave (these two last waves may not exist). First we notice that, if the 1-wave has a nonnegative speed, we have necessarily

$$
u_{G} \geq c_{G} .
$$

This is obvious if the 1-wave is a rarefaction and it follows from the Lax entropy condition if the 1-wave is a shock. Then, by Proposition 1, we know that there exists a unique state $\mathbf{U}_{s}^{1}=\mathbf{U}_{s}^{1}\left(\mathbf{U}_{G}\right) \in \mathcal{C}^{1}\left(\mathbf{U}_{G}\right)$ which is connected to $\mathbf{U}_{G}$ by a stationary shock. This state $\mathbf{U}_{s}^{1}$ is a solution of

$$
u_{s}^{1}=u_{G}-\Phi_{G}\left(p_{s}^{1}\right)=\rho_{G} u_{G} h_{G}\left(p_{s}^{1}\right)
$$

and satisfies: $u_{s}^{1} \leq u_{G}, p_{s}^{1} \geq p_{G}$ (cf. Fig. 3).

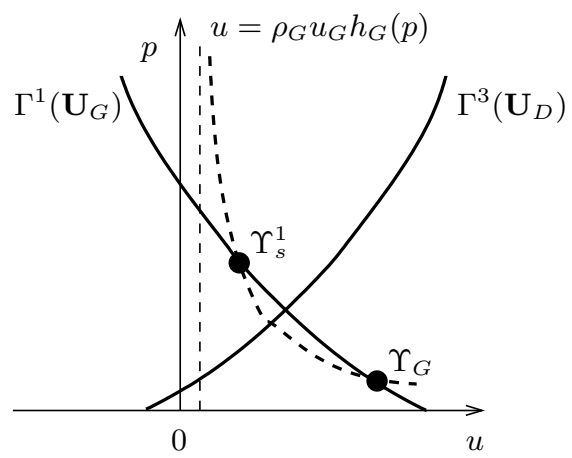

FIG. 3. The wave curves $\mathcal{C}^{1}\left(\mathbf{U}_{G}\right)$ and $\mathcal{C}^{3}\left(\mathbf{U}_{D}\right)$.

We can now state

Proposition 5. Assume that the solution of the Riemann problem (44) possesses a 1-wave. A necessary and sufficient condition for this wave to have a nonnegative speed reads

$$
\left\{\begin{array}{l}
u_{G} \geq c_{G} \\
u_{s}^{1} \leq u_{D}+\Phi_{D}\left(p_{s}^{1}\right)
\end{array}\right.
$$

Proof. Suppose first that the 3 -wave exists. We thus consider the 3 -wave curve $\mathcal{C}^{3}\left(\mathbf{U}_{D}\right)$. If

$$
u_{D}+\Phi_{D}(0) \leq u_{G}-\Phi_{G}(0),
$$


its projection $\Gamma^{3}\left(\mathbf{U}_{D}\right)$ onto the $(u, p)$-plane intersects $\Gamma^{1}\left(\mathbf{U}_{G}\right)$ at a point $\Upsilon_{*}=\left(u_{*}, p_{*}\right)$. Now, using again Proposition 1, the 1-wave has a nonnegative velocity if and only if the point $\Upsilon_{*}$ is located on $\Gamma^{1}\left(\mathbf{U}_{G}\right)$ below the projection $\Upsilon_{s}^{1}=\left(u_{s}^{1}, p_{s}^{1}\right)$ of $\mathbf{U}_{s}^{1}$ onto the $(u, p)$-plane or equivalently $\Upsilon_{s}^{1}$ is located above the curve $\Gamma^{3}\left(\mathbf{U}_{D}\right)$ (cf. again Fig. 3), i.e.,

$$
u_{s}^{1} \leq u_{D}+\Phi_{D}\left(p_{s}^{1}\right) .
$$

On the other hand, if

$$
u_{D}+\Phi_{D}(0)>u_{G}-\Phi_{G}(0)
$$

then $\Gamma^{3}\left(\mathbf{U}_{D}\right)$ does not intersect $\Gamma^{1}\left(\mathbf{U}_{G}\right)$ and a vacuum occurs. However, in this case where the inequality $u_{s}^{1} \leq u_{D}+\Phi_{D}\left(p_{s}^{1}\right)$ clearly holds, the 1-wave is a rarefaction with a nonnegative speed.

Suppose next that the 3-wave does not exist. This means that $\Upsilon_{*}=\Upsilon_{D}$ and again $\Upsilon_{*}$ is located below $\Upsilon_{s}^{1}$ on the curve $\Gamma^{1}\left(\mathbf{U}_{G}\right)$ if and only if $u_{s}^{1} \leq u_{D}+\Phi_{D}\left(p_{s}^{1}\right)$. This proves the result.

REMARK 2. Note that a necessary and sufficient condition for the 1-wave to have a positive speed is given by

$$
\left\{\begin{array}{l}
u_{G} \geq c_{G}, \\
u_{s}^{1}<u_{D}+\Phi_{D}\left(p_{s}^{1}\right) .
\end{array}\right.
$$

Indeed, the proof is identical to that of the above result where the only difference that we have to exclude is the case where the 1-wave is a stationary shock connecting $\mathbf{U}_{G}$ and $\mathbf{U}_{1}$, i.e., the case where $u_{s}^{1}=u_{D}+\Phi_{D}\left(p_{s}^{1}\right)$.

Since both $u_{s}^{1}=u_{s}^{1}\left(\mathbf{U}_{G}\right)$ and $p_{s}^{1}=p_{s}^{1}\left(\mathbf{U}_{G}\right)$ are functions of $\mathbf{U}_{G}$, it makes sense to introduce the following subset of $\Omega$ :

$$
\begin{aligned}
\mathcal{V}_{+}^{3}\left(\mathbf{U}_{D}\right)=\left\{\mathbf{U}_{G} ; u_{G} \geq c_{G}, u_{G} \neq u_{D}+\Phi_{D}\left(p_{G}\right),\right. \\
\left.u_{s}^{1}\left(\mathbf{U}_{G}\right) \leq u_{D}+\Phi_{D}\left(p_{s}^{1}\left(\mathbf{U}_{G}\right)\right)\right\} .
\end{aligned}
$$

Hence the condition

$$
\mathbf{U}_{G} \in \mathcal{V}_{+}^{3}\left(\mathbf{U}_{D}\right)
$$

is a necessary and sufficient condition for the (nontrivial) 1-wave of the solution of the Riemann problem (44) to have a nonnegative speed.

We summarize the above results in

Proposition 6. Assume the hypotheses H.1 and H.2. Then a necessary and sufficient condition for the solution of the Riemann problem (44) to consist of waves with nonnegative speeds reads:

$$
\mathbf{U}_{G} \in\left\{\mathbf{U}_{D}\right\} \cup \mathcal{C}_{+}^{3}\left(\mathbf{U}_{D}\right) \cup \mathcal{S}_{+}^{3}\left(\mathbf{U}_{D}\right) \cup \mathcal{V}_{+}^{3}\left(\mathbf{U}_{D}\right) .
$$

We already know that $\mathcal{C}_{+}^{3}\left(\mathbf{U}_{D}\right)$ is a curve and $\mathcal{S}_{+}^{3}\left(\mathbf{U}_{D}\right)$ is a cylindrical surface. It remains to give a geometric description of the set $\mathcal{V}_{+}^{3}\left(\mathbf{U}_{D}\right)$. This can be done fairly easily in the case of a polytropic ideal gas.

Proposition 7. Assume the equation of state (22). Then, for any state $\mathbf{U}_{0} \in \Omega$, the set $\mathcal{V}_{+}^{3}\left(\mathbf{U}_{0}\right)$ is given by

$$
\mathcal{V}_{+}^{3}\left(\mathbf{U}_{0}\right)=\left\{\mathbf{U} \in \Omega ; \rho \geq \mathcal{R}_{0}^{+}(u, p), u \neq u_{0}+\Phi_{0}(p), u>0\right\},
$$

where $\mathcal{R}_{0}^{+}$is the continuous function defined in the following way: 
(i) for $u<u_{0}+\Phi_{0}(p)$, we have

$$
\mathcal{R}_{0}^{+}(u, p)=\frac{\gamma p}{u^{2}}
$$

(ii) for $u>u_{0}+\Phi_{0}(p), \rho=\mathcal{R}_{0}^{+}(u, p)$ is the unique solution of

$$
\Phi_{0}\left(\left(1-\mu^{2}\right) \rho u^{2}-\mu^{2} p\right)-\left(\mu^{2}+\left(1+\mu^{2}\right) \frac{p}{\rho u^{2}}\right) u+u_{0}=0 .
$$

Proof. For the reader's convenience, we keep the notation $\mathbf{U}_{G}, \mathbf{U}_{D}$ and $\mathbf{U}_{s}^{1}$ introduced previously. Recall that $\mathbf{U}_{s}^{1}$ belongs to the shock part $\mathcal{C}_{s}^{1}\left(\mathbf{U}_{G}\right)$ of the wave curve $\mathcal{C}^{1}\left(\mathbf{U}_{G}\right)$. Hence, the relations (48) become, in the case of a polytropic equation of state (22),

$$
u_{s}^{1}=u_{G}-\left(p_{s}^{1}-p_{G}\right) \sqrt{\frac{1-\mu^{2}}{\rho_{G}\left(p_{s}^{1}+\mu^{2} p_{G}\right)}}=\frac{\mu^{2} p_{s}^{1}+p_{G}}{p_{s}^{1}+\mu^{2} p_{G}} u_{G}
$$

so that

$$
\left\{\begin{array}{l}
u_{s}^{1}=\left(\mu^{2}+\left(1+\mu^{2}\right) \frac{p_{G}}{\rho_{G} u_{G}^{2}}\right) u_{G}, \\
p_{s}^{1}=\left(1-\mu^{2}\right) \rho_{G} u_{G}^{2}-\mu^{2} p_{G} .
\end{array}\right.
$$

The first condition in (49), $u_{G} \geq c_{G}$, gives

$$
u_{G}>0, \rho_{G} \geq \frac{\gamma p_{G}}{u_{G}^{2}} .
$$

Thus, given $u_{G}>0, p_{G}>0$, we look for the supplementary constraint imposed on $\rho_{G}$ by the second condition in (49), $u_{s}^{1} \leq u_{D}+\Phi_{D}\left(p_{s}^{1}\right)$. We set

$$
g\left(\rho_{G}\right)=u_{D}+\Phi_{D}\left(p_{s}^{1}\right)-u_{s}^{1} .
$$

Observe that $g$ is a strictly increasing function. Indeed, since $\Phi_{D}^{\prime}>0$ and

$$
\frac{d u_{s}^{1}}{d \rho_{G}}=-\frac{\left(1+\mu^{2}\right) p_{G}}{\rho_{G}^{2} u_{G}}<0, \frac{d p_{s}^{1}}{d \rho_{G}}=\left(1-\mu^{2}\right) u_{G}^{2}>0,
$$

we have

$$
g^{\prime}\left(\rho_{G}\right)=\Phi_{D}^{\prime}\left(p_{s}^{1}\right) \frac{d p_{s}^{1}}{d \rho_{G}}-\frac{d u_{s}^{1}}{d \rho_{G}}>0 .
$$

Let us then compute $g\left(\gamma p_{G} / u_{G}^{2}\right)$. If $\rho_{G}=\gamma p_{G} / u_{G}^{2}$, we have $u_{s}^{1}=u_{G}$ and $p_{s}^{1}=p_{G}$ so that

$$
g\left(\frac{\gamma p_{G}}{u_{G}^{2}}\right)=u_{D}+\Phi_{D}\left(p_{G}\right)-u_{G} .
$$

Assume first that $\left(u_{G}, p_{G}\right)$ satisfies $u_{G} \leq u_{D}+\Phi_{D}\left(p_{G}\right)$. Then $g\left(\gamma p_{G} / u_{G}^{2}\right) \geq 0$ and the second inequality in (49) holds as soon as

$$
\rho_{G} \geq \mathcal{R}_{D}^{+}\left(u_{G}, p_{G}\right)=\frac{\gamma p_{G}}{u_{G}^{2}} .
$$

Assume next $u_{G}>u_{D}+\Phi_{D}\left(p_{G}\right)$. Since $g\left(\rho_{G}\right)$ tends obviously to $+\infty$ as $\rho_{G}$ tends to $+\infty$, there exists a unique

$$
\rho_{G}^{\min }=\mathcal{R}_{D}^{+}\left(u_{G}, p_{G}\right)>\frac{\gamma p_{G}}{u_{G}^{2}}
$$


solution of the nonlinear equation

$$
g\left(\rho_{G}\right)=u_{D}+\Phi_{D}\left(\left(1-\mu^{2}\right) \rho_{G} u_{G}^{2}-\mu^{2} p_{G}\right)-\left(\mu^{2}+\left(1+\mu^{2}\right) \frac{p_{G}}{\rho_{G} u_{G}^{2}}\right) u_{G}=0
$$

and the conditions (49) hold provided that

$$
\rho_{G} \geq \mathcal{R}_{D}^{+}\left(u_{G}, p_{G}\right) .
$$

Note that $\rho_{G}^{\text {min }}=\gamma p_{G} / u_{G}^{2}$ if $u_{G}=u_{D}+\Phi_{D}\left(p_{G}\right)$ so that the function $\left(u_{G}, p_{G}\right) \mapsto$ $\mathcal{R}_{D}^{+}\left(u_{G}, p_{G}\right)$ is indeed continuous along the curve $\Gamma^{3}\left(\mathbf{U}_{D}\right)$.

REMARK 3. The proof of the previous result remains valid as long as one can check that the function $g$ defined by (57) is strictly increasing. Hence a similar geometric description of the volume $\mathcal{V}_{+}^{3}\left(\mathbf{U}_{0}\right)$ holds for any equation of state which ensures this property of the function $g$. It is not obvious at first glance how to characterize such an equation of state. However, the analogue of Theorem 2 can be obtained for a larger class than that of polytropic ideal gases. For instance, this is the case for a stiffened equation of state of Grüneisen type, i.e., of the form

$$
p=(\gamma-1) \rho \varepsilon+c_{r e f}^{2}\left(\rho-\rho_{r e f}\right) .
$$

The details are left to the reader.

Similarly, we can obtain a necessary and sufficient condition for the solution of the Riemann problem (44) to consist of waves with nonpositive speeds. We first introduce the sets $\mathcal{C}_{-}^{1}\left(\mathbf{U}_{0}\right)$ as in (39) and set

$$
\mathcal{S}_{-}^{1}\left(\mathbf{U}_{0}\right)=\left\{\mathbf{U} \in \Omega ; \Upsilon=(u, p) \in \Gamma^{1}\left(\mathbf{U}_{0}\right), u \leq 0, \rho \neq \varphi_{0}(p)\right\} .
$$

Then $\mathcal{C}_{-}^{1}\left(\mathbf{U}_{G}\right)$ (resp. $\mathcal{S}_{-}^{1}\left(\mathbf{U}_{G}\right)$ ) is the set of all states which can be connected to $\mathbf{U}_{G}$ on the right by a (nontrivial) 1-wave with a nonpositive speed (resp. by a 1-wave and a (nontrivial) 2-contact discontinuity with a nonpositive speed).

Consider next the states $\mathbf{U}_{D}$ which can be connected to $\mathbf{U}_{G}$ on the right by a 1-wave, a 2 -contact discontinuity and a 3 -wave with a nonpositive speed. Then we have necessarily $u_{D} \leq-c_{D}$ and, by Proposition 3 , there exists a unique state $\mathbf{U}_{s}^{3}=\mathbf{U}_{s}^{3}\left(\mathbf{U}_{D}\right) \in \mathcal{C}^{3}\left(\mathbf{U}_{D}\right)$ which is connected to $\mathbf{U}_{D}$ by a stationary shock. This state $\mathbf{U}_{s}^{3}$ is a solution of

$$
u_{s}^{3}=u_{D}+\Phi_{D}\left(p_{s}^{3}\right)=\rho_{D} u_{D} h_{D}\left(p_{s}^{3}\right) .
$$

Now we can prove the analogue of Proposition 5 a necessary and sufficient condition for the 3-wave of the solution of the Riemann problem (44) to have a nonpositive speed reads

$$
\left\{\begin{array}{l}
u_{D} \leq-c_{D} \\
u_{s}^{3} \geq u_{G}-\Phi_{G}\left(p_{s}^{3}\right) .
\end{array}\right.
$$

Thus we are led to introduce the volume of $\Omega$ (which excludes trivial 3-waves)

$$
\begin{array}{r}
\mathcal{V}_{-}^{1}\left(\mathbf{U}_{G}\right)=\left\{\mathbf{U}_{D} ; u_{D} \leq-c_{D}, u_{D} \neq u_{G}-\Phi_{G}\left(p_{D}\right)\right. \\
\left.u_{s}^{3}\left(\mathbf{U}_{D}\right) \geq u_{G}-\Phi_{G}\left(p_{s}^{3}\left(\mathbf{U}_{D}\right)\right)\right\}
\end{array}
$$

and we can state 
Proposition 8. Assume the hypotheses H.1 and H.2. Then a necessary and sufficient condition for the solution of the Riemann problem (44) to consist of waves with nonpositive speeds is given by

$$
\mathbf{U}_{D} \in \mathcal{C}_{-}^{1}\left(\mathbf{U}_{G}\right) \cup \mathcal{S}_{-}^{1}\left(\mathbf{U}_{G}\right) \cup \mathcal{V}_{-}^{1}\left(\mathbf{U}_{G}\right) .
$$

Again the volume $\mathcal{V}_{-}^{1}\left(\mathbf{U}_{G}\right)$ has a simple geometric description in the case of a polytropic ideal gas.

Proposition 9. Assume the equation of state (22). Then, for any state $\mathbf{U}_{0} \in \Omega$, the set $\mathcal{V}_{-}^{1}\left(\mathbf{U}_{0}\right)$ is given by

$$
\mathcal{V}_{-}^{1}\left(\mathbf{U}_{0}\right)=\left\{\mathbf{U} \in \Omega ; \rho \geq \mathcal{R}_{0}^{-}(u, p), u \neq u_{0}-\Phi_{0}(p), u<0\right\},
$$

where $\mathcal{R}_{0}^{-}$is the continuous function defined in the following way:

(i) for $u>u_{0}-\Phi_{0}(p)$, we have

$$
\mathcal{R}_{0}^{-}(u, p)=\frac{\gamma p}{u^{2}} ;
$$

(ii) for $u<u_{0}+\Phi_{0}(p), \rho=\mathcal{R}_{0}^{-}(u, p)$ is the unique solution of

$$
\Phi_{0}\left(\left(1-\mu^{2}\right) \rho u^{2}-\mu^{2} p\right)+\left(\mu^{2}+\left(1+\mu^{2}\right) \frac{p}{\rho u^{2}}\right) u-u_{0}=0 .
$$

As a simple application of Propositions 6 and 8 , we obtain

TheOREM 1 . We have for any state $\mathbf{U}_{0}$ of $\Omega$,

$$
\mathcal{E}^{+}\left(\mathbf{U}_{0}\right)=\left\{\mathbf{U}_{0}\right\} \cup \mathcal{C}_{+}^{3}\left(\mathbf{U}_{0}\right) \cup \mathcal{S}_{+}^{3}\left(\mathbf{U}_{0}\right) \cup \mathcal{V}_{+}^{3}\left(\mathbf{U}_{0}\right)
$$

and

$$
\mathcal{E}^{-}\left(\mathbf{U}_{0}\right)=\left\{\mathbf{U}_{0}\right\} \cup \mathcal{C}_{-}^{1}\left(\mathbf{U}_{0}\right) \cup \mathcal{S}_{-}^{1}\left(\mathbf{U}_{0}\right) \cup \mathcal{V}_{-}^{1}\left(\mathbf{U}_{0}\right) .
$$

Proof. We check for instance (65). Recall that

$$
\mathcal{E}^{+}\left(\mathbf{U}_{0}\right)=\left\{\mathbf{W}\left(0_{-} ; \mathbf{U}, \mathbf{U}_{0}\right) ; \mathbf{U} \in \Omega\right\} .
$$

Now the state $\mathbf{X}=\mathbf{W}\left(0_{-} ; \mathbf{U}, \mathbf{U}_{0}\right)$ belongs to $\mathcal{E}^{+}\left(\mathbf{U}_{0}\right)$ if and only if the solution $\mathbf{W}(x / t$; $\left.\mathbf{X}, \mathbf{U}_{0}\right)$ of the Riemann problem for the pair $\left(\mathbf{X}, \mathbf{U}_{0}\right)$ is the constant state $\mathbf{U}_{0}$ or consists of waves with nonnegative speeds. Hence (65) follows from Proposition 6 .

Let us go back to the sets $\mathcal{O}_{L}\left(\mathbf{V}_{D}\right)$ and $\mathcal{O}_{R}\left(\mathbf{V}_{G}\right)$ defined by (8) and (9) respectively. Using (10) and (11) together with (45) and (46), we obtain

$$
\left\{\begin{array}{l}
\mathcal{O}_{L}\left(\mathbf{V}_{D}\right)=\Theta_{L}^{-1}\left(\mathcal{E}_{L}^{+}\left(\Theta_{L}\left(\mathbf{V}_{D}\right)\right)\right) \\
\mathcal{O}_{R}\left(\mathbf{V}_{G}\right)=\Theta_{R}^{-1}\left(\mathcal{E}_{R}^{-}\left(\Theta_{R}\left(\mathbf{V}_{G}\right)\right)\right) .
\end{array}\right.
$$

Using Theorem 1 the $\mathbf{V}$-state coupling conditions (12), (13) can now be detailed in an explicit way: this will be done in Section 5 .

Let us next introduce the sets

$$
\hat{\mathcal{E}}^{+}\left(\mathbf{U}_{0}\right)=\left\{\mathbf{W}\left(0_{+} ; \mathbf{U}, \mathbf{U}_{0}\right) ; \mathbf{U} \in \Omega\right\}
$$

and

$$
\hat{\mathcal{E}}^{-}\left(\mathbf{U}_{0}\right)=\left\{\mathbf{W}\left(0_{-} ; \mathbf{U}_{0}, \mathbf{U}\right) ; \mathbf{U} \in \Omega\right\}
$$


which are slight modifications of the sets $\mathcal{E}^{ \pm}\left(\mathbf{U}_{0}\right)$ and will be of constant use in the next section. We first observe that

$$
\hat{\mathcal{E}}^{ \pm}\left(\mathbf{U}_{0}\right) \subset \mathcal{E}^{ \pm}\left(\mathbf{U}_{0}\right)
$$

Indeed, if $\mathbf{X}=\mathbf{W}\left(0_{+} ; \mathbf{U}, \mathbf{U}_{0}\right) \in \hat{\mathcal{E}}^{+}\left(\mathbf{U}_{0}\right)$, we have

$$
\mathbf{X}=\mathbf{W}\left(0_{-} ; \mathbf{X}, \mathbf{U}_{0}\right) \in \mathcal{E}^{+}\left(\mathbf{U}_{0}\right)
$$

On the other hand, if $\mathbf{X}=\mathbf{W}\left(0_{-} ; \mathbf{U}_{0}, \mathbf{U}\right) \in \hat{\mathcal{E}}^{-}\left(\mathbf{U}_{0}\right)$, we obtain

$$
\mathbf{X}=\mathbf{W}\left(0_{+} ; \mathbf{U}_{0}, \mathbf{X}\right) \in \mathcal{E}^{-}\left(\mathbf{U}_{0}\right) \text {. }
$$

Then we can state

Lemma 2. The set $\mathcal{E}^{+}\left(\mathbf{U}_{0}\right) \backslash \hat{\mathcal{E}}^{+}\left(\mathbf{U}_{0}\right)$ (resp. $\mathcal{E}^{-}\left(\mathbf{U}_{0}\right) \backslash \hat{\mathcal{E}}^{-}\left(\mathbf{U}_{0}\right)$ ) consists of the states $\mathbf{W}\left(0_{-} ; \mathbf{U}, \mathbf{U}_{0}\right)$ (resp. $\mathbf{W}\left(0_{+} ; \mathbf{U}_{0}, \mathbf{U}\right)$ ) associated with a stationary discontinuity.

Proof. Let us characterize the states of $\mathcal{E}^{+}\left(\mathbf{U}_{0}\right) \backslash \hat{\mathcal{E}}^{+}\left(\mathbf{U}_{0}\right)$, the characterization of $\mathcal{E}^{-}\left(\mathbf{U}_{0}\right) \backslash \hat{\mathcal{E}}^{-}\left(\mathbf{U}_{0}\right)$ being similar. We first observe that

$$
\mathbf{W}\left(0_{+} ; \mathbf{U}, \mathbf{U}_{0}\right)=\mathbf{W}\left(0_{-} ; \mathbf{U}, \mathbf{U}_{0}\right)
$$

unless $\mathbf{W}\left(. ; \mathbf{U}, \mathbf{U}_{0}\right)$ presents a stationary discontinuity. Thus assume that the solution $\mathbf{W}\left(. ; \mathbf{U}, \mathbf{U}_{0}\right)$ has a stationary 1-shock. In order to check that $\mathbf{U}=\mathbf{W}\left(0_{-} ; \mathbf{U}, \mathbf{U}_{0}\right) \in$ $\mathcal{E}^{+}\left(\mathbf{U}_{0}\right)$ does not belong to $\hat{\mathcal{E}}^{+}\left(\mathbf{U}_{0}\right)$, we prove that there does not exist any state $\mathbf{U}_{G}$ such that

$$
\mathbf{W}\left(0_{+} ; \mathbf{U}_{G}, \mathbf{U}_{0}\right)=\mathbf{U} \text {. }
$$

If such a state $\mathbf{U}_{G}$ could exist, only two situations would possibly occur:

(i) $\mathbf{U}$ is connected to $\mathbf{U}_{0}$ on the left by waves with positive speeds, which is excluded since the 1-wave is a stationary shock;

(ii) $\mathbf{U}$ is a sonic state of a rarefaction wave, which is again impossible since it contradicts the Lax entropy conditions for the stationary shock.

This proves our assertion.

In the same way, one can check that, if $\mathbf{W}\left(. ; \mathbf{U}, \mathbf{U}_{0}\right)$ has a stationary 2-contact discontinuity or a stationary 3 -shock, then $\mathbf{W}\left(0_{-} ; \mathbf{U}, \mathbf{U}_{0}\right)$ does not belong to $\hat{\mathcal{E}}^{+}\left(\mathbf{U}_{0}\right)$.

It follows from Lemma 2 that, for obtaining $\hat{\mathcal{E}}^{ \pm}\left(\mathbf{U}_{0}\right)$ from $\mathcal{E}^{ \pm}\left(\mathbf{U}_{0}\right)$, we have only to exclude the states corresponding to stationary discontinuity waves. Therefore we introduce the sets $\hat{\mathcal{C}}_{+}^{3}\left(\mathbf{U}_{0}\right), \hat{\mathcal{S}}_{+}^{3}\left(\mathbf{U}_{0}\right), \hat{\mathcal{V}}_{+}^{3}\left(\mathbf{U}_{0}\right)$ and $\hat{\mathcal{C}}_{-}^{1}\left(\mathbf{U}_{0}\right), \hat{\mathcal{S}}_{-}^{1}\left(\mathbf{U}_{0}\right), \hat{\mathcal{V}}_{-}^{1}\left(\mathbf{U}_{0}\right)$ obtained from $\mathcal{C}_{+}^{3}\left(\mathbf{U}_{0}\right), \mathcal{S}_{+}^{3}\left(\mathbf{U}_{0}\right), \mathcal{V}_{+}^{3}\left(\mathbf{U}_{0}\right)$ and $\mathcal{C}_{-}^{1}\left(\mathbf{U}_{0}\right), \mathcal{S}_{-}^{1}\left(\mathbf{U}_{0}\right), \mathcal{V}_{-}^{1}\left(\mathbf{U}_{0}\right)$ respectively by suppressing these states. First, the wave curve $\mathcal{C}^{3}\left(\mathbf{U}_{0}\right)$ contains a state which can be connected to $\mathbf{U}_{0}$ by a stationary shock only if $u_{0}<-c_{0}$. Hence, we have by (42), (43),

$$
\hat{\mathcal{C}}_{+}^{3}\left(\mathbf{U}_{0}\right)=\left\{\mathbf{U} \in \mathcal{C}_{+}^{3}\left(\mathbf{U}_{0}\right) ; u>u_{s}^{3}\left(\mathbf{U}_{0}\right) \text { if } u_{0}<-c_{0}\right\} .
$$

Next, we have to exclude from $\mathcal{S}_{+}^{3}\left(\mathbf{U}_{0}\right)$ the stationary contact discontinuities so that

$$
\hat{\mathcal{S}}_{+}^{3}\left(\mathbf{U}_{0}\right)=\left\{\mathbf{U} \in \mathcal{S}_{+}^{3}\left(\mathbf{U}_{0}\right) ; u>0\right\} .
$$

Then we consider the set $\mathcal{V}_{+}^{3}\left(\mathbf{U}_{D}\right)$. Using Remark 2, we know that the 1-wave of $\mathbf{W}\left(. ; \mathbf{U}_{G}, \mathbf{U}_{D}\right)$ has a positive speed if and only if

$$
\left\{\begin{array}{l}
u_{G} \geq c_{G} \\
u_{s}^{1}\left(\mathbf{U}_{G}\right)<u_{D}+\Phi_{D}\left(p_{s}^{1}\left(\mathbf{U}_{G}\right)\right)
\end{array}\right.
$$


In other words, we have

$$
\hat{\mathcal{V}}_{+}^{3}\left(\mathbf{U}_{D}\right)=\left\{\mathbf{U}_{G} \in \mathcal{V}_{+}^{3}\left(\mathbf{U}_{D}\right) ; u_{s}^{1}\left(\mathbf{U}_{G}\right)<u_{D}+\Phi_{D}\left(p_{s}^{1}\left(\mathbf{U}_{G}\right)\right)\right\} .
$$

Similarly, we introduce the sets

$$
\begin{gathered}
\hat{\mathcal{C}}_{-}^{1}\left(\mathbf{U}_{0}\right)=\left\{\mathbf{U} \in \mathcal{C}_{-}^{1}\left(\mathbf{U}_{0}\right) ; u<u_{s}^{1}\left(\mathbf{U}_{0}\right) \text { if } u_{0}>c_{0}\right\}, \\
\hat{\mathcal{S}}_{-}^{1}\left(\mathbf{U}_{0}\right)=\left\{\mathbf{U} \in \mathcal{S}_{-}^{1}\left(\mathbf{U}_{0}\right) ; u<0\right\}
\end{gathered}
$$

and

$$
\hat{\mathcal{V}}_{-}^{1}\left(\mathbf{U}_{G}\right)=\left\{\mathbf{U}_{D} \in \mathcal{V}_{-}^{1}\left(\mathbf{U}_{G}\right) ; u_{s}^{3}\left(\mathbf{U}_{D}\right)>u_{G}-\Phi_{G}\left(p_{s}^{3}\left(\mathbf{U}_{D}\right)\right)\right\} .
$$

Now, we deduce from Theorem 1 .

TheOREM 2. We have

$$
\hat{\mathcal{E}}^{+}\left(\mathbf{U}_{0}\right)=\left\{\mathbf{U}_{0}\right\} \cup \hat{\mathcal{C}}_{+}^{3}\left(\mathbf{U}_{0}\right) \cup \hat{\mathcal{S}}_{+}^{3}\left(\mathbf{U}_{0}\right) \cup \hat{\mathcal{V}}_{+}^{3}\left(\mathbf{U}_{0}\right)
$$

and

$$
\hat{\mathcal{E}}^{-}\left(\mathbf{U}_{0}\right)=\left\{\mathbf{U}_{0}\right\} \cup \hat{\mathcal{C}}_{-}^{1}\left(\mathbf{U}_{0}\right) \cup \hat{\mathcal{S}}_{-}^{1}\left(\mathbf{U}_{0}\right) \cup \hat{\mathcal{V}}_{-}^{1}\left(\mathbf{U}_{0}\right) .
$$

In the case of a polytropic ideal gas, we have the following geometric descriptions of the sets $\hat{\mathcal{V}}_{+}^{3}\left(\mathbf{U}_{0}\right)$ and $\hat{\mathcal{V}}_{-}^{1}\left(\mathbf{U}_{0}\right)$.

Proposition 10. Assume the equation of state (22). Then, $\hat{\mathcal{V}}_{+}^{3}\left(\mathbf{U}_{0}\right)$ is the set of all states $\mathbf{U} \in \Omega$ which satisfy

$$
u>0, \begin{cases}\rho \geq \frac{\gamma p}{u^{2}}, & \text { if } u<u_{0}+\Phi_{0}(p), \\ \rho>\mathcal{R}_{0}^{+}(u, p), & \text { if } u>u_{0}+\Phi_{0}(p),\end{cases}
$$

while $\hat{\mathcal{V}}_{-}^{1}\left(\mathbf{U}_{0}\right)$ is the set of all states $\mathbf{U} \in \Omega$ which satisfy

$$
u<0, \begin{cases}\rho \geq \frac{\gamma p}{u^{2}}, & \text { if } u>u_{0}-\Phi_{0}(p), \\ \rho>\mathcal{R}_{0}^{-}(u, p), & \text { if } u<u_{0}-\Phi_{0}(p) .\end{cases}
$$

\section{Solutions of the coupled Riemann problem which are V-continuous at} the interface. Let us go back to the $\mathbf{V}$-coupled Riemann problem: we want to solve Eqs. (1), (2) with the $\mathbf{V}$-coupling conditions (12), (13) and the initial condition (14). We look for self-similar solutions $\mathbf{U}=\mathbf{U}(x, t)$ which consist of constant states separated by $L$-waves 4 with negative speeds, by $R$-waves with positive speeds and eventually by a stationary discontinuity at $x=0$. In this section, we restrict ourselves to search for solutions such that $\mathbf{V}$ is continuous at the interface $x=0$, i.e., which satisfy the constraint (15).

It will be convenient to set

$$
\mathbf{V}_{G}=\Theta_{L}^{-1}\left(\mathbf{U}_{G}\right), \quad \mathbf{V}_{D}=\Theta_{R}^{-1}\left(\mathbf{U}_{D}\right)
$$

and, for simplicity, we will use the notation

$$
\hat{\mathcal{E}}_{L}^{-}\left(\mathbf{V}_{G}\right)=\hat{\mathcal{E}}_{L}^{-}\left(\Theta_{L}\left(\mathbf{V}_{G}\right)\right), \hat{\mathcal{E}}_{R}^{+}\left(\mathbf{V}_{D}\right)=\hat{\mathcal{E}}_{R}^{+}\left(\Theta_{R}\left(\mathbf{V}_{D}\right)\right)
$$

\footnotetext{
${ }^{4}$ By $\alpha$-waves, $\alpha=L, R$, we mean waves corresponding to the equation of state $p=p_{\alpha}(\rho, \varepsilon)$.
} 
and similar notation for the various sets of the state space introduced above. Then we look for a self-similar function $\mathbf{V}=\mathbf{V}(x / t)$ which is continuous at $x=0$ and such that $\mathbf{V}(0)$ is connected to $\mathbf{V}_{G}$ on the right by $L$-waves while $\mathbf{V}(0)$ is connected to $\mathbf{V}_{D}$ on the left by $R$-waves. In other words, we have

$$
\mathbf{V}(0) \in \hat{\mathcal{E}}_{L}^{-}\left(\mathbf{V}_{G}\right) \cap \hat{\mathcal{E}}_{R}^{+}\left(\mathbf{V}_{D}\right) .
$$

Conversely, given $\mathbf{V}_{0}$ in this subset (81) of $\Omega_{V}$, there exists by construction at least one solution $\mathbf{V}=\mathbf{V}(x / t)$ to the $\mathbf{V}$-coupled Riemann problem such that $\mathbf{V}$ is continuous at $x=0$ and satisfies $\mathbf{V}(0)=\mathbf{V}_{0}$.

A first step in the construction of such a solution consists in characterizing the set (81). Using (76) and (77), we know that

$$
\hat{\mathcal{E}}_{R}^{+}\left(\mathbf{V}_{D}\right)=\left\{\mathbf{V}_{D}\right\} \cup \hat{\mathcal{C}}_{R,+}^{3}\left(\mathbf{V}_{D}\right) \cup \hat{\mathcal{S}}_{R,+}^{3}\left(\mathbf{V}_{D}\right) \cup \hat{\mathcal{V}}_{R,+}^{3}\left(\mathbf{V}_{D}\right)
$$

and

$$
\hat{\mathcal{E}}_{L}^{-}\left(\mathbf{V}_{G}\right)=\left\{\mathbf{V}_{G}\right\} \cup \hat{\mathcal{C}}_{L,-}^{1}\left(\mathbf{V}_{G}\right) \cup \hat{\mathcal{S}}_{L,-}^{1}\left(\mathbf{V}_{G}\right) \cup \hat{\mathcal{V}}_{L,-}^{1}\left(\mathbf{V}_{G}\right) .
$$

We observe that

$$
\begin{gathered}
\hat{\mathcal{S}}_{R,+}^{3}\left(\mathbf{V}_{D}\right) \cup \hat{\mathcal{V}}_{R,+}^{3}\left(\mathbf{V}_{D}\right) \subset\left\{\mathbf{V} \in \Omega_{V} ; u>0\right\}, \\
\hat{\mathcal{S}}_{L,-}^{1}\left(\mathbf{V}_{G}\right) \cup \hat{\mathcal{V}}_{L,-}^{1}\left(\mathbf{V}_{G}\right) \subset\left\{\mathbf{V} \in \Omega_{V} ; u<0\right\}
\end{gathered}
$$

and therefore

$$
\left(\hat{\mathcal{S}}_{R,+}^{3}\left(\mathbf{V}_{D}\right) \cup \hat{\mathcal{V}}_{R,+}^{3}\left(\mathbf{V}_{D}\right)\right) \cap\left(\hat{\mathcal{S}}_{L,-}^{1}\left(\mathbf{V}_{G}\right) \cup \hat{\mathcal{V}}_{L,-}^{1}\left(\mathbf{V}_{G}\right)\right)=\emptyset
$$

Hence, besides the trivial case $\mathbf{V}_{G}=\mathbf{V}_{D}$ for which the solution $\mathbf{V}$ of the coupled Riemann problem is the constant state $\mathbf{V}_{G}=\mathbf{V}_{D}$, we have only to consider the cases corresponding to

$$
\mathbf{V}(0)=\mathbf{V}_{G} \in \hat{\mathcal{C}}_{R,+}^{3}\left(\mathbf{V}_{D}\right) \cup \hat{\mathcal{S}}_{R,+}^{3}\left(\mathbf{V}_{D}\right) \cup \hat{\mathcal{V}}_{R,+}^{3}\left(\mathbf{V}_{D}\right)
$$

or

$$
\mathbf{V}(0)=\mathbf{V}_{D} \in \hat{\mathcal{C}}_{L,-}^{1}\left(\mathbf{V}_{G}\right) \cup \hat{\mathcal{S}}_{L,-}^{1}\left(\mathbf{V}_{G}\right) \cup \hat{\mathcal{V}}_{L,-}^{1}\left(\mathbf{V}_{G}\right)
$$

and

$$
\mathbf{V}(0) \in \hat{\mathcal{C}}_{L,-}^{1}\left(\mathbf{V}_{G}\right) \cap\left(\hat{\mathcal{C}}_{R,+}^{3}\left(\mathbf{V}_{D}\right) \cup \hat{\mathcal{S}}_{R,+}^{3}\left(\mathbf{V}_{D}\right) \cup \hat{\mathcal{V}}_{R,+}^{3}\left(\mathbf{V}_{D}\right)\right) \neq \emptyset
$$

or

$$
\mathbf{V}(0) \in \hat{\mathcal{C}}_{R,+}^{3}\left(\mathbf{V}_{D}\right) \cap\left(\hat{\mathcal{C}}_{L,-}^{1}\left(\mathbf{V}_{G}\right) \cup \hat{\mathcal{S}}_{L,-}^{1}\left(\mathbf{V}_{G}\right) \cup \hat{\mathcal{V}}_{L,-}^{1}\left(\mathbf{V}_{G}\right)\right) \neq \emptyset
$$

In the case (82) the solution $\mathbf{V}$ of the coupled Riemann problem coincides with that of the $R$-Riemann problem $\mathbf{Z}_{R}\left(. ; \mathbf{V}_{G}, \mathbf{V}_{D}\right)$; i.e., the solution consists of constant states separated by $R$-waves with nonnegative speed:5 (see Fig. 4). Similarly, in the case (83), the solution $\mathbf{V}$ of the coupled Riemann problem coincides with that of the $L$-Riemann problem $\mathbf{Z}_{L}\left(. ; \mathbf{V}_{G}, \mathbf{V}_{D}\right)$; i.e., the solution consists of constant states separated by $L$-waves with nonpositive speeds.

On the other hand, the cases (84) and (85) correspond to a nontrivial coupled solution. Let us detail the various situations encountered in the case (84).

\footnotetext{
${ }^{5}$ In this section, we have to exclude stationary discontinuity waves. In particular, the 1 - $R$-wave must have here a positive speed if it is a shock!
} 


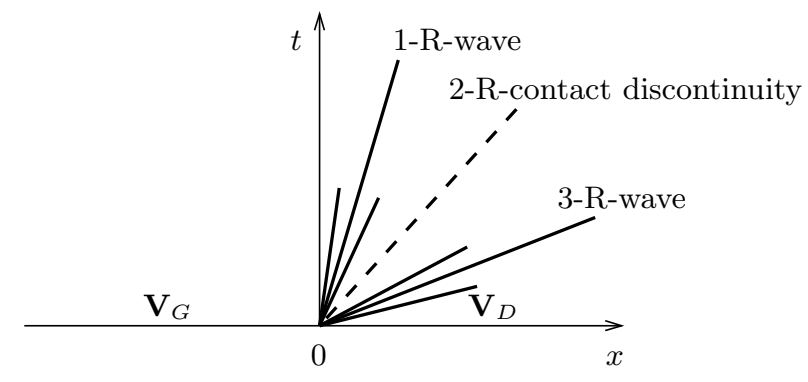

FIG. 4. The case (82).

(i) The case $\hat{\mathcal{C}}_{L,-}^{1}\left(\mathbf{V}_{G}\right) \cap \hat{\mathcal{C}}_{R,+}^{3}\left(\mathbf{V}_{D}\right) \neq \emptyset$.

The solution of the $\mathbf{V}$-coupled Riemann problem then consists of three constant states separated by a 1 - $L$-wave with a nonpositive speed and a 3 - $R$-wave with a nonnegative speed. Such a solution is necessarily unique since a 1 -wave curve and a 3 -wave curve can intersect at only one point (cf. Fig. 河).

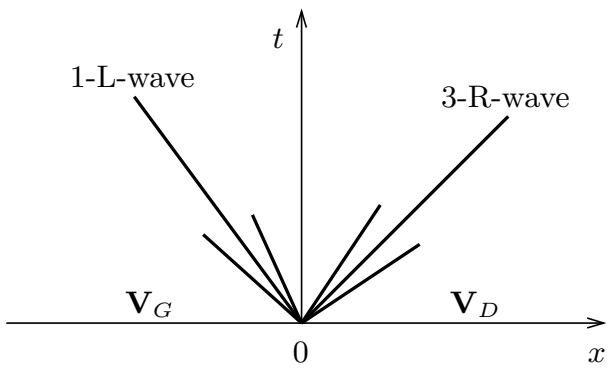

FIG. 5. The case $\hat{\mathcal{C}}_{L,-}^{1}\left(\mathbf{V}_{G}\right) \cap \hat{\mathcal{C}}_{R,+}^{3}\left(\mathbf{V}_{D}\right) \neq \emptyset$.

(ii) The case $\hat{\mathcal{C}}_{L,-}^{1}\left(\mathbf{V}_{G}\right) \cap \hat{\mathcal{S}}_{R,+}^{3}\left(\mathbf{V}_{D}\right) \neq \emptyset$.

Here the solution of the $\mathbf{V}$-coupled Riemann problem consists of four constant states separated by a 1 - $L$-wave with a nonpositive speed and a $2-R$-contact discontinuity and a 3- $R$-wave with positive speeds. Since the curves $\Gamma_{L}^{1}\left(\mathbf{V}_{G}\right)$ and $\Gamma_{R}^{3}\left(\mathbf{V}_{D}\right)$ are transverse at their intersection point, the curve $\hat{\mathcal{C}}_{L,-}^{1}\left(\mathbf{V}_{G}\right)$ is transverse to the surface $\hat{\mathcal{S}}_{R,+}^{3}\left(\mathbf{V}_{D}\right)$, which implies that such a solution is again necessarily unique (cf. Fig. 6).

(iii) The case $\hat{\mathcal{C}}_{L,-}^{1}\left(\mathbf{V}_{G}\right) \cap \hat{\mathcal{V}}_{R,+}^{3}\left(\mathbf{V}_{D}\right) \neq \emptyset$.

A solution of the $\mathbf{V}$-coupled Riemann problem consists here of five constant states separated by a $1-L$-wave with a nonpositive speed and a $1-R$-wave with a nonnegative speed followed by a 2 - $R$-contact discontinuity and a $3-R$-wave. Since the intersection of the curve $\hat{\mathcal{C}}_{L,-}^{1}\left(\mathbf{V}_{G}\right)$ and the volume $\hat{\mathcal{V}}_{R,+}^{3}\left(\mathbf{V}_{D}\right)$ is in general a part of the curve we find that such a solution is not unique. Indeed, we obtain a one-parameter family of solutions of the coupled Riemann problem (cf. Fig. 7).

Note that the case (85) leads to the symmetric situation.

To summarize, we observe that the solution of the coupled Riemann problem which is $\mathbf{V}$-continuous at the interface is not unique in general. On the one hand, the cases 


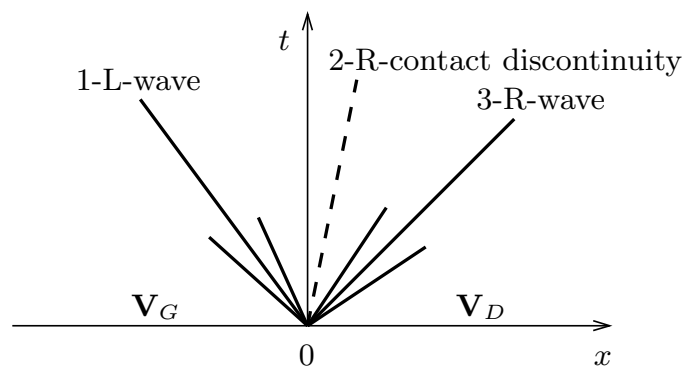

Fig. 6. The case $\hat{\mathcal{C}}_{L,-}^{1}\left(\mathbf{V}_{G}\right) \cap \hat{\mathcal{S}}_{R,+}^{3}\left(\mathbf{V}_{D}\right) \neq \emptyset$.

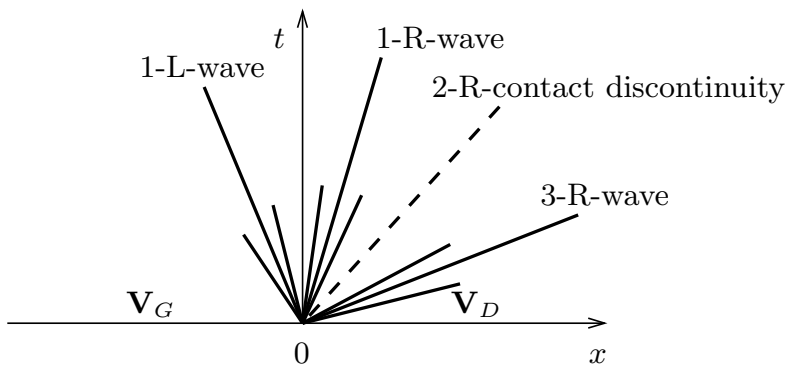

Fig. 7. The case $\hat{\mathcal{C}}_{L,-}^{1}\left(\mathbf{V}_{G}\right) \cap \hat{\mathcal{V}}_{R,+}^{3}\left(\mathbf{V}_{D}\right) \neq \emptyset$.

$\hat{\mathcal{C}}_{L,-}^{1}\left(\mathbf{V}_{G}\right) \cap \hat{\mathcal{V}}_{R,+}^{3}\left(\mathbf{V}_{D}\right) \neq \emptyset$ or $\hat{\mathcal{C}}_{R,+}^{3}\left(\mathbf{V}_{D}\right) \cap \hat{\mathcal{V}}_{L,-}^{1}\left(\mathbf{V}_{G}\right) \neq \emptyset$ lead to one-parameter families of solutions. On the other hand, for a given pair $\left(\mathbf{V}_{G}, \mathbf{V}_{D}\right)$, different cases may apply so that we obtain different types of solutions. Such a situation will indeed be encountered in Section 7 .

Nevertheless we can prove a simple and useful result of existence and uniqueness of subsonic $\mathbf{V}$-continuous solutions. By a subsonic solution, we mean a solution which satisfies

$$
|u(x, t)|< \begin{cases}c_{L}(x, t), & \text { if } x<0 \\ c_{R}(x, t), & \text { if } x>0 .\end{cases}
$$

Let us write the Euler system of gas dynamics equations in the nonconservative form

$$
\partial_{t} \mathbf{V}+\mathbf{B}_{\alpha}(\mathbf{V}) \partial_{x} \mathbf{V}=\mathbf{0}, \alpha=L, R
$$

Denoting by $\mathbf{r}_{\alpha}(\mathbf{V})$ the eigenvector of $\mathbf{B}_{\alpha}(\mathbf{V})$ associated with the eigenvalue $u$, we can now state

Theorem 3. Assume that we may choose $\mathbf{r}_{\alpha}(\mathbf{V})$ independently of $\alpha$. Then, given a pair $\left(\mathbf{V}_{G}, \mathbf{V}_{D}\right)$ such that

$$
\left|u_{G}\right|<c_{L, G}, \quad\left|u_{D}\right|<c_{R, D},
$$

there exists a unique subsonic $\mathbf{V}$-continuous solution of the corresponding $\mathbf{V}$-coupled Riemann problem. 
Proof. Let $\mathbf{Z}(x, t)$ be a subsonic $\mathbf{V}$-continuous solution; we observe that $\mathbf{Z}$ cannot have a 1-wave with a nonnegative speed or a 3 -wave with a nonpositive speed. This is obvious if the wave is a rarefaction and again it follows from the Lax entropy condition if the wave is a shock. Now, using the above results, we know that the solution $\mathbf{Z}$ is necessarily of one of the forms corresponding to the cases (i) and (ii) or to the symmetric cases. Hence we have only two classes of possible solutions. Each of them consists of four constant states separated by a $1-L$-wave (with a nonpositive speed), a 2 -contact discontinuity which can be an $L$-wave (solution 1 ) or an $R$-wave (solution 2) and a 3 - $R$-wave (with a nonnegative speed); some of these waves may not exist (cf. Fig. 8).

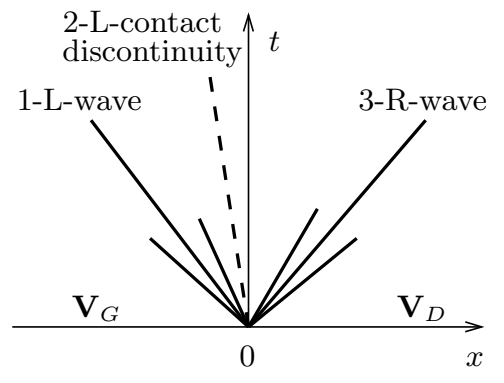

Solution 1

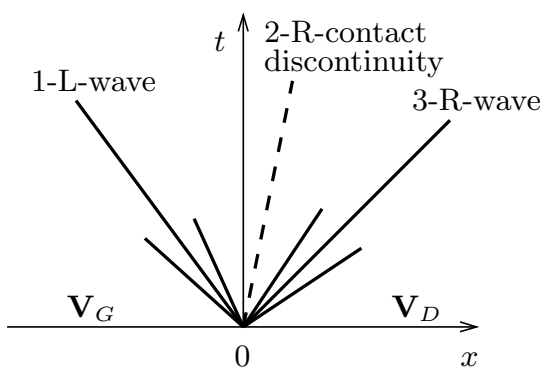

Solution 2

FIG. 8. Two possible solutions.

We now prove that, under the hypotheses of the theorem, there exists among these two classes a unique solution of the coupled Riemann problem. Let

$$
\mathbf{V}=\Psi_{\alpha}^{i}\left(\varepsilon ; \mathbf{V}_{0}\right)
$$

be a parametrization of the wave curve $\mathcal{C}_{\alpha}^{i}\left(\mathbf{V}_{0}\right)$; then a solution of class 1 is given by

$$
\mathbf{V}_{D}=\Psi_{R}^{3}\left(\varepsilon_{3}^{1} ; \Psi_{L}^{2}\left(\varepsilon_{2}^{1} ; \Psi_{L}^{1}\left(\varepsilon_{1}^{1} ; \mathbf{V}_{G}\right)\right)\right)
$$

while a solution of class 2 is obtained through

$$
\mathbf{V}_{D}=\Psi_{R}^{3}\left(\varepsilon_{3}^{2} ; \Psi_{R}^{2}\left(\varepsilon_{2}^{2} ; \Psi_{L}^{1}\left(\varepsilon_{1}^{2} ; \mathbf{V}_{G}\right)\right)\right) .
$$

The triple $\left(\varepsilon_{1}^{k}, \varepsilon_{2}^{k}, \varepsilon_{3}^{k}\right)$ characterizes a solution of class $k, k=1,2$. Next we notice that the wave curve $\mathcal{C}_{\alpha}^{2}\left(\mathbf{V}_{0}\right)$ may be defined by

$$
\frac{d \Psi_{\alpha}^{2}}{d \varepsilon}=\mathbf{r}_{\alpha}\left(\Psi_{\alpha}^{2}\right), \quad \varepsilon=u-u_{0} .
$$

If we assume that $\mathbf{r}_{L}=\mathbf{r}_{R}$, we obtain

$$
\Psi_{L}^{2}\left(\varepsilon ; \mathbf{V}_{0}\right)=\Psi_{R}^{2}\left(\varepsilon ; \mathbf{V}_{0}\right)=\Psi^{2}\left(\varepsilon ; \mathbf{V}_{0}\right)
$$

so that we have to look for a solution given by

$$
\mathbf{V}_{D}=\Psi_{R}^{3}\left(\varepsilon_{3} ; \Psi^{2}\left(\varepsilon_{2} ; \Psi_{L}^{1}\left(\varepsilon_{1} ; \mathbf{V}_{G}\right)\right)\right) .
$$

Clearly there exists a unique triple $\left(\varepsilon_{1}, \varepsilon_{2}, \varepsilon_{3}\right)$ solution of (89). On the other hand, it follows from the hypothesis (88) that the 1 - $L$-wave has a nonpositive speed while the 
3 - $R$-wave has a nonnegative speed so that the constructed solution is indeed a solution of the $\mathbf{V}$-coupled Riemann problem and is subsonic.

It remains to check whether we can choose $\mathbf{r}_{\alpha}(\mathbf{V})$ independently of $\alpha$ or not. In the case where $\mathbf{V}=(\rho, u, p)^{T}$, we have

$$
\mathbf{r}(\mathbf{V})=(1,0,0)^{T},
$$

and Theorem 3 applies independently of the equation of state. However, this is no longer true if $\mathbf{V}=(\rho, \rho u, \rho e)^{T}$ or $\mathbf{V}=(\rho, u, h)^{T}$. Being more specific, we asume that each equation of state is of Grüneisen type, i.e.,

$$
p=(\gamma-1) \rho \varepsilon+c_{r e f}^{2}\left(\rho-\rho_{\text {ref }}\right) .
$$

Then it is a simple matter to check that we have for $\mathbf{V}=(\rho, \rho u, \rho e)^{T}$,

$$
\mathbf{r}(\mathbf{V})=\left(1, u, \frac{u^{2}}{2}-\frac{c_{r e f}^{2}}{\gamma-1}\right)^{T}
$$

and for $\mathbf{V}=(\rho, u, h)^{T}$,

$$
\mathbf{r}(\mathbf{V})=\left(1,0, \frac{\rho}{h+c_{r e f}^{2} /(\gamma-1)}\right)^{T} .
$$

In these two latter cases, we find that $\mathbf{r}(\mathbf{V})$ depends only on $c_{r e f}^{2} /(\gamma-1)$ so that Theorem 3 applies in particular for polytropic equations of state but not for general Grüneisen ones.

As a final remark, it is worthwile to notice that, expressed in the variables $\mathbf{U}$, all the previous solutions present in general an additional stationary discontinuity at $x=0$.

5. The V-coupling conditions. We pass to the construction of discontinuous solutions of the $\mathbf{V}$-coupled Riemann problem. We begin with the stationary solutions. Clearly the function

$$
\mathbf{U}(x, t)=\left\{\begin{array}{ll}
\mathbf{U}_{G}, & \text { if } x<0, \\
\mathbf{U}_{D}, & \text { if } x>0,
\end{array} t>0\right.
$$

is a stationary solution of the $\mathbf{V}$-coupled Riemann problem if and only if the pair $\left(\mathbf{V}_{G}, \mathbf{V}_{D}\right)$ defined by (80) satisfies the $\mathbf{V}$-coupling conditions

$$
\left\{\begin{array}{l}
\mathbf{V}_{G} \in \mathcal{O}_{L}\left(\mathbf{V}_{D}\right) \\
\mathbf{V}_{D} \in \mathcal{O}_{R}\left(\mathbf{V}_{G}\right)
\end{array}\right.
$$

As a first remark, we note that each of the above coupling conditions (90) can be expressed in a fairly simple form, which will be used constantly later on.

Lemma 3. We have $\mathbf{V}_{G} \in \mathcal{O}_{L}\left(\mathbf{V}_{D}\right)$ if and only if

$$
\mathbf{V}_{G}=\mathbf{Z}_{L}\left(0-; \mathbf{V}_{G}, \mathbf{V}_{D}\right)
$$

and similarly we have $\mathbf{V}_{D} \in \mathcal{O}_{R}\left(\mathbf{V}_{G}\right)$ if and only if

$$
\mathbf{V}_{D}=\mathbf{Z}_{R}\left(0+; \mathbf{V}_{G}, \mathbf{V}_{D}\right) \text {. }
$$


Proof. We prove only the first assertion. Recall that

$$
\mathcal{O}_{L}\left(\mathbf{V}_{D}\right)=\left\{\mathbf{Z}_{L}\left(0-; \mathbf{V}, \mathbf{V}_{G}\right) ; \mathbf{V} \in \Omega_{V}\right\} .
$$

Hence the if part of the result is obvious. Conversely, if $\mathbf{V}_{G}=\mathbf{Z}_{L}\left(0-; \mathbf{V}, \mathbf{V}_{D}\right)$ for some $\mathbf{V} \in \Omega_{V}$, the function defined by

$$
\begin{cases}\mathbf{V}_{G}, & \text { if } x / t<0 \\ \mathbf{V}_{G}=\mathbf{Z}_{L}\left(x / t ; \mathbf{V}, \mathbf{V}_{D}\right), & \text { if } x / t \geq 0\end{cases}
$$

is clearly the solution $\mathbf{Z}_{L}\left(x / t ; \mathbf{V}_{G}, \mathbf{V}_{D}\right)$ of the $L$-Riemann problem associated with the pair $\left(\mathbf{V}_{G}, \mathbf{V}_{D}\right)$ so that $\mathbf{V}_{G}=\mathbf{Z}_{L}\left(0-; \mathbf{V}_{G}, \mathbf{V}_{D}\right)$.

Now, using (67) and Theorem 1) the conditions (90) read:

$$
\begin{aligned}
& \mathbf{V}_{G} \in\left\{\mathbf{V}_{D}\right\} \cup \mathcal{C}_{L,+}^{3}\left(\mathbf{V}_{D}\right) \cup \mathcal{S}_{L,+}^{3}\left(\mathbf{V}_{D}\right) \cup \mathcal{V}_{L,+}^{3}\left(\mathbf{V}_{D}\right), \\
& \mathbf{V}_{D} \in\left\{\mathbf{V}_{G}\right\} \cup \mathcal{C}_{R,-}^{1}\left(\mathbf{V}_{G}\right) \cup \mathcal{S}_{R,-}^{1}\left(\mathbf{V}_{G}\right) \cup \mathcal{V}_{R,-}^{1}\left(\mathbf{V}_{G}\right) .
\end{aligned}
$$

If we exclude the trivial case $\mathbf{V}_{G}=\mathbf{V}_{D}$, we have only to consider the following situations:

$$
\begin{gathered}
\mathbf{V}_{G} \in \mathcal{C}_{L,+}^{3}\left(\mathbf{V}_{D}\right), \mathbf{V}_{D} \in \mathcal{C}_{R,-}^{1}\left(\mathbf{V}_{G}\right), \\
\mathbf{V}_{G} \in \mathcal{C}_{L,+}^{3}\left(\mathbf{V}_{D}\right), \mathbf{V}_{D} \in \mathcal{S}_{R,-}^{1}\left(\mathbf{V}_{G}\right) \text { or } \mathbf{V}_{G} \in \mathcal{S}_{L,+}^{3}\left(\mathbf{V}_{D}\right), \mathbf{V}_{D} \in \mathcal{C}_{R,-}^{1}\left(\mathbf{V}_{G}\right), \\
\mathbf{V}_{G} \in \mathcal{S}_{L,+}^{3}\left(\mathbf{V}_{D}\right), \mathbf{V}_{D} \in \mathcal{S}_{R,-}^{1}\left(\mathbf{V}_{G}\right), \\
\mathbf{V}_{G} \in \mathcal{C}_{L,+}^{3}\left(\mathbf{V}_{D}\right), \mathbf{V}_{D} \in \mathcal{V}_{R,-}^{1}\left(\mathbf{V}_{G}\right) \text { or } \mathbf{V}_{G} \in \mathcal{V}_{L,+}^{3}\left(\mathbf{V}_{D}\right), \mathbf{V}_{D} \in \mathcal{C}_{R,-}^{1}\left(\mathbf{V}_{G}\right), \\
\mathbf{V}_{G} \in \mathcal{S}_{L,+}^{3}\left(\mathbf{V}_{D}\right), \mathbf{V}_{D} \in \mathcal{V}_{R,-}^{1}\left(\mathbf{V}_{G}\right) \text { or } \mathbf{V}_{G} \in \mathcal{V}_{L,+}^{3}\left(\mathbf{V}_{D}\right), \mathbf{V}_{D} \in \mathcal{S}_{R,-}^{1}\left(\mathbf{V}_{G}\right), \\
\mathbf{V}_{G} \in \mathcal{V}_{L,+}^{3}\left(\mathbf{V}_{D}\right), \mathbf{V}_{D} \in \mathcal{V}_{R,-}^{1}\left(\mathbf{V}_{G}\right) .
\end{gathered}
$$

Consider first the situation (93). It implies that the wave curves $\mathcal{C}_{L,+}^{3}\left(\mathbf{V}_{D}\right)$ and $\mathcal{C}_{R,-}^{1}\left(\mathbf{V}_{G}\right)$ intersect and the states $\mathbf{V}_{G}$ and $\mathbf{V}_{D}$ belong to the intersection. Indeed such wave curves can intersect at one point at most which yields $\mathbf{V}_{G}=\mathbf{V}_{D}$ : we find again the trivial case.

In order to study the situations (94)- (98), we need to specify the variables V. We first consider the case where

$$
\mathbf{V}=(\rho, u, p)^{T} .
$$

Let us first analyze the situation (94), where for instance $\mathbf{V}_{G} \in \mathcal{C}_{L,+}^{3}\left(\mathbf{V}_{D}\right), \mathbf{V}_{D} \in$ $\mathcal{S}_{R,-}^{1}\left(\mathbf{V}_{G}\right)$. Since $u$ and $p$ are components of $\mathbf{V}$, we may project onto the $(u, p)$-plane to obtain

$$
\begin{gathered}
\Upsilon_{G}=\left(u_{G}, p_{G}\right) \in\left\{\Upsilon \in \Gamma_{L}^{3}\left(\mathbf{V}_{D}\right) ; u \geq u_{L, \min }^{3}\left(\mathbf{V}_{D}\right)\right\}, \\
\Upsilon_{D}=\left(u_{D}, p_{D}\right) \in\left\{\Upsilon \in \Gamma_{R}^{1}\left(\mathbf{V}_{G}\right) ; u \leq 0\right\} .
\end{gathered}
$$

Again two such curves $\Gamma_{L}^{3}\left(\mathbf{V}_{D}\right)$ and $\Gamma_{R}^{1}\left(\mathbf{V}_{G}\right)$ of the $(u, p)$-plane can intersect at only one point which yields

$$
u_{G}=u_{D}, p_{G}=p_{D}
$$

In addition, since $\mathbf{V}_{G} \in \mathcal{C}_{L}^{3}\left(\mathbf{V}_{D}\right)$, we find

$$
\rho_{G}=\varphi_{L, D}\left(p_{G}\right)=\varphi_{L, D}\left(p_{D}\right)=\rho_{D}
$$


and therefore $\mathbf{V}_{G}=\mathbf{V}_{D}$. The same conclusion clearly holds when $\mathbf{V}_{G} \in \mathcal{S}_{L,+}^{3}\left(\mathbf{V}_{D}\right), \mathbf{V}_{D} \in$ $\mathcal{C}_{R,-}^{1}\left(\mathbf{V}_{G}\right)$. Hence (94) reduces to the trivial case.

We pass to the situation (95). By projection onto the $(u, p)$-space, we find here

$$
\Upsilon_{G} \in\left\{\Upsilon \in \Gamma_{L}^{3}\left(\mathbf{V}_{D}\right) ; u \geq 0\right\}
$$

and

which yields

$$
\Upsilon_{D} \in\left\{\Upsilon \in \Gamma_{R}^{1}\left(\mathbf{V}_{G}\right) ; u \leq 0\right\}
$$

$$
u_{G}=u_{D}=0, p_{G}=p_{D}
$$

Since $\rho_{G}$ and $\rho_{D}$ are arbitrary, the situation (95) corresponds to a stationary contact discontinuity.

The situations (96), (97) and (98) are addressed in the next result: using the characterizations of the sets $\mathcal{C}_{L,+}^{3}\left(\mathbf{V}_{D}\right), \mathcal{S}_{L,+}^{3}\left(\mathbf{V}_{D}\right), \mathcal{V}_{L,+}^{3}\left(\mathbf{V}_{D}\right)$ and $\mathcal{C}_{R,-}^{1}\left(\mathbf{V}_{G}\right), \mathcal{S}_{R,-}^{1}\left(\mathbf{V}_{G}\right)$, $\mathcal{V}_{R,-}^{1}\left(\mathbf{V}_{G}\right)$, we can easily prove

Theorem 4. Assume the hypothesis (99). The coupling constraints (90) hold if and only if the pair $\left(\mathbf{V}_{G}, \mathbf{V}_{D}\right)$ satisfies one of the following five conditions:

(i) $\mathbf{V}_{G}=\mathbf{V}_{D}$ (the trivial case);

(ii) $u_{G}=u_{D}=0, p_{G}=p_{D}$ (stationary contact discontinuity);

(iii) we have on the one hand $\left(\mathbf{V}_{G} \in \mathcal{C}_{L,+}^{3}\left(\mathbf{V}_{D}\right) \cup \mathcal{S}_{L,+}^{3}\left(\mathbf{V}_{D}\right)\right)$

$$
u_{G}=u_{D}+\Phi_{L, D}\left(p_{G}\right)
$$

with either

$$
u_{L, \min }^{3}\left(\mathbf{V}_{D}\right) \leq u_{G}<0, \rho_{G}=\varphi_{L, D}\left(p_{G}\right)
$$

or

$$
u_{G} \geq 0, \rho_{G}>0 \text { arbitrary }
$$

and on the other hand $\left(\mathbf{V}_{D} \in \mathcal{V}_{R,-}^{1}\left(\mathbf{V}_{G}\right)\right)$

$$
u_{D} \leq-c_{R, D}, u_{R, s}^{3}\left(\mathbf{V}_{D}\right) \geq u_{G}-\Phi_{R, G}\left(p_{R, s}^{3}\left(\mathbf{V}_{D}\right)\right) ;
$$

(iv) (the symmetric situation) we have on the one hand

$$
u_{D}=u_{G}-\Phi_{R, G}\left(p_{D}\right)
$$

with either $\left(\mathbf{V}_{D} \in \mathcal{C}_{R,-}^{1}\left(\mathbf{V}_{G}\right) \cup \mathcal{S}_{R,-}^{1}\left(\mathbf{V}_{G}\right)\right)$

$$
0<u_{D} \leq u_{R, \max }^{1}\left(\mathbf{V}_{G}\right), \rho_{D}=\varphi_{R, G}\left(p_{D}\right)
$$

or

$$
u_{D} \leq 0, \rho_{D}>0 \text { arbitrary }
$$

and on the other hand $\left(\mathbf{V}_{G} \in \mathcal{V}_{L,+}^{3}\left(\mathbf{V}_{D}\right)\right)$

$$
u_{G} \geq c_{R, G}, u_{L, s}^{1}\left(\mathbf{V}_{G}\right) \leq u_{D}+\Phi_{L, D}\left(p_{L, s}^{1}\left(\mathbf{V}_{G}\right)\right) ;
$$

(v) we have $\left(\mathbf{V}_{D} \in \mathcal{V}_{R,-}^{1}\left(\mathbf{V}_{G}\right), \mathbf{V}_{G} \in \mathcal{V}_{L,+}^{3}\left(\mathbf{V}_{D}\right)\right)$

$$
\begin{cases}u_{D} \leq-c_{R, D}, & u_{R, s}^{3}\left(\mathbf{V}_{D}\right) \geq u_{G}-\Phi_{R, G}\left(p_{R, s}^{3}\left(\mathbf{V}_{D}\right)\right), \\ u_{G} \geq c_{R, G}, & u_{L, s}^{1}\left(\mathbf{V}_{G}\right) \leq u_{D}+\Phi_{L, D}\left(p_{L, s}^{1}\left(\mathbf{V}_{G}\right)\right) .\end{cases}
$$


REMARK 4. In the case of the polytropic equations of state

$$
p_{\alpha}(\rho, \varepsilon)=\left(\gamma_{\alpha}-1\right) \rho \varepsilon, \quad \alpha=L, R,
$$

we may apply Propositions 7 and 9 , using the obvious notation, the conditions $\mathbf{V}_{D} \in$ $\mathcal{C}_{R,-}^{1}\left(\mathbf{V}_{G}\right)$ and $\mathbf{V}_{G} \in \mathcal{V}_{L,+}^{3}\left(\mathbf{V}_{D}\right)$ take respectively the simple forms

$$
u_{D}<0, \rho_{D} \geq \mathcal{R}_{R, G}^{-}\left(u_{D}, p_{D}\right)
$$

and

$$
u_{G}>0, \rho_{G} \geq \mathcal{R}_{L, D}^{+}\left(u_{G}, p_{G}\right) .
$$

Let us now briefly discuss the case of a more general choice of variables $\mathbf{V}$. If $p$ is not a transmitted variable at the interface, i.e., $p$ is not a variable of $\mathbf{V}$, the projection onto the $(u, p)$-plane is no longer suitable. Indeed, consider for instance the sitution (94) and more specifically the case

$$
\mathbf{V}_{G} \in \mathcal{C}_{L,+}^{3}\left(\mathbf{V}_{D}\right), \mathbf{V}_{D} \in \mathcal{S}_{R,-}^{1}\left(\mathbf{V}_{G}\right)
$$

By projection onto the $(u, p)$-plane, we obtain in particular

$$
\left(u_{G}, p_{L}\left(\mathbf{V}_{G}\right)\right) \in \Gamma_{L}^{3}\left(\mathbf{V}_{D}\right),\left(u_{D}, p_{R}\left(\mathbf{V}_{D}\right)\right) \in \Gamma_{R}^{1}\left(\mathbf{V}_{G}\right) .
$$

But now, the curve $\Gamma_{R}^{1}\left(\mathbf{V}_{G}\right)$ is issued from the point $\left(u_{G}, p_{R}\left(\mathbf{V}_{G}\right)\right)$ while $\Gamma_{L}^{3}\left(\mathbf{V}_{D}\right)$ is issued from $\left(u_{D}, p_{L}\left(\mathbf{V}_{D}\right)\right)$ and the properties (102) do not imply at least in general $p_{L}\left(\mathbf{V}_{G}\right)=p_{R}\left(\mathbf{V}_{D}\right)$. In fact, in order to obtain simple results, we are led as in $\mathrm{ACC}^{+} 06 \mathrm{c}$ ] to restrict ourselves to the case where the equations of state are of the form

$$
p_{\alpha}(\rho, \varepsilon)=\chi_{\alpha}(\pi(\mathbf{V})), \alpha=L, R
$$

for some thermodynamic variable $\pi(\mathbf{V})$ independent of the pressure laws. We will assume for simplicity that $\pi(\mathbf{V})$ is a positive function and, for any $\alpha=L, R, \chi_{\alpha}$ is a strictly increasing function from $\mathbb{R}_{+}$onto itself. As a consequence, we can invert each mapping $\pi \mapsto p_{\alpha}=\chi_{\alpha}(\pi)$, and moreover $\pi$ and $p_{\alpha}$ decrease or increase simultaneously.

For the choices $\mathbf{V}=\mathbf{U}=(\rho, \rho u, \rho e)^{T}$ and $\mathbf{V}=(\rho, u, h)^{T}$ that we have in mind, this property (103) holds for the polytropic equations of state (100). Indeed, we can take in the first case,

$$
\pi(\mathbf{V})=\rho \varepsilon=\rho\left(e-\frac{1}{2} u^{2}\right), \chi_{\alpha}(\pi)=\left(\gamma_{\alpha}-1\right) \pi
$$

and in the second case,

$$
\pi(\mathbf{V})=\rho h, \chi_{\alpha}(\pi)=\frac{\gamma_{\alpha}-1}{\gamma_{\alpha}} \pi
$$

Note however that this is not true of equations of state of Grüneisen type.

Now, if (103) holds, we can project the coupling constraints onto the $(u, \pi)$-plane. If we set for any state $\mathbf{V}_{0}$ and any $\alpha=L, R$,

$$
\tilde{\Phi}_{\alpha, 0}(\pi)=\Phi_{\alpha, 0}\left(\chi_{\alpha}(\pi)\right), \tilde{\varphi}_{\alpha, 0}(\pi)=\varphi_{\alpha, 0}\left(\chi_{\alpha}(\pi)\right),
$$

we obtain a parametric representation of the curve $\mathcal{C}_{\alpha}^{1}\left(\mathbf{V}_{0}\right)\left(\operatorname{resp} . \mathcal{C}_{\alpha}^{3}\left(\mathbf{V}_{0}\right)\right)$

$$
\left\{\begin{array}{l}
u=u_{0}-\tilde{\Phi}_{\alpha, 0}(\pi) \quad\left(\text { resp. } u=u_{0}+\tilde{\Phi}_{\alpha, 0}(\pi)\right), \\
\rho=\tilde{\varphi}_{\alpha, 0}(\pi), p=\chi_{\alpha}(\pi)
\end{array}\right.
$$


so that the projection $\tilde{\Gamma}_{\alpha}^{1}\left(\mathbf{V}_{0}\right)\left(\operatorname{resp} . \tilde{\Gamma}_{\alpha}^{3}\left(\mathbf{V}_{0}\right)\right)$ of $\mathcal{C}_{\alpha}^{1}\left(\mathbf{V}_{0}\right)\left(\right.$ resp. $\left.\mathcal{C}_{\alpha}^{3}\left(\mathbf{V}_{0}\right)\right)$ is obtained as

$$
\tilde{\Gamma}_{\alpha}^{1}\left(\mathbf{V}_{0}\right)=\left\{(u, \pi) ; u=u_{0}-\tilde{\Phi}_{\alpha, 0}(\pi)\right\}
$$

(resp. as

$$
\left.\tilde{\Gamma}_{\alpha}^{3}\left(\mathbf{V}_{0}\right)=\left\{(u, \pi) ; u=u_{0}+\tilde{\Phi}_{\alpha, 0}(\pi)\right\}\right) .
$$

From now on, we assume the hypothesis (103). Consider again the situation (101). By projection onto the $(u, \pi)$-plane, we obtain

$$
\left(u_{G}, \pi_{G}\right) \in \tilde{\Gamma}_{L}^{3}\left(\mathbf{V}_{D}\right),\left(u_{D}, \pi_{D}\right) \in \tilde{\Gamma}_{R}^{1}\left(\mathbf{V}_{G}\right) .
$$

Clearly the curves $\tilde{\Gamma}_{L}^{3}\left(\mathbf{V}_{D}\right)$ and $\tilde{\Gamma}_{R}^{1}\left(\mathbf{V}_{G}\right)$ intersect at one point at most so that (107) yields

$$
u_{G}=u_{D}, \pi_{G}=\pi_{D}
$$

and, in addition,

$$
\rho_{G}=\tilde{\varphi}_{L, D}\left(\pi_{G}\right)=\tilde{\varphi}_{L, D}\left(\pi_{D}\right)=\rho_{D} .
$$

Here again, the situation (101) (and also (94)) reduces to the trivial case.

If we turn to the situation (95), we now obtain

$$
\left\{\begin{array}{l}
\left(u_{G}, \pi_{G}\right) \in \tilde{\Gamma}_{L}^{3}\left(\mathbf{V}_{D}\right), u_{G} \geq 0 \\
\left(u_{D}, \pi_{D}\right) \in \tilde{\Gamma}_{R}^{1}\left(\mathbf{V}_{G}\right), u_{D} \leq 0
\end{array}\right.
$$

which yields

$$
u_{G}=u_{D}=0, \pi_{G}=\pi_{D}
$$

In other words, we get the continuity of the velocity $u$ and of the thermodynamic variable $\pi$ at the interface but not the continuity of the pressure $p$. In fact, we obtain only

$$
\chi_{L}^{-1}\left(p_{G}\right)=\chi_{R}^{-1}\left(p_{D}\right),
$$

which gives for polytropic gases,

$$
\frac{p_{G}}{\gamma_{L}-1}=\frac{p_{D}}{\gamma_{R}-1} \text { if } \mathbf{V}=(\rho, \rho u, \rho e)^{T}
$$

and

$$
\frac{\gamma_{L}}{\gamma_{L}-1} p_{G}=\frac{\gamma_{R}}{\gamma_{R}-1} p_{D} \text { if } \mathbf{V}=(\rho, u, h)^{T} .
$$

On the other hand the conditions (iii), (iv) and (v) of Theorem 4 remain unchanged.

6. Solutions of the coupled Riemann problem which are V-discontinuous at the interface. We now look for nonstationary solutions $\mathbf{V}=\mathbf{V}(x / t)$ of the $\mathbf{V}$-coupled Riemann problem which are discontinuous at the interface $x=0$. For simplicity, we set

$$
\mathbf{V}_{ \pm}=\mathbf{V}\left(0_{ \pm}\right)
$$

Such solutions are characterized by the following properties (see also Fig. 9 and Fig. 10): (i) the pair $\left(\mathbf{V}_{-}, \mathbf{V}_{+}\right)$satisfies the coupling constraints

$$
\left\{\begin{array}{l}
\mathbf{V}_{-} \in \mathcal{O}_{L}\left(\mathbf{V}_{+}\right) \\
\mathbf{V}_{+} \in \mathcal{O}_{R}\left(\mathbf{V}_{-}\right)
\end{array}\right.
$$


(ii) the waves of the solution $\mathbf{Z}_{L}\left(. ; \mathbf{V}_{G}, \mathbf{V}_{-}\right)$of the $L$-Riemann problem associated with the initial pair $\left(\mathbf{V}_{G}, \mathbf{V}_{-}\right)$have nonpositive velocities;

(iii) the waves of the solution $\mathbf{Z}_{R}\left(. ; \mathbf{V}_{+}, \mathbf{V}_{D}\right)$ of the $R$-Riemann problem associated with the initial pair $\left(\mathbf{V}_{+}, \mathbf{V}_{D}\right)$ have nonnegative velocities.

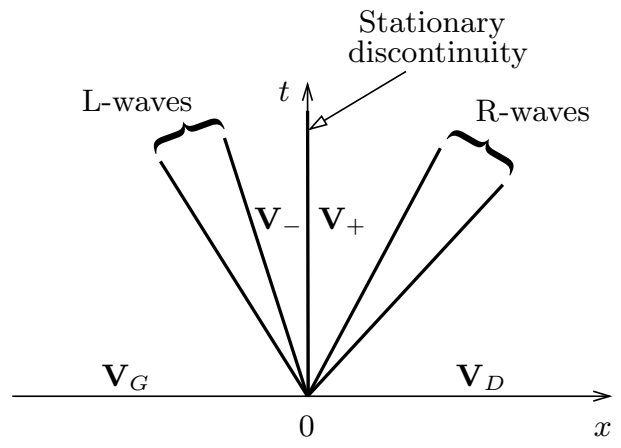

FIG. 9. Generic form of $\mathbf{V}$-discontinuous solutions of the coupled Riemann problem.

We want to find all the possible forms of these discontinuous solutions. We begin with Lemma 4. We have

$$
\left\{\begin{array}{l}
\mathbf{V}_{-}=\mathbf{Z}_{L}\left(0_{-} ; \mathbf{V}_{G}, \mathbf{V}_{+}\right) \\
\mathbf{V}_{+}=\mathbf{Z}_{R}\left(0_{+} ; \mathbf{V}_{-}, \mathbf{V}_{D}\right)
\end{array}\right.
$$

Proof. Since

$$
\mathcal{O}_{L}\left(\mathbf{V}_{+}\right)=\left\{\mathbf{Z}_{L}\left(0_{-} ; \mathbf{V}, \mathbf{V}_{+}\right) ; \mathbf{V} \in \Omega_{V}\right\},
$$

the property $\mathbf{V}_{-} \in \mathcal{O}_{L}\left(\mathbf{V}_{+}\right)$exactly means that the waves of $\mathbf{Z}_{L}\left(. ; \mathbf{V}_{-}, \mathbf{V}_{+}\right)$have nonnegative speeds; the first assertion (109) follows from the first coupling constraint (108) and the property (ii). The second assertion (109) follows from the second coupling constraint (108) and the property (iii).

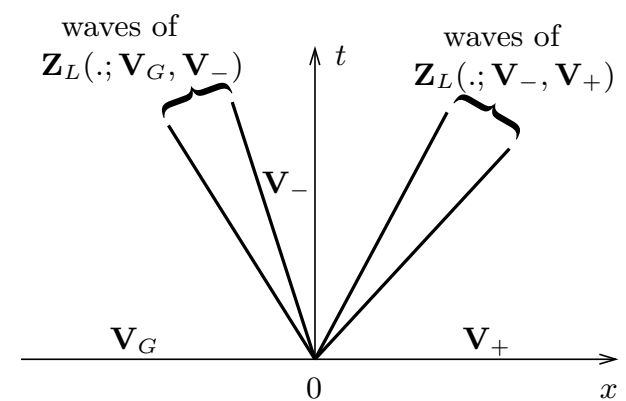

$\mathrm{L}$

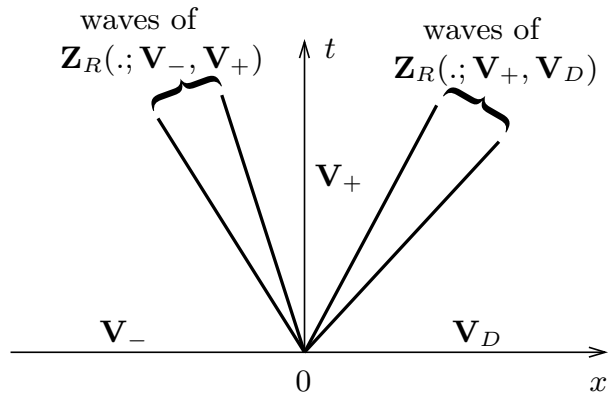

$\mathrm{R}$

FIG. 10. Generic forms of $\mathbf{Z}_{L}\left(. ; \mathbf{V}_{G}, \mathbf{V}_{+}\right)$and $\mathbf{Z}_{R}\left(. ; \mathbf{V}_{-}, \mathbf{V}_{D}\right)$.

Let us then give some easy consequences of the previous lemma. 
Lemma 5. Assume that $\mathbf{Z}_{L}\left(. ; \mathbf{V}_{-}, \mathbf{V}_{+}\right)$possesses a 1-wave. Then:

(i) if $\lambda_{L}^{1}\left(\mathbf{V}_{G}\right)=u_{G}-c_{L, G} \geq 0$, we have $\mathbf{V}_{-}=\mathbf{V}_{G}$;

(ii) if $\lambda_{L}^{1}\left(\mathbf{V}_{G}\right)<0, \mathbf{Z}_{L}\left(. ; \mathbf{V}_{G}, \mathbf{V}_{-}\right)$has a 1-rarefaction wave and $\mathbf{V}_{-}$is a sonic state of this 1-wave.

Proof. If $\lambda_{L}^{1}\left(\mathbf{V}_{G}\right) \geq 0, \mathbf{Z}_{L}\left(. ; \mathbf{V}_{G}, \mathbf{V}_{-}\right)$is a constant function (cf. Fig. 10) and we have $\mathbf{V}_{-}=\mathbf{V}_{G}$. On the other hand, if $\lambda_{L}^{1}\left(\mathbf{V}_{G}\right)<0, \mathbf{Z}_{L}\left(. ; \mathbf{V}_{G}, \mathbf{V}_{-}\right)$possesses a 1-wave whose speed is nonpositive. This 1-wave must fit the 1-wave of $\mathbf{Z}_{L}\left(. ; \mathbf{V}_{-}, \mathbf{V}_{+}\right)$whose speed is nonnegative in order to form the 1-wave of $\mathbf{Z}_{L}\left(. ; \mathbf{V}_{G}, \mathbf{V}_{+}\right)$. Hence this 1-wave is a rarefaction and $\mathbf{V}_{-}$is the sonic state of this rarefaction wave.

Similarly, we can state

Lemma 6. Assume that $\mathbf{Z}_{R}\left(. ; \mathbf{V}_{-}, \mathbf{V}_{+}\right)$possesses a 3-wave. Then:

(i) if $\lambda_{R}^{3}\left(\mathbf{V}_{D}\right)=u_{D}+c_{R, D} \leq 0$, we have $\mathbf{V}_{+}=\mathbf{V}_{D}$;

(ii) if $\lambda_{R}^{3}\left(\mathbf{V}_{D}\right)>0, \mathbf{Z}_{R}\left(. ; \mathbf{V}_{+}, \mathbf{V}_{D}\right)$ has a 3 -rarefaction wave and $\mathbf{V}_{+}$is a sonic state of this 3 -wave.

Let us detail the four possible cases.

6.1. $\boldsymbol{Z}_{L}\left(. ; \boldsymbol{V}_{-}, \boldsymbol{V}_{+}\right)$has a 1 -wave and $\boldsymbol{Z}_{R}\left(. ; \boldsymbol{V}_{-}, \boldsymbol{V}_{+}\right)$has a 3 -wave. We obtain here

$$
\mathbf{V}_{-}= \begin{cases}\mathbf{V}_{G}, & \text { if } \lambda_{L}^{1}\left(\mathbf{V}_{G}\right) \geq 0 \\ \text { the sonic state of } \mathcal{C}_{L, r}^{1}\left(\mathbf{V}_{G}\right), & \text { if } \lambda_{L}^{1}\left(\mathbf{V}_{G}\right)<0\end{cases}
$$

and

$$
\mathbf{V}_{+}= \begin{cases}\mathbf{V}_{D}, & \text { if } \lambda_{R}^{3}\left(\mathbf{V}_{D}\right) \leq 0 \\ \text { the sonic state of } \mathcal{C}_{R, r}^{3}\left(\mathbf{V}_{D}\right), & \text { if } \lambda_{R}^{3}\left(\mathbf{V}_{D}\right)>0\end{cases}
$$

Note that $\mathbf{V}_{-}$and $\mathbf{V}_{+}$are then uniquely determined. It remains to check that the pair $\left(\mathbf{V}_{-}, \mathbf{V}_{+}\right)$satisfies the coupling constraints (108). The first coupling constraint $\mathbf{V}_{-} \in \mathcal{O}_{L}\left(\mathbf{V}_{+}\right)$holds if and only if the 1-wave of $\mathbf{Z}_{L}\left(. ; \mathbf{V}_{-}, \mathbf{V}_{+}\right)$has a nonnegative speed, i.e., by Proposition 5, if and only if

$$
u_{-} \geq c_{L}\left(p_{-}, s_{-}\right)
$$

and

Since by Lemma 4

$$
u_{L, s}^{1}\left(\mathbf{V}_{-}\right) \leq u_{+}+\Phi_{L, V_{+}}\left(p_{L, s}^{1}\left(\mathbf{V}_{-}\right)\right)
$$

$$
u_{-} \geq c_{L}\left(p_{-}, s_{-}\right)= \begin{cases}u_{G}-c_{L, G} \geq 0, & \text { if } \mathbf{V}_{-}=\mathbf{V}_{G}, \\ 0, & \text { if } \mathbf{V}_{-} \text {is the sonic state of the 1-wave }\end{cases}
$$

we have only to check the condition (110).

Similarly, we obtain that the second coupling constraint $\mathbf{V}_{+} \in \mathcal{O}_{R}\left(\mathbf{V}_{-}\right)$holds if and only if we have the condition

$$
u_{R, s}^{3}\left(\mathbf{V}_{+}\right) \geq u_{-}-\Phi_{R, V_{-}}\left(p_{R, s}^{3}\left(\mathbf{V}_{+}\right)\right)
$$

More precisely, the corresponding possible solutions are of the following forms.

6.1.a. When $\lambda_{L}^{1}\left(\mathbf{V}_{G}\right) \geq 0, \lambda_{R}^{3}\left(\mathbf{V}_{D}\right) \leq 0$, the solution is stationary: the states $\mathbf{V}_{G}$ and $\mathbf{V}_{D}$ are separated by a stationary discontinuity (cf. Section 5 ). 
6.1.b. Otherwise, the solution consists of one composite wave which separates the states $\mathbf{V}_{G}$ and $\mathbf{V}_{D}$ : this wave may have three admissible forms (see Fig. 11):

(i) for $\lambda_{L}^{1}\left(\mathbf{V}_{G}\right)<0, \lambda_{R}^{3}\left(\mathbf{V}_{D}\right) \leq 0$, the wave is composed of a 1-L-rarefaction followed by a stationary discontinuity in which case $\mathbf{V}_{-}$is the sonic state of the 1 - $L$-rarefaction and $\mathbf{V}_{+}=\mathbf{V}_{D}$;

(ii) for $\lambda_{L}^{1}\left(\mathbf{V}_{G}\right) \geq 0, \lambda_{R}^{3}\left(\mathbf{V}_{D}\right)>0$, the wave is composed of a stationary discontinuity followed by a 3 - $R$-rarefaction: here $\mathbf{V}_{-}=\mathbf{V}_{G}$ and $\mathbf{V}_{+}$is the sonic state of the $3-R$ rarefaction;

(iii) for $\lambda_{L}^{1}\left(\mathbf{V}_{G}\right)<0, \lambda_{R}^{3}\left(\mathbf{V}_{D}\right)>0$, the wave is composed of a 1-L-rarefaction followed by a stationary discontinuity and a 3 -rarefaction so that $\mathbf{V}_{-}$(resp. $\mathbf{V}_{+}$) is the sonic state of the 1 - $L$-rarefaction (resp. the 3 - $R$-rarefaction).

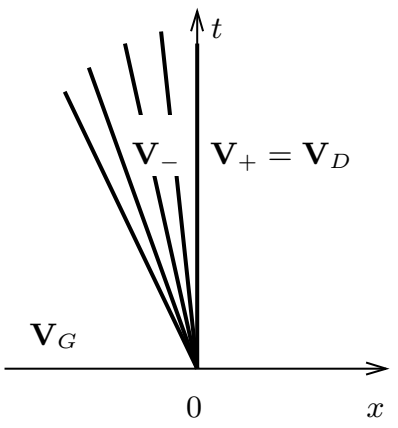

(i)

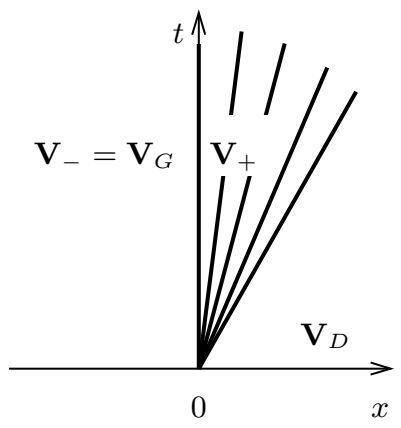

(ii)

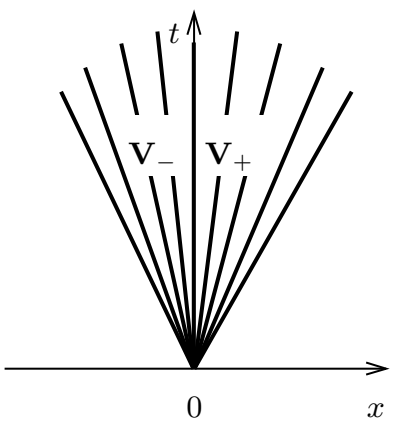

(iii)

FIG. 11. Case 6.1.b.

6.2. $\boldsymbol{Z}_{L}\left(. ; \boldsymbol{V}_{-}, \boldsymbol{V}_{+}\right)$has a 1 -wave and $\boldsymbol{Z}_{R}\left(. ; \boldsymbol{V}_{-}, \boldsymbol{V}_{+}\right)$has no 3-wave. Then we have

$$
\mathbf{V}_{-}= \begin{cases}\mathbf{V}_{G}, & \text { if } \lambda_{L}^{1}\left(\mathbf{V}_{G}\right) \geq 0 \\ \text { the sonic state of } \mathcal{C}_{L, r}^{1}\left(\mathbf{V}_{G}\right), & \text { if } \lambda_{L}^{1}\left(\mathbf{V}_{G}\right)<0\end{cases}
$$

and

$$
\mathbf{V}_{+}=\mathbf{Z}_{R}\left(0_{+} ; \mathbf{V}_{-}, \mathbf{V}_{D}\right)
$$

so that the pair $\left(\mathbf{V}_{-}, \mathbf{V}_{+}\right)$is again uniquely determined. Since the latter condition implies $\mathbf{V}_{+} \in \mathcal{O}_{R}\left(\mathbf{V}_{-}\right)$, we obtain here that the coupling constraints (108) resume to $\mathbf{V}_{-} \in \mathcal{O}_{L}\left(\mathbf{V}_{+}\right)$or equivalently to the condition (110). On the other hand, we observe that $\mathbf{Z}_{R}\left(0_{+} ; \mathbf{V}_{-}, \mathbf{V}_{D}\right)$ can be arbitrary.

We find solutions of the following forms.

6.2.a. For $\lambda_{L}^{1}\left(\mathbf{V}_{G}\right) \geq 0$, the states $\mathbf{V}_{G}$ and $\mathbf{V}_{D}$ are separated by a stationary discontinuity and at most three $R$-waves, namely the waves of $\mathbf{Z}_{R}\left(. ; \mathbf{V}_{G}, \mathbf{V}_{D}\right)$ whose speeds are nonnegative. Note that the stationary discontinuity may form a composite wave with either a stationary discontinuity wave of $\mathbf{Z}_{R}\left(. ; \mathbf{V}_{G}, \mathbf{V}_{D}\right)$ or a rarefaction wave of $\mathbf{Z}_{R}\left(. ; \mathbf{V}_{G}, \mathbf{V}_{D}\right)$ which has a sonic state (see Fig. 12). 


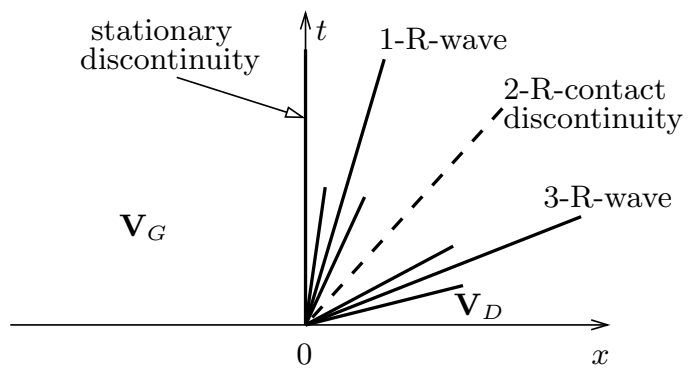

FIG. 12. Case 6.2.a.

6.2.b. For $\lambda_{L}^{1}\left(\mathbf{V}_{G}\right)<0$, the states $\mathbf{V}_{G}$ and $\mathbf{V}_{D}$ are separated by a composite wave formed by a 1 - $L$-rarefaction and a stationary discontinuity followed by at most three $R$-waves (see Fig. 13).

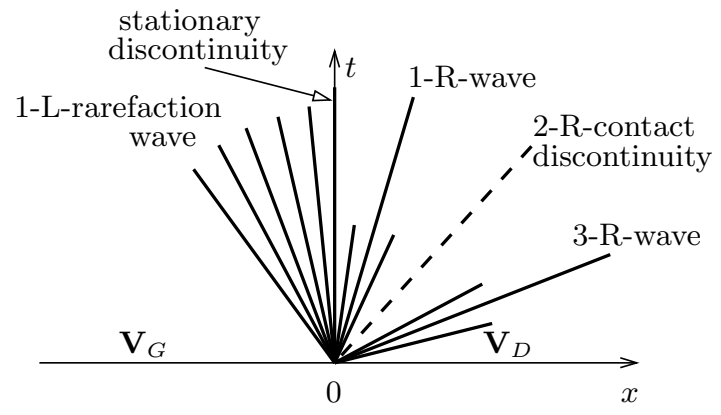

FIG. 13. Case 6.2.b.

Hence, in this case 6.2, the solution consists of at most five states (including $\mathbf{V}_{G}$ and $\mathbf{V}_{D}$ ) separated by four waves, the first one being a stationary discontinuity or a composite wave whose right edge is stationary.

6.3. $\boldsymbol{Z}_{L}\left(. ; \boldsymbol{V}_{-}, \boldsymbol{V}_{+}\right)$has no 1 -wave and $\boldsymbol{Z}_{R}\left(. ; \boldsymbol{V}_{-}, \boldsymbol{V}_{+}\right)$has a 3-wave. This is the symmetric situation of the previous case. Here we have

$$
\mathbf{V}_{+}= \begin{cases}\mathbf{V}_{D}, & \text { if } \lambda_{R}^{3}\left(\mathbf{V}_{D}\right) \leq 0 \\ \text { the sonic state of } \mathcal{C}_{R, r}^{3}\left(\mathbf{V}_{D}\right), & \text { if } \lambda_{R}^{3}\left(\mathbf{V}_{D}\right)>0\end{cases}
$$

and

$$
\mathbf{V}_{-}=\mathbf{Z}_{L}\left(0_{-} ; \mathbf{V}_{G}, \mathbf{V}_{+}\right)
$$

The coupling constraints (108) resume in this case to $\mathbf{V}_{+} \in \mathcal{O}_{R}\left(\mathbf{V}_{-}\right)$or equivalently to the condition (111). We obtain possible solutions of the forms

6.3.a. For $\lambda_{R}^{3}\left(\mathbf{V}_{D}\right) \leq 0$, the states $\mathbf{V}_{G}$ and $\mathbf{V}_{D}$ are separated by at most three $L$ waves, namely the waves of $\mathbf{Z}_{L}\left(. ; \mathbf{V}_{G}, \mathbf{V}_{D}\right)$ whose speeds are nonpositive, followed by a stationary discontinuity.

6.3.b. For $\lambda_{R}^{3}\left(\mathbf{V}_{D}\right)>0$, the states $\mathbf{V}_{G}$ and $\mathbf{V}_{D}$ are separated by at most three $L$-waves followed by a composite wave formed by a stationary discontinuity and a $3-R$ rarefaction wave. 
6.4. $\boldsymbol{Z}_{L}\left(. ; \boldsymbol{V}_{-}, \boldsymbol{V}_{+}\right)$has no 1-wave and $\boldsymbol{Z}_{R}\left(. ; \boldsymbol{V}_{-}, \boldsymbol{V}_{+}\right)$has no 3-wave. We find here

$$
\Upsilon_{-} \in \Gamma_{L}^{3}\left(\mathbf{V}_{+}\right), \Upsilon_{+} \in \Gamma_{R}^{1}\left(\mathbf{V}_{-}\right)
$$

Now, as in Section 5, we need to specify the variables $\mathbf{V}$.

If (99) holds, we use again the fact that the curves $\Gamma_{L}^{3}\left(\mathbf{V}_{+}\right)$and $\Gamma_{R}^{1}\left(\mathbf{V}_{-}\right)$intersect at one point at most to obtain

$$
u_{-}=u_{+}, p_{-}=p_{+} .
$$

Hence $\mathbf{Z}_{L}\left(. ; \mathbf{V}_{-}, \mathbf{V}_{+}\right)$consists in a 2 - $L$-contact discontinuity whose speed $u_{-}=u_{+}$is nonnegative while $\mathbf{Z}_{R}\left(. ; \mathbf{V}_{-}, \mathbf{V}_{+}\right)$consists in a $2-R$-contact discontinuity whose speed $u_{-}=u_{+}$is nonpositive. Then we have $u_{-}=u_{+}=0$ and in addition $\mathbf{V}_{-} \in \mathcal{C}_{L}^{1}\left(\mathbf{V}_{G}\right)$ and $\mathbf{V}_{+} \in \mathcal{C}_{R}^{3}\left(\mathbf{V}_{D}\right)$. On the other hand, since $\mathbf{V}_{-}=\mathbf{Z}_{L}\left(0_{-} ; \mathbf{V}_{-}, \mathbf{V}_{+}\right)$(resp. $\mathbf{V}_{+}=$ $\mathbf{Z}_{R}\left(0_{+} ; \mathbf{V}_{-}, \mathbf{V}_{+}\right)$), we get $\mathbf{V}_{-} \in \mathcal{O}_{L}\left(\mathbf{V}_{+}\right)$(resp. $\mathbf{V}_{+} \in \mathcal{O}_{R}\left(\mathbf{V}_{-}\right)$) and the coupling constraints are automatically satisfied.

We turn to a more general choice of the variables $\mathbf{V}$. Again we assume the hypothesis (103). Since $\mathbf{Z}_{L}\left(. ; \mathbf{V}_{-}, \mathbf{V}_{+}\right)$has no 1-wave and $\mathbf{Z}_{R}\left(. ; \mathbf{V}_{-}, \mathbf{V}_{+}\right)$has no 3-wave, we have respectively

$$
u_{-}=u_{+}+\Phi_{L, V_{+}}\left(p_{L}\left(\mathbf{V}_{-}\right)\right)=u_{+}+\Phi_{L, V_{+}}\left(\chi_{L}\left(\pi\left(\mathbf{V}_{-}\right)\right)\right)
$$

and

$$
u_{+}=u_{-}-\Phi_{R, V_{-}}\left(p_{R}\left(\mathbf{V}_{+}\right)\right)=u_{-}-\Phi_{R, V_{-}}\left(\chi_{R}\left(\pi\left(\mathbf{V}_{+}\right)\right)\right) .
$$

In other words, setting $\pi_{ \pm}=\pi\left(\mathbf{V}_{ \pm}\right)$, we get

$$
u_{-}=u_{+}+\tilde{\Phi}_{L, V_{+}}\left(\pi_{-}\right)
$$

and

$$
u_{+}=u_{-}-\tilde{\Phi}_{R, V_{-}}\left(\pi_{+}\right) .
$$

Hence, by projection onto the $(u, \pi)$-plane, we obtain here

$$
\left(u_{-}, \pi_{-}\right) \in \tilde{\Gamma}_{L}^{3}\left(\mathbf{V}_{+}\right),\left(u_{+}, \pi_{+}\right) \in \tilde{\Gamma}_{R}^{1}\left(\mathbf{V}_{-}\right),
$$

which yields

$$
u_{-}=u_{+}, \pi_{-}=\pi_{+},
$$

and therefore

$$
p_{\alpha}\left(\mathbf{V}_{-}\right)=\chi_{\alpha}\left(\pi_{-}\right)=\chi_{\alpha}\left(\pi_{+}\right)=p_{\alpha}\left(\mathbf{V}_{+}\right), \alpha=L, R .
$$

Again, we find that, for $\alpha=L, R, \mathbf{Z}_{\alpha}\left(. ; \mathbf{V}_{-}, \mathbf{V}_{+}\right)$consists of a 2 - $\alpha$-contact discontinuity whose velocity is $u_{-}=u_{+}$. Thus we have $u_{-}=u_{+}=0$ so that $\mathbf{V}_{-}=\mathbf{Z}_{L}\left(0_{-} ; \mathbf{V}_{-}, \mathbf{V}_{+}\right)$, $\mathbf{V}_{+}=\mathbf{Z}_{R}\left(0_{+} ; \mathbf{V}_{-}, \mathbf{V}_{+}\right)$and the coupling constraints (108) still hold.

If we suppose that either (99) or (103) holds, the solution of the coupled Riemann problem consists in general of the four states $\mathbf{V}_{G}, \mathbf{V}_{-}, \mathbf{V}_{+}$and $\mathbf{V}_{D}$ separated by a 1-Lwave, a stationary contact discontinuity and a 3 - $R$-wave (the $1-L$-wave and the 3 - $R$-wave may not exist). Such a solution, see Fig. 14, is uniquely determined. Indeed, assuming for instance the hypothesis (99), we obtain

$$
\begin{aligned}
& 0=u_{-}=u_{G}-\Phi_{L, G}\left(p_{-}\right), \\
& 0=u_{+}=u_{D}+\Phi_{R, D}\left(p_{+}\right)
\end{aligned}
$$


so that $p=p_{-}=p_{+}$satisfies

$$
\Phi_{L, G}(p)=u_{G}, \Phi_{R, D}(p)=u_{D},
$$

which uniquely defines the pressure $p$ (under the compatibility condition

$$
\left.\Phi_{L, G}^{-1}\left(u_{G}\right)=\Phi_{R, D}^{-1}\left(-u_{D}\right)\right) .
$$

On the other hand, we have

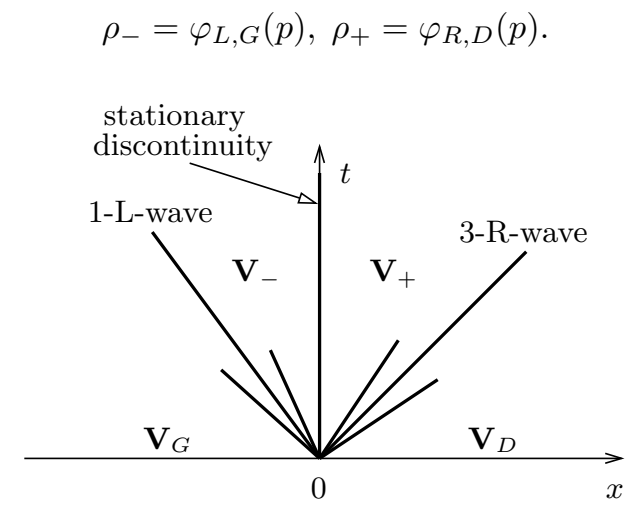

FIG. 14. The case with four states.

As a consequence of the above description of all possible solutions of the coupled Riemann problem which are $\mathbf{V}$-discontinuous at $x=0$, we note that there exists a fairly limited number of types of such solutions. Moreover, we can state

Theorem 5. The $\mathbf{V}$-coupled Riemann problem has at most one solution which is $\mathbf{V}$ discontinuous at the interface $x=0$.

7. An example of a solution of the V-coupled Riemann problem. It seems hopeless or at least fairly complicated to obtain explicit solutions to the $\mathbf{V}$-coupled Riemann problem for any pair $\left(\mathbf{U}_{G}, \mathbf{U}_{D}\right) \in \Omega^{2}$. Nevertheless, in order to illustrate the results of the previous sections, we want to construct explicitly these solutions in the special case

$$
u_{G}=u_{D}=u_{0} .
$$

This corresponds to the propagation of a matter wave through the interface. This case can also serve as a benchmark for testing the numerical methods of coupling.

Just for specificity we assume

$$
p_{G} \geq p_{D}
$$

the case $p_{G} \leq p_{D}$ being analyzed analogously, and we suppose that the hypothesis (99) holds, i.e., $\mathbf{V}=(\rho, u, p)^{T}$. We consider the curves $\Gamma_{L}^{1}\left(\mathbf{V}_{G}\right)$ and $\Gamma_{R}^{3}\left(\mathbf{V}_{D}\right)$ of the $(u, p)$ plane which intersect at point $\Upsilon^{*}=\left(u^{*}, p^{*}\right)$. If $p_{G}=p_{D}$, we note that $\Upsilon^{*}=\Upsilon_{G}=\Upsilon_{D}$ and the solution of the coupled-Riemann problem $\mathbf{Z}\left(. ; \mathbf{V}_{G}, \mathbf{V}_{D}\right)$ is a contact discontinuity:

$$
\mathbf{Z}\left(x / t ; \mathbf{V}_{G}, \mathbf{V}_{D}\right)= \begin{cases}\mathbf{V}_{G}, & \text { if } x / t<u_{0} \\ \mathbf{V}_{D}, & \text { if } x / t>u_{0}\end{cases}
$$


This is obvious if $u_{0} \neq 0$ and it follows from Theorem 4 (conditions (ii)) if $u_{0}=0$.

From now on, we assume $p_{G}>p_{D}$. We introduce a self-similar function $\mathbf{V}=\mathbf{V}(x / t)$ which can be viewed as a first candidate for the solution $\mathbf{Z}\left(x / t ; \mathbf{V}_{G}, \mathbf{V}_{D}\right)$ of the $\mathbf{V}$-coupled Riemann problem. Setting

$$
\rho_{G}^{*}=\varphi_{L, G}\left(p^{*}\right), \rho_{D}^{*}=\varphi_{R, D}\left(p^{*}\right), \mathbf{V}_{G}^{*}=\left(\rho_{G}^{*}, u^{*}, p^{*}\right)^{T}, \mathbf{V}_{D}^{*}=\left(\rho_{D}^{*}, u^{*}, p^{*}\right)^{T},
$$

this function consists of the constant states $\mathbf{V}_{G}, \mathbf{V}_{G}^{*}, \mathbf{V}_{D}^{*}$ and $\mathbf{V}_{D}$ separated by a 1-Lrarefaction wave whose extreme speeds are $\sigma_{1}<\sigma_{2}$, a contact discontinuity with speed $u^{*}$ and a 3 - $R$-shock wave with speed $\sigma_{3}$ (see Fig. 15).

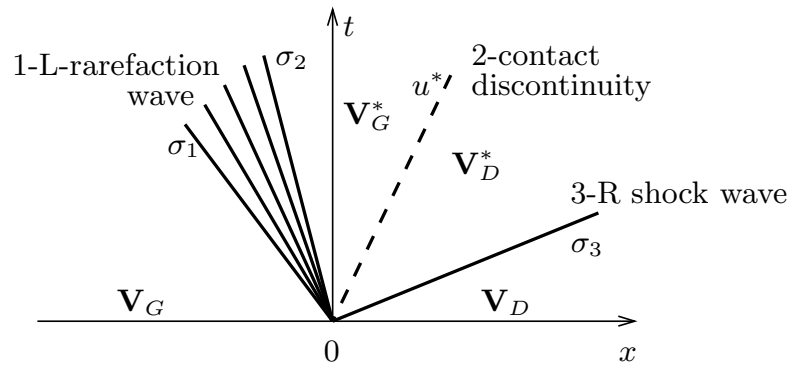

FIG. 15. The initial example.

Note that this function is indeed a solution of the $\mathbf{V}$-coupled Riemann problem when $\sigma_{2} \leq 0<\sigma_{3}$. For $\sigma_{2}>0$ or $\sigma_{3}<06$ this solution is no longer admissible. We are thus led to distinguish several cases depending on the signs of $\sigma_{1}, \sigma_{2}$ and $\sigma_{3}$.

7.1. The case $\sigma_{1} \geq 0$.

If $\sigma_{1}=u_{G}-c_{L, G}=u_{0}-c_{L, G} \geq 0$, we expect the solution of the coupled Riemann problem to coincide with $\mathbf{V}_{G}$ in the quadrant $(x>0, t>0)$. We thus consider the solution $\tilde{\mathbf{V}}=\mathbf{Z}_{R}\left(. ; \mathbf{V}_{G}, \mathbf{V}_{D}\right)$ of the $R$-Riemann problem. We denote by $\tilde{\Upsilon}^{*}=\left(\tilde{u}^{*}, \tilde{p}^{*}\right)$ the intersection point of the curves $\Gamma_{R}^{1}\left(\mathbf{V}_{G}\right)$ and $\Gamma_{R}^{3}\left(\mathbf{V}_{D}\right)$. Clearly we have

$$
u^{*}>u_{0}>0, \tilde{u}^{*}>u_{0}>0 .
$$

This function $\tilde{\mathbf{V}}$ again consists of the constant states $\mathbf{V}_{G}, \tilde{\mathbf{V}}_{G}^{*}, \tilde{\mathbf{V}}_{D}^{*}$ and $\mathbf{V}_{D}$ separated by a 1- $R$-rarefaction wave whose extreme speeds are $\tilde{\sigma}_{1}<\tilde{\sigma}_{2}$, a 2 - $R$-contact discontinuity with velocity $\tilde{u}^{*}$ and a 3 - $R$-shock wave with speed $\tilde{\sigma}_{3}$ (see Fig. 16).

7.1.1. The case $\sigma_{1} \geq 0, \tilde{\sigma}_{1} \geq 0$.

A solution of the coupled Riemann problem is that of the $R$-Riemann problem, i.e.,

$$
\mathbf{Z}\left(. ; \mathbf{V}_{G}, \mathbf{V}_{D}\right)=\mathbf{Z}_{R}\left(. ; \mathbf{V}_{G}, \mathbf{V}_{D}\right) .
$$

Indeed, this corresponds to the case $\mathbf{V}_{G} \in \mathcal{V}_{R}^{3}\left(\mathbf{V}_{D}\right)$.

7.1.2. The case $\sigma_{1} \geq 0, \tilde{\sigma}_{1}<0, \tilde{\sigma}_{2} \geq 0$.

We postulate here that a solution of the coupled Riemann problem is given by

$$
\mathbf{Z}\left(x / t ; \mathbf{V}_{G}, \mathbf{V}_{D}\right)= \begin{cases}\mathbf{V}_{G}, & \text { if } x / t<0, \\ \tilde{\mathbf{V}}(x / t)=\mathbf{Z}_{R}\left(x / t ; \mathbf{V}_{G}, \mathbf{V}_{D}\right), & \text { if } x / t>0 .\end{cases}
$$

\footnotetext{
${ }^{6}$ When $\sigma_{3}=0$, we need to check whether the pair $\left(\mathbf{V}_{D}^{*}, \mathbf{V}_{D}\right)$ satisfies the coupling constraints or not.
} 


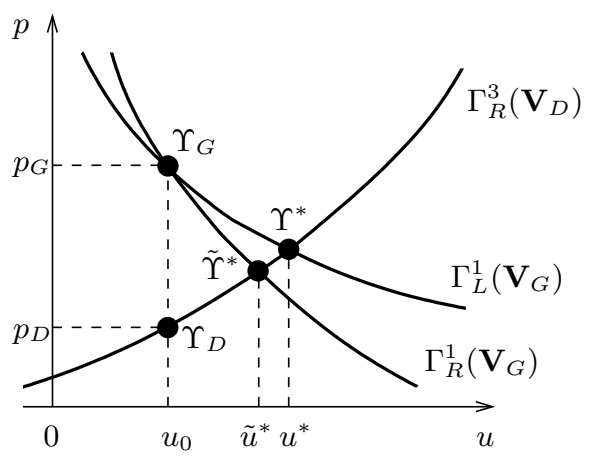

FiG. 16. The case 7.1: $\sigma_{1} \geq 0$.

It consists of the four states $\mathbf{V}_{G}, \tilde{\mathbf{V}}_{G}^{*}, \tilde{\mathbf{V}}_{D}^{*}$ and $\mathbf{V}_{D}$ separated by a composite wave formed of a stationary discontinuity and a 1-R-rarefaction, a 2 - $R$-contact discontinuity and a 3 - $R$-shock. This function is discontinuous at $x=0$ with

$$
\mathbf{Z}\left(0_{-}\right)=\mathbf{V}_{G}, \mathbf{Z}\left(0_{+}\right)=\tilde{\mathbf{V}}(0) .
$$

Note that $\tilde{\mathbf{V}}(0)$ is the sonic state of the 1 - $R$-rarefaction wave. This is in fact a solution of the form considered in Paragraph 6.2.a (see Fig. 17).
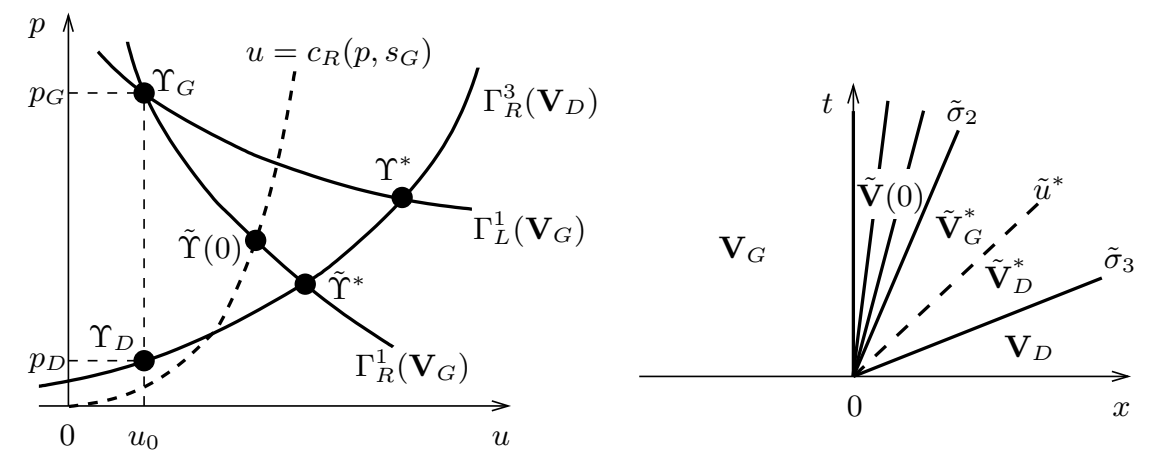

FIG. 17

It remains to check that $\mathbf{V}_{G}$ and $\tilde{\mathbf{V}}(0)$ satisfy the coupling constraints. On the one hand, we observe (cf. Fig. 17) that the solution of the $L$-Riemann problem $\mathbf{Z}_{L}\left(. ; \mathbf{V}_{G}\right.$, $\tilde{\mathbf{V}}(0)$ ) contains necessarily a 1-wave. On the other hand, $\tilde{\mathbf{V}}(0)$ is connected to $\mathbf{V}_{G}$ by a 1- $R$-wave so that $\mathbf{Z}_{R}\left(. ; \mathbf{V}_{G}, \tilde{\mathbf{V}}(0)\right)$ has no 3-wave. In addition, we have by construction $\tilde{\mathbf{V}}(0)=\mathbf{Z}_{R}\left(0 ; \mathbf{V}_{G}, \mathbf{V}_{D}\right)$. Hence, by the results of Paragraph 6.2 , we have only to verify the constraint $\mathbf{V}_{G} \in \mathcal{O}_{L}(\tilde{\mathbf{V}}(0))$. Since the conditions $\tilde{\sigma}_{1}<0 \leq \sigma_{1}$ mean

$$
u_{0}-c_{R, G}<0 \leq u_{0}-c_{L, G},
$$

we obtain (cf. again Fig. 17) that $\tilde{\Upsilon}(0)$ is located below $\Upsilon_{G}$ on the curve $\Gamma_{R}^{1}\left(\mathbf{V}_{G}\right)$ and therefore that the 1-wave of $\mathbf{Z}_{L}\left(. ; \mathbf{V}_{G}, \tilde{\mathbf{V}}(0)\right)$ is a rarefaction whose speed is nonnegative, 
which yields

$$
\mathbf{Z}_{L}\left(0 ; \mathbf{V}_{G}, \tilde{\mathbf{V}}(0)\right)=\mathbf{V}_{G}
$$

and the desired property follows.

7.1.3. The case $\sigma_{1} \geq 0, \tilde{\sigma}_{2}<0$.

Again we postulate that the solution is given by (114); i.e., it consists here of the four states $\mathbf{V}_{G}, \tilde{\mathbf{V}}_{G}^{*}, \tilde{\mathbf{V}}_{D}^{*}$ and $\mathbf{V}_{D}$ separated by a stationary discontinuity and a 1$R$-rarefaction, a $2-R$-contact discontinuity and a 3 - $R$-shock. This function is still discontinuous at $x=0$ :

$$
\mathbf{Z}\left(0_{-}\right)=\mathbf{V}_{G}, \mathbf{Z}\left(0_{+}\right)=\tilde{\mathbf{V}}_{G}^{*}
$$
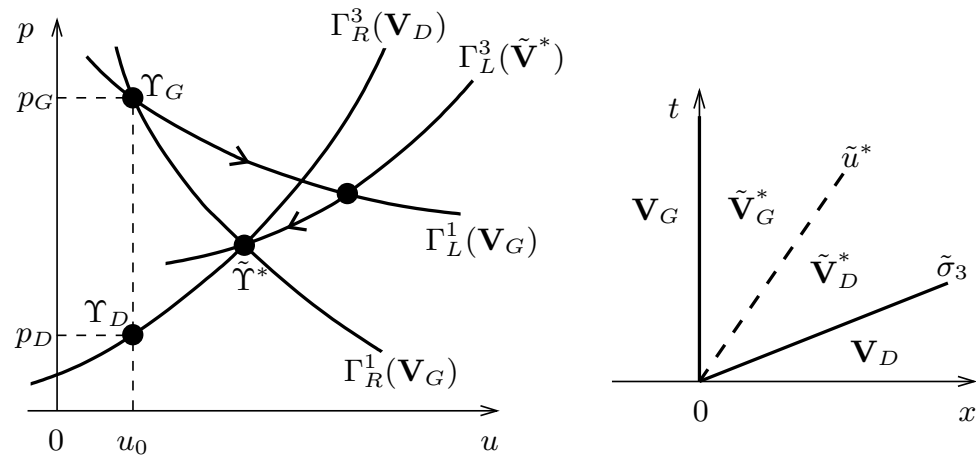

FIG. 18

In order to check that this discontinuity satisfies the coupling constraints, we argue exactly as in Paragraph 7.1.2. We observe (cf. Fig. 18) that $\mathbf{Z}_{L}\left(. ; \mathbf{V}_{G}, \tilde{\mathbf{V}}_{G}^{*}\right)$ contains necessarily a 1-wave and $\tilde{\mathbf{V}}_{G}^{*}$ is connected to $\mathbf{V}_{G}$ by a 1- $R$-wave. Since $\tilde{\mathbf{V}}_{G}^{*}=\mathbf{Z}_{R}\left(0_{+} ; \mathbf{V}_{G}, \mathbf{V}_{D}\right)$, we have only to check $\mathbf{V}_{G} \in \mathcal{O}_{L}\left(\tilde{\mathbf{V}}_{G}^{*}\right)$. This follows at once from

$$
\mathbf{Z}_{L}\left(0_{-} ; \mathbf{V}_{G}, \tilde{\mathbf{V}}_{G}^{*}\right)=\mathbf{V}_{G} \text {. }
$$

7.2. The case $\sigma_{1}<0, \sigma_{2} \geq 0$.

From now on we suppose $\sigma_{1}=u_{0}-c_{L, G}<0$ and in this subsection we consider the case

$$
\sigma_{2}=u^{*}-c_{L}\left(p^{*}, s_{G}\right) \geq 0 .
$$

Then we expect the solution $\mathbf{Z}\left(. ; \mathbf{V}_{G}, \mathbf{V}_{D}\right)$ of the coupled Riemann problem to coincide with the function $\mathbf{V}$ in the quadrant $(x<0, t>0)$. Note that $\mathbf{V}(0)$ is the sonic state of a 1 - $L$-rarefaction, i.e.,

$$
u(0)=c_{L}\left(p^{*}, s_{G}\right)
$$

Thus we introduce the function $\check{\mathbf{V}}=\mathbf{Z}_{R}\left(. ; \mathbf{V}(0), \mathbf{V}_{D}\right)$, i.e., the solution of the Riemann problem associated with the pair $\left(\mathbf{V}(0), \mathbf{V}_{D}\right)$. We denote by $\check{\Upsilon}^{*}=\left(\check{u}^{*}, \check{p}^{*}\right)$ the intersection point of the curves $\Gamma_{R}^{1}(\mathbf{V}(0))$ and $\Gamma_{R}^{3}\left(\mathbf{V}_{D}\right)$. This function $\mathbf{V}$ consists the of constant states $\mathbf{V}(0), \check{\mathbf{V}}(0)^{*}, \check{\mathbf{V}}_{D}^{*}$ and $\mathbf{V}_{D}$ separated by a $1-R$-rarefaction wave with end speeds $\check{\sigma}_{1}<\check{\sigma}_{2}$, a 2 - $R$-contact discontinuity with speed $\check{u}^{*}$ and a 3 - $R$-shock wave with speed $\check{\sigma}_{3}$. Then we observe that

$$
\check{\sigma}_{1}=u(0)-c_{R}\left(p(0), s_{G}\right)=c_{L}\left(p(0), s_{G}\right)-c_{R}\left(p(0), s_{G}\right) .
$$


Hence the sign of $\check{\sigma}_{1}$ depends on the sound speeds $c_{L}$ and $c_{R}$. Again we distinguish several subcases according to the signs of $\check{\sigma}_{1}$ and $\check{\sigma}_{2}$.

7.2.1. The case $\sigma_{1}<0, \sigma_{2} \geq 0, \check{\sigma}_{1}>0$.

This subcase corresponds to

$$
c_{L}\left(p(0), s_{G}\right)>c_{R}\left(p(0), s_{G}\right) .
$$

A possible solution of the coupled Riemann problem then consists of the five constant states $\mathbf{V}_{G}, \mathbf{V}(0), \check{\mathbf{V}}(0)^{*}, \check{\mathbf{V}}_{D}^{*}$ and $\mathbf{V}_{D}$ separated by a 1 - $L$-rarefaction wave whose end speeds are $\sigma_{1}$ and 0 , a 1-R-rarefaction wave whose end velocities are $\check{\sigma}_{1}$ and $\check{\sigma}_{2}$, a 2 $R$-contact discontinuity with speed $\check{u}^{*}$ and a 3 - $R$-shock wave with speed $\check{\sigma}_{3}$. Such a solution is $\mathbf{V}$-continuous at $x=0$ and corresponds to the case (iii) of Section 4 . Indeed, we have clearly $\mathbf{V}(0) \in \hat{\mathcal{C}}_{L,-}^{1}\left(\mathbf{V}_{G}\right)$ while the condition $\check{\sigma}_{1}=u_{0}-c_{R}\left(p(0), s_{G}\right)>0$ implies $\mathbf{V}(0) \in \hat{\mathcal{V}}_{R,+}^{3}\left(\mathbf{V}_{D}\right)$ so that $\mathbf{V}(0) \in \hat{\mathcal{C}}_{L,-}^{1}\left(\mathbf{V}_{G}\right) \cap \hat{\mathcal{V}}_{R,+}^{3}\left(\mathbf{V}_{D}\right)$, as expected.

However, we know from the general study of Section 4 that in such a case there exists a one-parameter family of solutions. In fact, this family can be constructed in the following way. Choose any state $\mathbf{V}(\xi), \sigma_{1}<\xi<0$, of the 1 - $L$-rarefaction wave such that in addition

$$
u(\xi)-c_{R}\left(p(\xi), s_{G}\right)>0 .
$$

This latter condition holds at least for $|\xi|$ small enough. Then we have $\mathbf{V}(\xi) \in \hat{\mathcal{C}}_{L,-}^{1}\left(\mathbf{V}_{G}\right) \cap$ $\hat{\mathcal{V}}_{R,+}^{3}\left(\mathbf{V}_{D}\right)$ and the function

$$
\mathbf{Z}\left(x / t ; \mathbf{V}_{G}, \mathbf{V}_{D}\right)= \begin{cases}\mathbf{V}(x / t), & \text { if } x / t \leq \xi, \\ \mathbf{V}(\xi), & \text { if } \xi \leq x / t \leq 0, \\ \mathbf{Z}_{R}\left(x / t ; \mathbf{V}(\xi), \mathbf{V}_{D}\right), & \text { if } x / t \geq 0\end{cases}
$$

is clearly a solution of the coupled Riemann problem. Moreover, for

$$
u_{0}-c_{R, G}>0,
$$

we can even take $\xi=\sigma_{1}$ so that $\mathbf{Z}\left(x / t ; \mathbf{V}_{G}, \mathbf{V}_{D}\right)=\mathbf{Z}_{R}\left(x / t ; \mathbf{V}_{G}, \mathbf{V}_{D}\right)$. This corresponds to the case $\mathbf{V}_{G} \in \hat{\mathcal{V}}_{R,+}^{3}\left(\mathbf{V}_{D}\right)$ (cf. (82)).

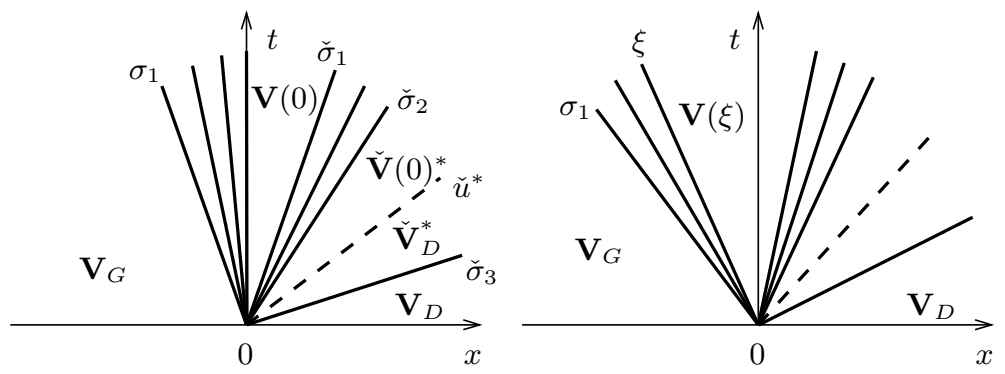

FIG. 19

7.2.2. The case $\sigma_{1}<0, \sigma_{2} \geq 0, \check{\sigma}_{1}<0, \check{\sigma}_{2} \geq 0$.

Let us now suppose $\check{\sigma}_{1}<0 \leq \check{\sigma}_{2}$, i.e.,

$$
c_{L}\left(p(0), s_{G}\right)<c_{R}\left(p(0), s_{G}\right), \check{u}^{*} \geq c_{R}\left(\check{p}(0)^{*}, s_{G}\right) .
$$


We postulate here that a solution $\mathbf{Z}\left(. ; \mathbf{V}_{G}, \mathbf{V}_{D}\right)$ of the coupled Riemann problem consists of the four states $\mathbf{V}_{G}, \check{\mathbf{V}}(0)^{*}, \check{\mathbf{V}}_{D}^{*}$ and $\mathbf{V}_{D}$ separated by a composite wave formed of a 1- $L$-rarefaction wave with end speeds $\sigma_{1}$ and 0 , a stationary discontinuity and a 1-Rrarefaction wave with end speeds 0 and $\check{\sigma}_{2}$, by a 2 - $R$-contact discontinuity with speed $\check{u}^{*}$ and a $3-R$-shock wave with speed $\check{\sigma}_{3}$ (see Fig. 20). This function is discontinuous at $x=0$ :

$$
\mathbf{Z}\left(0_{-}\right)=\mathbf{V}(0), \mathbf{Z}\left(0_{+}\right)=\check{\mathbf{V}}(0) .
$$

Again we have to check the coupling constraints. On the one hand, $\check{\mathbf{V}}(0)$ is connected to $\mathbf{V}(0)$ by a 1- $R$-rarefaction wave (cf. Fig. 20) and we have by construction

$$
\check{\mathbf{V}}(0)=\mathbf{Z}_{R}\left(0_{+} ; \mathbf{V}(0), \mathbf{V}_{D}\right)
$$

which proves that $\check{\mathbf{V}}(0) \in \mathcal{O}_{R}(\mathbf{V}(0))$. On the other hand, in order to check that $\mathbf{V}(0) \in$ $\mathcal{O}_{L}(\check{\mathbf{V}}(0))$ or equivalently by Lemma 3 that

$$
\mathbf{V}(0)=\mathbf{Z}_{L}\left(0_{-} ; \mathbf{V}(0), \check{\mathbf{V}}(0)\right),
$$

we remark that $\mathbf{Z}_{L}(. ; \mathbf{V}(0), \check{\mathbf{V}}(0))$ possesses a 1-wave which is a rarefaction whose speed is nonnegative (cf. Fig. 20). Hence the discontinuity is admissible. Again this is the situation encountered in the case 6.2 of Section 6 : $\mathbf{Z}_{L}(. ; \mathbf{V}(0), \check{\mathbf{V}}(0))$ has a 1-wave while $\mathbf{Z}_{R}(. ; \mathbf{V}(0), \check{\mathbf{V}}(0))$ has no 3 -wave.
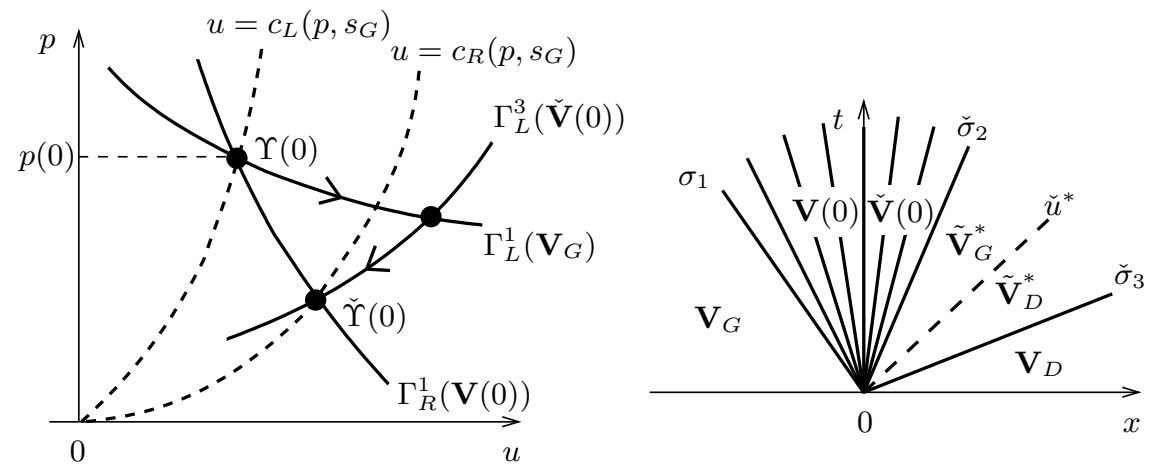

FIG. 20

7.2.3. The case $\sigma_{1}<0, \sigma_{2} \geq 0, \check{\sigma}_{2}<0$.

Let us now assume $\check{\sigma}_{2}<0$, i.e.,

$$
\check{u}^{*}<c_{R}\left(\check{p}^{*}, s_{G}\right) .
$$

We first notice that $\check{u}^{*}>0$. Indeed, since $\sigma_{2} \geq 0$, the point $\Upsilon^{*}$ is located below $\Upsilon(0)$ on the curve $\Gamma_{L}^{1}\left(\mathbf{V}_{G}\right)$, which means that $\Upsilon(0)$ is located above $\Gamma_{R}^{3}\left(\mathbf{V}_{D}\right)$. Hence the point $\check{\Upsilon}^{*}$ is located below $\Upsilon(0)$ on the curve $\Gamma_{R}^{1}(\mathbf{V}(0))$ so that (cf. Fig. 21)

$$
\check{u}^{*}>u(0)=c_{L}\left(p(0), s_{G}\right)>0 .
$$

We thus postulate that a solution $\mathbf{Z}\left(. ; \mathbf{V}_{G}, \mathbf{V}_{D}\right)$ of the coupled Riemann problem consists of the four states $\mathbf{V}_{G}, \check{\mathbf{V}}(0)^{*}, \check{\mathbf{V}}_{D}^{*}$ and $\mathbf{V}_{D}$ separated by a composite wave formed of a 1- $L$-rarefaction wave with end speeds $\sigma_{1}$ and 0 and of a stationary discontinuity, by a 
2-R-contact discontinuity with speed $\check{u}^{*}$ and a 3 - $R$-shock wave with speed $\check{\sigma}_{3}$. Again the proposed solution is discontinuous at $x=0$ with

$$
\mathbf{Z}\left(0_{-}\right)=\mathbf{V}(0), \mathbf{Z}\left(0_{+}\right)=\check{\mathbf{V}}(0)^{*}
$$

and we have to check the coupling constraints. This is done along the same lines as in the previous case. The state $\check{\mathbf{V}}(0)^{*}$ is connected to $\mathbf{V}(0)$ by a 1 - $R$-rarefaction wave and we have by construction

$$
\check{\mathbf{V}}(0)^{*}=\mathbf{Z}_{R}\left(0_{+} ; \mathbf{V}(0), \mathbf{V}_{D}\right)
$$

so that $\check{\mathbf{V}}(0)^{*} \in \mathcal{O}_{R}(\mathbf{V}(0))$. In addition, $\mathbf{Z}_{L}\left(. ; \mathbf{V}(0), \check{\mathbf{V}}(0)^{*}\right)$ has a 1-rarefaction wave whose speed is nonnegative, which yields

$$
\mathbf{V}(0)=\mathbf{Z}_{L}\left(0 ; \mathbf{V}(0), \check{\mathbf{V}}(0)^{*}\right)
$$

or equivalently $\mathbf{V}(0) \in \mathcal{O}_{L}\left(\check{\mathbf{V}}(0)^{*}\right)$. The discontinuity is therefore admissible.
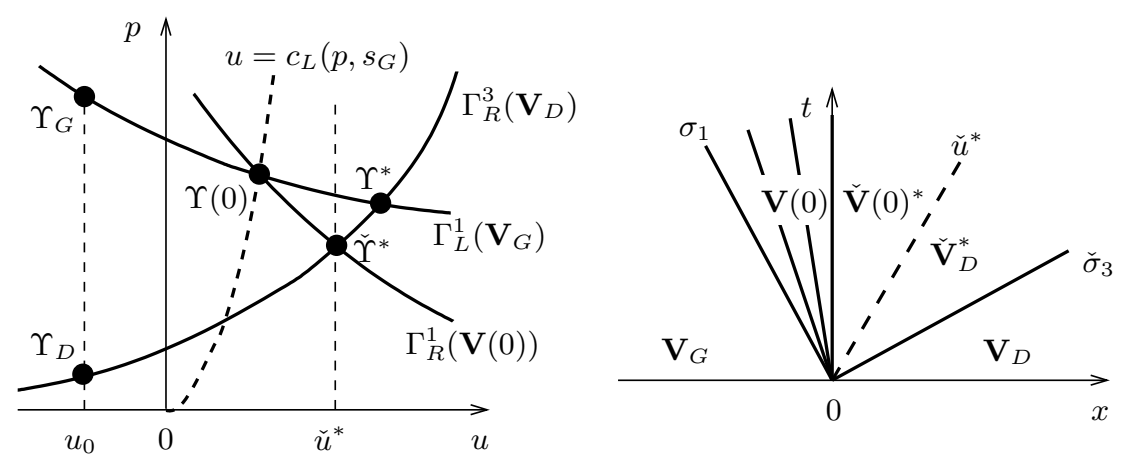

FIG. 21

7.3. The case $\sigma_{2}<0, \sigma_{3}>0$.

A solution of the $\mathbf{V}$-coupled Riemann problem is clearly the function $\mathbf{V}$ introduced at the beginning of this section.

7.4. The case $\sigma_{3} \leq 0$.

Let us finally assume $\sigma_{3} \leq 0$. Then it seems fairly natural to look for a solution of the coupled Riemann problem which coincides with $\mathbf{V}_{D}$ in the quadrant $(x>0, t>0)$. We thus introduce the solution $\overline{\mathbf{V}}=\mathbf{Z}_{L}\left(. ; \mathbf{V}_{G}, \mathbf{V}_{D}\right)$ of the $L$-Riemann problem. We denote by $\bar{\Upsilon}^{*}=\left(\bar{u}^{*}, \bar{p}^{*}\right)$ the intersection point of the curves $\Gamma_{L}^{1}\left(\mathbf{V}_{G}\right)$ and $\Gamma_{L}^{3}\left(\mathbf{V}_{D}\right)$. Again the function $\overline{\mathbf{V}}$ consists of four constant states $\mathbf{V}_{G}, \overline{\mathbf{V}}_{G}^{*}, \overline{\mathbf{V}}_{D}^{*}$ and $\mathbf{V}_{D}$ separated by a 1-Lrarefaction wave with end speeds $\bar{\sigma}_{1}$ and $\bar{\sigma}_{2}$, by a 2 - $L$-contact discontinuity with speed $\bar{u}^{*}$ and a 3 - $L$-shock wave with speed $\bar{\sigma}_{3}$ (see Fig. 22). Note that

$$
\bar{\sigma}_{1}=\sigma_{1}=u_{G}-c_{L, G}<0 .
$$

Again we have to distinguish several cases according to the sign of $\bar{\sigma}_{3}$.

7.4.1. The case $\sigma_{3} \leq 0, \bar{\sigma}_{3} \leq 0$.

When $\bar{\sigma}_{3}$ is nonpositive, an admissible solution of the $\mathbf{V}$-coupled Riemann problem is obviously the solution of the $L$-Riemann problem $\mathbf{Z}_{L}\left(. ; \mathbf{V}_{G}, \mathbf{V}_{D}\right)$. 

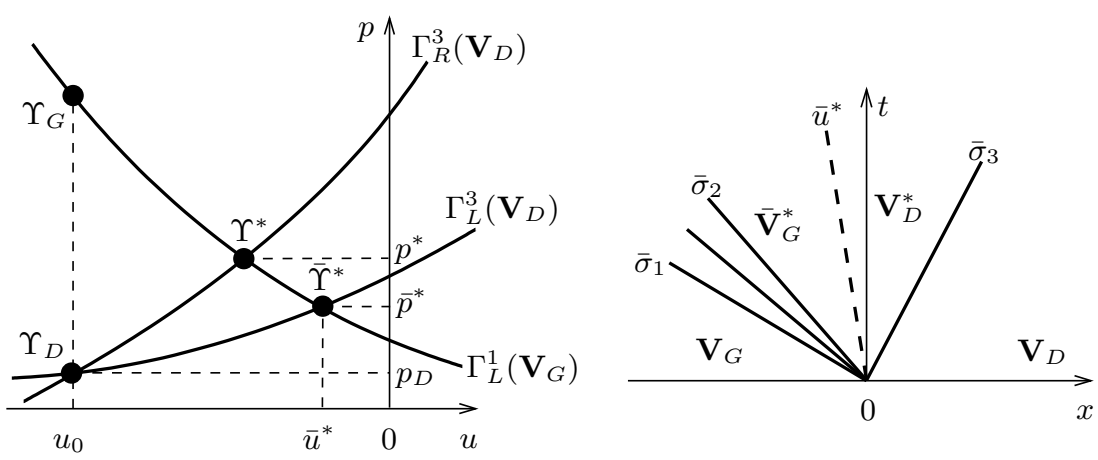

FIG. 22

7.4.2. The case $\sigma_{3} \leq 0, \bar{\sigma}_{3}>0$.

This is by far the most complex case. Let us first derive some consequences of the double inequality $\sigma_{3} \leq 0<\bar{\sigma}_{3}$. We begin by characterizing the states $\mathbf{V}_{D}^{*}$ and $\overline{\mathbf{V}}_{D}^{*}$. Recall that the intersection point $\Upsilon^{*}=\left(u^{*}, p^{*}\right)$ of the curves $\Gamma_{L}^{1}\left(\mathbf{V}_{G}\right)$ and $\Gamma_{R}^{3}\left(\mathbf{V}_{D}\right)$ is given by

$$
u^{*}=u_{0}-\Phi_{L, G}\left(p^{*}\right)=u_{0}+\Phi_{R, D}\left(p^{*}\right) .
$$

Since $\mathbf{V}_{D}$ and $\mathbf{V}_{D}^{*}$ are connected by a shock, we have $p^{*} \geq p_{D}$, which yields

$$
u^{*}=u_{0}+\sqrt{\left(p^{*}-p_{D}\right)\left(\frac{1}{\rho_{D}}-h_{R, D}\left(p^{*}\right)\right)}
$$

and

$$
\rho_{D}^{*}=\frac{1}{h_{R, D}\left(p^{*}\right)}>\rho_{D} .
$$

In a similar manner, the intersection point $\bar{\Upsilon}^{*}=\left(\bar{u}^{*}, \bar{p}^{*}\right)$ of the curves $\Gamma_{L}^{1}\left(\mathbf{V}_{G}\right)$ and $\Gamma_{L}^{3}\left(\mathbf{V}_{D}\right)$ is given by

$$
u^{*}=u_{0}-\Phi_{L, G}\left(p^{*}\right)=u_{0}+\Phi_{L, D}\left(p^{*}\right) .
$$

We have $\bar{p}^{*}>p_{D}$ and

$$
\begin{gathered}
\bar{u}^{*}=u_{0}+\sqrt{\left(\bar{p}^{*}-p_{D}\right)\left(\frac{1}{\rho_{D}}-h_{L, D}\left(\bar{p}^{*}\right)\right)}, \\
\bar{\rho}_{D}^{*}=\frac{1}{h_{L, D}\left(\bar{p}^{*}\right)}>\rho_{D} .
\end{gathered}
$$

Next, since

$$
\sigma_{3}=\frac{\rho_{D} u_{D}-\rho_{D}^{*} u_{D}^{*}}{\rho_{D}-\rho_{D}^{*}}=\frac{\rho_{D} u_{0}-\rho_{D}^{*} u^{*}}{\rho_{D}-\rho_{D}^{*}}
$$

the inequality $\sigma_{3} \leq 0$ reads

$$
u^{*} \leq \frac{\rho_{D}}{\rho_{D}^{*}} u_{0}
$$

and by (115), (116),

$$
u_{0}+\sqrt{\left(p^{*}-p_{D}\right)\left(\frac{1}{\rho_{D}}-h_{R, D}\left(p^{*}\right)\right)} \leq \rho_{D} h_{R, D}\left(p^{*}\right) u_{0}
$$


or equivalently

$$
-\left(1-\rho_{D} h_{R, D}\left(p^{*}\right)\right) u_{0} \geq \sqrt{\frac{1}{\rho_{D}}\left(p^{*}-p_{D}\right)\left(1-\rho_{D} h_{R, D}\left(p^{*}\right)\right)} .
$$

As $u_{0}$ is $<0$, we obtain

$$
\sigma_{3} \leq 0 \Leftrightarrow \frac{p^{*}-p_{D}}{1-\rho_{D} h_{R, D}\left(p^{*}\right)} \leq \rho_{D} u_{0}^{2} .
$$

On the other hand, since

$$
\bar{\sigma}_{3}=\frac{\rho_{D} u_{D}-\bar{\rho}_{D}^{*} \bar{u}_{D}^{*}}{\rho_{D}-\bar{\rho}_{D}^{*}}=\frac{\rho_{D} u_{0}-\bar{\rho}_{D}^{*} \bar{u}^{*}}{\rho_{D}-\bar{\rho}_{D}^{*}}
$$

the inequality $\bar{\sigma}_{3}>0$ gives

$$
\bar{u}^{*}>\frac{\rho_{D}}{\bar{\rho}_{D}^{*}} u_{0}
$$

and by (117), (118),

$$
u_{0}+\sqrt{\left(\bar{p}^{*}-p_{D}\right)\left(\frac{1}{\rho_{D}}-h_{L, D}\left(\bar{p}^{*}\right)\right)}>\rho_{D} h_{L, D}\left(\bar{p}^{*}\right) u_{0}
$$

so that

$$
\bar{\sigma}_{3}>0 \Leftrightarrow \frac{\bar{p}^{*}-p_{D}}{1-\rho_{D} h_{L, D}\left(\bar{p}^{*}\right)}>\rho_{D} u_{0}^{2} .
$$

Hence the double inequality $\sigma_{3} \leq 0<\bar{\sigma}_{3}$ implies

$$
\frac{p^{*}-p_{D}}{1-\rho_{D} h_{R, D}\left(p^{*}\right)}<\frac{\bar{p}^{*}-p_{D}}{1-\rho_{D} h_{L, D}\left(\bar{p}^{*}\right)} .
$$

Now, for the sake of simplicity, it is convenient to suppose that the equations of state $p=p_{\alpha}(\rho, \varepsilon), \alpha=L, R$, satisfy the following hypothesis:

H.3. For any state $\boldsymbol{V}_{0}$, we have either

$$
\begin{cases}\Phi_{L, 0}(p)>\Phi_{R, 0}(p), & \text { if } p>p_{0}, \\ \Phi_{L, 0}(p)<\Phi_{R, 0}(p), & \text { if } p<p_{0}\end{cases}
$$

or

$$
\begin{cases}\Phi_{L, 0}(p)<\Phi_{R, 0}(p), & \text { if } p>p_{0} \\ \Phi_{L, 0}(p)>\Phi_{R, 0}(p), & \text { if } p<p_{0}\end{cases}
$$

In other words, for any $i \in\{1,3\}$, the curves $\Gamma_{L}^{i}\left(\mathbf{V}_{0}\right)$ and $\Gamma_{R}^{i}\left(\mathbf{V}_{0}\right)$ intersect only at the point $\Upsilon_{0}=\left(u_{0}, p_{0}\right)$. In fact, it is an easy matter to check that this hypothesis is indeed satisfied for the polytropic equations of state (100): (120) holds for $\gamma_{R}>\gamma_{L}$ while (121) holds for $\gamma_{L}>\gamma_{R}$.

Using hypothesis H.3, we now check that the inequality (119) implies that the curve $\Gamma_{L}^{3}\left(\mathbf{V}_{D}\right)$ is located above $\Gamma_{R}^{3}\left(\mathbf{V}_{D}\right)$ for $p>p_{D}$ (or $\left.u>u_{0}\right)$. Let us assume on the contrary that $\Gamma_{R}^{3}\left(\mathbf{V}_{D}\right)$ is located above $\Gamma_{L}^{3}\left(\mathbf{V}_{D}\right)$ for $p>p_{D}$, i.e.,

$$
\Phi_{L, D}(p)=\sqrt{\left(p-p_{D}\right)\left(\frac{1}{\rho_{D}}-h_{L, D}(p)\right)}>\Phi_{R, D}(p)=\sqrt{\left(p-p_{D}\right)\left(\frac{1}{\rho_{D}}-h_{R, D}(p)\right)}
$$

or equivalently,

$$
h_{R, D}(p)>h_{L, D}(p), p>p_{D} .
$$


Then we have $\bar{p}^{*}<p^{*}$ (cf. Fig. 22) ).

On the other hand, using (122), we obtain

$$
\frac{p-p_{D}}{1-\rho_{D} h_{L, D}(p)}<\frac{p-p_{D}}{1-\rho_{D} h_{R, D}(p)}, p>p_{D} .
$$

Since the function $h_{L, D}$ is strictly convex, the function $p \mapsto \frac{p-p_{D}}{1-\rho_{D} h_{L, D}(p)}$ is monotonically increasing so that

$$
\frac{\bar{p}^{*}-p_{D}}{1-\rho_{D} h_{L, D}\left(\bar{p}^{*}\right)}<\frac{p^{*}-p_{D}}{1-\rho_{D} h_{L, D}\left(p^{*}\right)}<\frac{p^{*}-p_{D}}{1-\rho_{D} h_{R, D}\left(p^{*}\right)},
$$

which contradicts the inequality (119). We have thus proved our assertion from which it follows that

$$
\bar{u}^{*}<u^{*}<\sigma_{3} \leq 0
$$

(cf. Fig. 23).

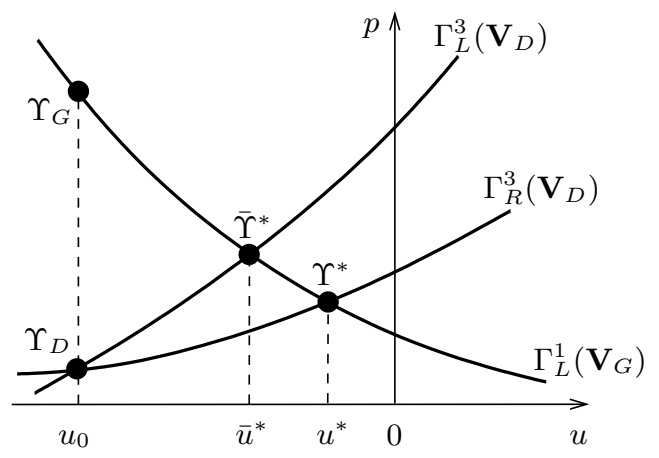

FIG. 23

Therefore it appears fairly natural to look for a solution $\mathbf{Z}=\mathbf{Z}\left(. ; \mathbf{V}_{G}, \mathbf{V}_{D}\right)$ of the coupled Riemann problem that consists of the four constant states $\mathbf{V}_{G}, \overline{\mathbf{V}}_{G}^{*}, \overline{\mathbf{V}}_{D}^{*}$ and $\mathbf{V}_{D}$ separated by a 1- $L$-rarefaction wave with end speeds $\bar{\sigma}_{1}$ and $\bar{\sigma}_{2}$, by a 2 - $L$-contact discontinuity with speed $\bar{u}^{*}<0$ and by a stationary discontinuity. This function is discontinuous at $x=0$ :

$$
\mathbf{Z}(0-)=\overline{\mathbf{V}}_{D}^{*}, \mathbf{Z}(0+)=\mathbf{V}_{D} .
$$

Again we need to check the coupling constraints. We first observe that $\overline{\mathbf{V}}_{D}^{*}$ is connected to $\mathbf{V}_{D}$ by a 3 - $L$-shock wave with speed $\bar{\sigma}_{3}>0$, which yields

$$
\overline{\mathbf{V}}_{D}^{*}=\mathbf{Z}_{L}\left(0-; \overline{\mathbf{V}}_{D}^{*}, \mathbf{V}_{D}\right) \Leftrightarrow \overline{\mathbf{V}}_{D}^{*} \in \mathcal{O}_{L}\left(\mathbf{V}_{D}\right) .
$$

It remains only to check the property

$$
\mathbf{V}_{D}=\mathbf{Z}_{R}\left(0+; \overline{\mathbf{V}}_{D}^{*}, \mathbf{V}_{D}\right) \Leftrightarrow \mathbf{V}_{D} \in \mathcal{O}_{R}\left(\overline{\mathbf{V}}_{D}^{*}\right) .
$$

We notice (cf. Fig. 24) that $\mathbf{Z}_{R}\left(. ; \overline{\mathbf{V}}_{D}^{*}, \mathbf{V}_{D}\right)$ consists of a 1-rarefaction wave, a 2-contact discontinuity and a 3 -shock wave. Then (123) holds if and only if the speed of this 3 -shock 
is nonpositive and, by the results of Section 3 if and only if the following conditions are satisfied:

$$
\begin{gathered}
u_{0} \leq-c_{R, D}, \\
u_{s}^{3} \geq \bar{u}^{*}-\Phi_{R, \overline{\mathbf{V}}_{D}^{*}}\left(p_{s}^{3}\right),
\end{gathered}
$$

where $p_{s}^{3}<p_{D}$ is the solution of the equation

$$
\rho_{D} u_{0} h_{R, D}(p)=u_{0}+\Phi_{R, D}(p)
$$

while $u_{s}^{3}$ is given by

$$
u_{s}^{3}=u_{0}+\Phi_{R, D}\left(p_{s}^{3}\right) .
$$

By the Lax entropy condition, we have

$$
u_{0}+c_{R, D}<\sigma_{3}<0
$$

so that we need only to check the condition (124). Setting

$$
\Upsilon_{s}^{3}=\left(u_{s}^{3}, p_{s}^{3}\right) \in \Gamma_{R}^{3}\left(\mathbf{V}_{D}\right),
$$

this amounts to checking that the intersection point $\overline{\bar{\Upsilon}}^{*}$ of the curves $\Gamma_{R}^{1}\left(\overline{\mathbf{V}}_{D}^{*}\right)$ and $\Gamma_{R}^{3}\left(\mathbf{V}_{D}\right)$ is located below $\Upsilon_{s}^{3}$ on $\Gamma_{R}^{3}\left(\mathbf{V}_{D}\right)$. Now, using the assumption $\mathbf{H . 3}$, we have already seen that $\Phi_{L, D}(p)<\Phi_{R, D}(p)$ for $p>p_{D}$, which means that (121) indeed holds. Hence we obtain for $p<\bar{p}^{*}$,

$$
\bar{u}^{*}-\Phi_{R, \overline{\mathbf{V}}_{D}^{*}}(p)>\bar{u}^{*}-\Phi_{L, \overline{\mathbf{V}}_{D}^{*}}(p)=u_{0}-\Phi_{L, G}(p)
$$

or equivalently that $\Gamma_{R}^{1}\left(\overline{\mathbf{V}}_{D}^{*}\right)$ is above $\Gamma_{L}^{1}\left(\mathbf{V}_{G}\right)$ for $p<\bar{p}^{*}$. The point $\bar{\Upsilon}^{*}$ is therefore located above $\Upsilon^{*}$ on the curve $\Gamma_{R}^{3}\left(\mathbf{V}_{D}\right)$. On the other hand, since $\sigma_{3}$ is $<0$, the point $\Upsilon^{*}$ is below $\Upsilon_{s}^{3}$ on $\Gamma_{R}^{3}\left(\mathbf{V}_{D}\right)$. Then, provided that the equations of state are close enough so that the curves $\Gamma_{L}^{1}\left(\mathbf{V}_{G}\right)$ and $\Gamma_{R}^{1}\left(\overline{\mathbf{V}}_{D}^{*}\right)$ are close enough, $\bar{\Upsilon}^{*}$ is located between the points $\Upsilon^{*}$ and $\Upsilon_{s}^{3}$ on the curve $\Gamma_{R}^{3}\left(\mathbf{V}_{D}\right)$ and the proposed function $\mathbf{Z}$ is indeed a solution of the coupled Riemann problem. Note that this solution corresponds to the case 6.3.a of Section 6 .
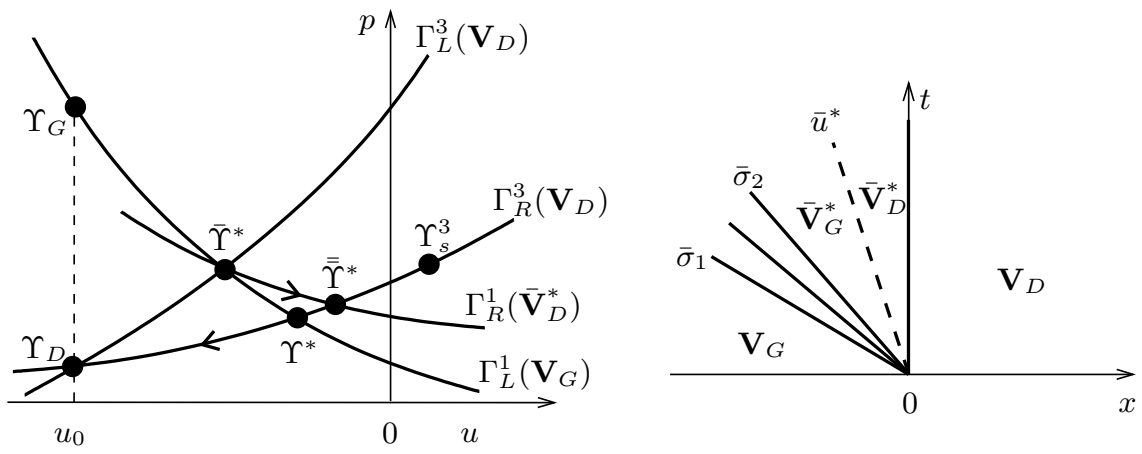

FIG. 24

However, if $\bar{\Upsilon}^{*}$ is located above $\Upsilon_{s}^{3}$ on $\Gamma_{R}^{3}\left(\mathbf{V}_{D}\right)$, the discontinuity at $x=0$ is no longer admissible: the construction of a solution of the coupled Riemann problem still remains an open question. 
8. Conclusion. We have studied in this paper a fairly general method of coupling two Euler systems provided with different equations of state. We have been able to characterize the coupling conditions and to detail all the possible solutions of the coupled Riemann problem (CRP). As an application, we have solved this CRP when $u_{G}=u_{D}$ except in one single case. However several problems still remain open concerning the existence and uniqueness of the solution of the CPR. On the one hand, we conjecture that the CPR possesses at least one global solution. A first step in this direction would consist in obtaining this existence for sufficiently close initial data $\mathbf{V}_{G}$ and $\mathbf{V}_{D}$. Recall that such an existence result of $\mathbf{V}$-continuous subsonic solutions has been obtain in Theorem 3. On the other hand, concerning uniqueness, we have observed that there exists at most a $\mathbf{V}$ discontinuous solution but that this is not true of $\mathbf{V}$-continuous solutions. Indeed, in one case, we have obtained the existence of a one-parameter continuum of solutions (cf. the case (iii) of section 4). Hence two open natural questions arise:

(i) Is it possible for a CRP to have a $\mathbf{V}$-discontinuous solution together with a $\mathbf{V}$ continuous one?

(ii) When the CRP has several solutions, does there exist a criterion for selecting the natural one in some appropriate sense?

In fact, this paper constitutes only one step in the analysis of the coupling of more general nonlinear hyperbolic systems. In particular, it remains to extend our results to complex coupling problems encountered in thermohydraulics. A natural and useful extension would be the analysis of the coupling of homogeneous equilibrium and homogeneous relaxation models as those considered in $\mathrm{ACC}^{+} 06 \mathrm{a}$ where the phase transitions liquid-vapor should be taken into account (see [Cae06] for preliminary results).

\section{REFERENCES}

$\left[\mathrm{ACC}^{+} 05 \mathrm{a}\right]$ A. Ambroso, C. Chalons, F. Coquel, E. Godlewski, F. Lagoutière, P.-A. Raviart, and N. Seguin. Coupling of multiphase flow models. In Proceedings of the Eleventh International Meeting on Nuclear Thermal-Hydraulics (NURETH), 2005.

$\left[\mathrm{ACC}^{+} 05 \mathrm{~b}\right]$ A. Ambroso, C. Chalons, F. Coquel, E. Godlewski, F. Lagoutière, P.-A. Raviart, and N. Seguin. Homogeneous models with phase transition: Coupling by finite volume methods. In Finite volumes for complex applications, IV (Marrakech, 2005), pages 483-492. Hermes Science, 2005.

$\left[\mathrm{ACC}^{+}\right.$06a] A. Ambroso, C. Chalons, F. Coquel, E. Godlewski, F. Lagoutière, P.-A. Raviart, and N. Seguin. The coupling of homogeneous models for two-phase flows. Int. J. Finite Volumes, 4(1):1-39, 2007.

$\left[\mathrm{ACC}^{+} 06 \mathrm{~b}\right]$ A. Ambroso, C. Chalons, F. Coquel, E. Godlewski, F. Lagoutière, P.-A. Raviart, and N. Seguin. Extension of interface coupling to general Lagrangian systems. In Springer, editor, Numerical Mathematics and Advanced Applications (Enumath, 2005), pages 852-860, 2006. MR2303716 (2007k:35311)

$\left[\mathrm{ACC}^{+} 06 \mathrm{c}\right]$ A. Ambroso, C. Chalons, F. Coquel, E. Godlewski, F. Lagoutière, P.-A. Raviart, and N. Seguin. Coupling of general Lagrangian systems. Mathematics of Computation, vol. 77, no. 262, pp. 909-941, 2008. MR2373185

[AK01] R. Abgrall and S. Karni. Computations of compressible multifluids. J. Comput. Phys., 169(2):594-623, 2001. MR1836526 (2002b:76077)

[BHK06a] M. K. Banda, M. Herty, and A. Klar. Coupling conditions for gas networks governed by the isothermal Euler equations. Netw. Heterog. Media, 1(2):295-314 (electronic), 2006. MR2223073 (2006m:35229)

[BHK06b] M. K. Banda, M. Herty, and A. Klar. Gas flow in pipeline networks. Netw. Heterog. Media, 1(1):41-56 (electronic), 2006. MR.2219276(2007d:76193) 
[Bou06] B. Boutin. Couplage de lois de conservation scalaires par une régularisation à la Dafermos. Master's thesis, Université Pierre et Marie Curie-Paris 6, 2006.

[BV06] F. Bachmann and J. Vovelle. Existence and uniqueness of entropy solution of scalar conservation laws with a flux function involving discontinuous coefficients. Comm. Partial Differential Equations, 31:371-395, 2006. MR2209759

[Cae06] F. Caetano. Sur certains problèmes de linéarisation et de couplage pour les systèmes hyperboliques non linéaires. Ph.D. thesis, Université Pierre et Marie Curie-Paris 6, France, 2006.

[CG06] R. M. Colombo and M. Garavello. A well posed Riemann problem for the $p$-system at a junction. Netw. Heterog. Media, 1(3):495-511 (electronic), 2006. MR2247787 (2007j:35125)

[GL96] J. M. Greenberg and A. Y. Leroux. A well-balanced scheme for the numerical processing of source terms in hyperbolic equations. SIAM J. Numer. Anal., 33(1):1-16, 1996. MR1377240 (97c:65144)

[GL04] P. Goatin and P. G. LeFloch. The Riemann problem for a class of resonant hyperbolic systems of balance laws. Ann. Inst. H. Poincaré Anal. Non Linéaire, 21(6):881-902, 2004. MR2097035 (2006i:35225)

[GLTR05] E. Godlewski, K.-C. Le Thanh, and P.-A. Raviart. The numerical interface coupling of nonlinear hyperbolic systems of conservation laws. II. The case of systems. M2AN Math. Model. Numer. Anal., 39(4):649-692, 2005. MR2165674 (2006h:65133)

[GP06a] M. Garavello and B. Piccoli. Traffic flow on networks. AIMS book series, 2006. MR2328174

[GP06b] M. Garavello and B. Piccoli. Traffic flow on a road network using the Aw-Rascle model. Comm. Partial Differential Equations, 31(1-3):243-275, 2006. MR2209753 (2006k:90022)

[GR04] E. Godlewski and P.-A. Raviart. The numerical interface coupling of nonlinear hyperbolic systems of conservation laws. I. The scalar case. Numer. Math., 97(1):81-130, 2004. MR2045460 (2005e:65130)

[HR06] M. Herty and M. Rascle. Coupling conditions for a class of second-order models for traffic flow. SIAM J. Math. Anal., 38(2):595-616 (electronic), 2006. MR2237163 (2007e:35186)

[Hur06] O. Hurisse. Techniques de couplage de modèles hyperboliques en thermohydraulique diphasique. Ph.D. thesis, Université de Provence, France, 2006.

[IT92] E. Isaacson and B. Temple. Nonlinear resonance in systems of conservation laws. SIAM J. Appl. Math., 52(5):1260-1278, 1992. MR1182123 (93f:35140)

[IT95] E. Isaacson and B. Temple. Convergence of the $2 \times 2$ Godunov method for a general resonant nonlinear balance law. SIAM J. Appl. Math., 55(3):625-640, 1995. MR 1331577(96c:65146)

[KR95] C. Klingenberg and N. H. Risebro. Convex conservation laws with discontinuous coefficients. Existence, uniqueness and asymptotic behavior. Comm. Partial Differential Equations, 20(11-12):1959-1990, 1995. MR1361727(96i:35082) 